\title{
New Fe II energy levels from stellar spectra ${ }^{\star}$
}

\author{
F. Castelli ${ }^{1}$ and R. L. Kurucz ${ }^{2}$ \\ 1 Istituto Nazionale di Astrofisica - Osservatorio Astronomico di Trieste, via Tiepolo 11, 34131 Trieste, Italy \\ e-mail: castelli@oats.inaf.it \\ 2 Harvard-Smithsonian Center for Astrophysics, 60 Garden Street, Cambridge, MA 02138, USA
}

Received 1 June 2010 / Accepted 29 June 2010

ABSTRACT

\begin{abstract}
Aims. The spectra of B-type and early A-type stars show numerous unidentified lines in the whole optical range, especially in the 5100-5400 Å interval. Because Fe II transitions to high energy levels should be observed in this region, we used semiempirical predicted wavelengths and gf-values of Fe II to identify unknown lines.

Methods. Semiempirical line data for Fe II computed by Kurucz are used to synthesize the spectrum of the slow-rotating, Fe -overabundant CP star HR 6000.

Results. We determined a total of 109 new $4 \mathrm{f}$ levels for Fe II with energies ranging from $122324 \mathrm{~cm}^{-1}$ to $128110 \mathrm{~cm}^{-1}$. They belong to the Fe II subconfigurations $3 d^{6}\left({ }^{3} \mathrm{P}\right) 4 \mathrm{f}$ (10 levels), $3 \mathrm{~d}^{6}\left({ }^{3} \mathrm{H}\right) 4 \mathrm{f}$ (36 levels), $3 \mathrm{~d}^{6}\left({ }^{3} \mathrm{~F}\right) 4 \mathrm{f}$ (37 levels), and $3 \mathrm{~d}^{6}\left({ }^{3} \mathrm{G}\right) 4 \mathrm{f}(26$ levels). We also found 14 even levels from $4 \mathrm{~d}$ ( 3 levels), $5 \mathrm{~d}$ (7 levels), and $6 \mathrm{~d}$ (4 levels) configurations. The new levels have allowed us to identify more than $50 \%$ of the previously unidentified lines of HR 6000 in the wavelength region $3800-8000 \AA$. Tables listing the new energy levels are given in the paper; tables listing the spectral lines with $\log g f \geq-1.5$ that are transitions to the $4 \mathrm{f}$ energy levels are given in the Online Material. These new levels produce 18000 lines throughout the spectrum from the ultraviolet to the infrared.
\end{abstract}

Key words. line: identification - atomic data - stars: atmospheres - stars: chemically peculiar - stars: individual: HR 6000

\section{Introduction}

In a previous paper (Castelli et al. 2009) (Paper I) we have determined 21 new $3 d^{6}\left({ }^{3} \mathrm{H}\right) 4 \mathrm{f}$ high energy levels of Fe II on the basis of predicted energy levels, computed log $g f$ values for Fe II, and unidentified lines in UVES high resolution, high signal-to-noise spectra of HR 6000 and 46 Aql. Both stars are iron overabundant CP stars and have rotational velocity $v \sin i$ of the order of $1.5 \mathrm{~km} \mathrm{~s}^{-1}$ and $1.0 \mathrm{~km} \mathrm{~s}^{-1}$, respectively.

In this paper we continue the effort to determine new highenergy levels of Fe II. We used the same spectra and models for HR 6000 that we adopted in Paper I, together with Fe II line lists which include transitions between observed-observed, observedpredicted, and predicted-predicted energy levels. In this paper we increase the number of the new energy levels from the 21 listed in Paper I, to a total of 109 energy levels, which belong to the Fe II subconfigutations: $3 \mathrm{~d}^{6}\left({ }^{3} \mathrm{P}\right) 4 \mathrm{f}(10$ levels $), 3 \mathrm{~d}^{6}\left({ }^{3} \mathrm{H}\right) 4 \mathrm{f}$ (36 levels), $3 d^{6}\left({ }^{3} \mathrm{~F}\right) 4 \mathrm{f}$ (37 levels), and $3 d^{6}\left({ }^{3} \mathrm{G}\right) 4 \mathrm{f}$ (26 levels), and 14 levels from the even configurations $4 \mathrm{~d}$ (3 levels), $5 \mathrm{~d}$ (7 levels), and $6 \mathrm{~d}$ (4 levels). The new levels have allowed us to identify more than the $50 \%$ of the previously unidentified lines in the wavelength region 3800-8000 $\AA$ of HR 6000 (Castelli \& Hubrig 2007). The method that we adopted to determine the new energy levels is the same as described in Paper I. It is recalled here in Sect. 3. The comparison of the observed spectrum of HR 6000 with the synthetic spectrum which includes the new Fe II lines is available on the Castelli web site ${ }^{1}$.

\footnotetext{
* Tables 6-9 are also available in electronic form at the CDS via anonymous ftp to cdsarc.u-strasbg.fr (130.79.128.5) or via http://cdsarc.u-strasbg.fr/viz-bin/qcat?J/A+A/520/A57

1 http://wwwuser.oat.ts.astro.it/castelli/hr6000new/ hr6000.html
}

\section{The star HR 6000}

According to Paper I, the CP star HR 6000 (HD 144667) has an estimated rotational velocity of $1.5 \mathrm{~km} \mathrm{~s}^{-1}$. The model stellar parameters for an individual abundance ATLAS12 (Kurucz 2005) model are $T_{\text {eff }}=13450 \mathrm{~K}, \log g=4.3$. In addition to the large iron overabundance $[+0.9]$, overabundances of $\mathrm{Xe}([+4.6]), \mathrm{P}(>[+1.5]), \mathrm{Ti}([+0.55]), \mathrm{Cr}([+0.2]), \mathrm{Mn}([+1.5])$, $\mathrm{Y}([+1.2])$, and $\mathrm{Hg}([+2.7])$ were observed. This peculiar chemical composition, together with the underabundances of $\mathrm{He}, \mathrm{C}, \mathrm{N}$, $\mathrm{O}, \mathrm{Al}, \mathrm{Mg}, \mathrm{Si}, \mathrm{S}, \mathrm{Cl}, \mathrm{Sc}, \mathrm{V}, \mathrm{Co}, \mathrm{Ni}$, and $\mathrm{Sr}$ gives rise to an optical line spectrum very rich in Fe II lines, with transitions involving upper energy levels close to the ionization limit (Johansson 2009). Also numerous Fe I and Fe III lines are observable in the spectrum.

\section{The method}

To determine the new energy levels we used high-resolution UVES spectra of HR 6000 (see PaperI), the corresponding synthetic spectrum, and the list of the computed transitions with predicted values for levels with no experimentally available energies. Predicted energy levels and $\log g f$ values were computed by Kurucz with his version of the Cowan (1981) code (Kurucz 2009). The calculation included 46 even configurations $d^{7}, d^{6} 4 s-9 s, d^{6} 4 d-9 d, d^{6} 5 g-9 g, d^{6} 7 i-9 i$, $d^{6} 91, d^{5} 4 s^{2}, d^{5} 4 s 5 s-9 s, d^{5} 4 s 4 d-9 d, d^{5} 4 s 5 g-9 g, d^{5} 4 s 7 i-9 i$, $d^{5} 4 s 91, d^{4} 4 s^{2} 4 d$, and $d^{5} 4 p^{2}$ with 19771 levels least-squares fitted to 418 known levels. The 39 odd configurations included $d^{6} 4 p-9 p, d^{6} 4 f-9 f, d^{6} 6 h-9 h, d^{6} 8 k-9 k, d^{5} 4 s 4 p-9 p$, $d^{5} 4 s 4 f-9 f, d^{5} 4 s 6 h-9 h, d^{5} 4 s 8 k-9 k, d^{4} 4 s^{2} 4 p-5 p$, and $d^{4} 4 s^{2} 4 f$ with 19652 levels least-squares fitted to 596 known levels. The calculations were done in LS coupling with all configuration interactions included, with scaled Hartree-Fock starting 
Table 1. Fe II energy levels for the $3 d^{6}\left({ }^{3} \mathrm{P}\right) 4 \mathrm{f}$ subconfiguration.

\begin{tabular}{|c|c|c|c|c|c|c|c|c|c|c|c|}
\hline $\begin{array}{l}\text { Design- } \\
\text { ation }\end{array}$ & $J$ & $\begin{array}{l}\text { Energy } \\
\mathrm{cm}^{-1}\end{array}$ & $\begin{array}{c}\mathrm{c}-\mathrm{o} \\
\mathrm{cm}^{-1}\end{array}$ & $\begin{array}{l}\text { Design- } \\
\text { ation }\end{array}$ & $J$ & $\begin{array}{l}\text { Energy } \\
\mathrm{cm}^{-1}\end{array}$ & $\begin{array}{l}\mathrm{c}-\mathrm{O} \\
\mathrm{cm}^{-1}\end{array}$ & $\begin{array}{l}\text { Design- } \\
\text { ation }\end{array}$ & $J$ & $\begin{array}{l}\text { Energy } \\
\mathrm{cm}^{-1}\end{array}$ & $\begin{array}{l}\mathrm{c}-\mathrm{O} \\
\mathrm{cm}^{-1}\end{array}$ \\
\hline $2[5]$ & $\begin{array}{r}11 / 2 \\
9 / 2\end{array}$ & $\begin{array}{l}122351.810 \\
122324.142\end{array}$ & $\begin{array}{l}-20.236 \\
-18.980\end{array}$ & & & & & & & & \\
\hline $2[4]$ & $\begin{array}{l}9 / 2 \\
7 / 2\end{array}$ & $\begin{array}{l}122355.116 \\
122355.553\end{array}$ & $\begin{array}{l}-6.685 \\
-6.801\end{array}$ & $1[4]$ & $\begin{array}{l}9 / 2 \\
7 / 2\end{array}$ & $\begin{array}{l}123629.520 \\
123637.833\end{array}$ & $\begin{array}{l}-4.606 \\
-6.417\end{array}$ & & & & \\
\hline $2[3]$ & $\begin{array}{l}7 / 2 \\
5 / 2\end{array}$ & $\begin{array}{c}122351.488 \\
(122353.541)\end{array}$ & -18.489 & $1[3]$ & $\begin{array}{l}7 / 2 \\
5 / 2\end{array}$ & $\begin{array}{l}123615.875 \\
123649.493\end{array}$ & $\begin{array}{l}-2.642 \\
-5.687\end{array}$ & $0[3]$ & $\begin{array}{l}7 / 2 \\
5 / 2\end{array}$ & $\begin{array}{c}(124167.229) \\
124157.060\end{array}$ & +15.841 \\
\hline $2[2]$ & $\begin{array}{l}5 / 2 \\
3 / 2\end{array}$ & $\begin{array}{l}(122342.921) \\
(122336.098)\end{array}$ & & $1[2]$ & $\begin{array}{l}5 / 2 \\
3 / 2\end{array}$ & $\begin{array}{l}(123637.063) \\
(123646.360)\end{array}$ & & & & & \\
\hline $2[1]$ & $\begin{array}{l}3 / 2 \\
1 / 2 \\
\end{array}$ & $\begin{array}{l}(122358.405) \\
(122332.608) \\
\end{array}$ & & & & & & & & & \\
\hline
\end{tabular}

Notes. Energies between parentheses are predicted values.

guesses, and with Hartree-Fock transition integrals. A total of 7080169 lines were saved from the transition array of which 102833 lines are between known levels and have good wavelengths.

The computed line list was sorted into tables of all the strong lines connected to every predicted level. When a given predicted level gives rise to at least two Fe II lines having $\log g f \geq-1.0$, we selected one of these transitions and searched in the spectrum for those unidentified lines which have wavelength within $\pm 50 \AA$ and residual flux within about $\pm 5 \%$ of those of the selected predicted line. From the observed wavelength of one of these unidentified lines and from the known energy of the lower or upper level of the predicted transition, we derived a possible energy for the predicted level. If most of transitions obtained with this energy correspond to lines observed in the spectrum, we kept the tentative energy value as a real value, otherwise we repeated the procedure using another line taken from the unidentified ones, and continued the searching until we found that energy for which most of the predicted lines correspond to the observed lines. Whenever one or more new levels were found, the whole semiempirical calculation was repeated to produce improved predicted wavelengths and $\log g f$-values. Because all configuration interactions were included, and because the mixing is exceptionally strong in the $4 \mathrm{~d}$ and $5 \mathrm{~d}$ configurations, every new level changed the predictions. Mixing between close levels can produce large uncertainties in the $\log g f$ values for lines that involve those levels.

This procedure is very successfull for levels which produce two or more transitions with $\log g f>0.0$, but becomes more and more difficult as the intensity of the predicted lines decreases. In fact, weak lines are usually blended with stronger components, so that the method may fail in these cases.

\section{The new energy levels}

The new energy levels of the $3 d^{6}\left({ }^{3} \mathrm{P}\right) 4 \mathrm{f}, 3 \mathrm{~d}^{6}\left({ }^{3} \mathrm{H}\right) 4 \mathrm{f}, 3 \mathrm{~d}^{6}\left({ }^{3} \mathrm{~F}\right) 4 \mathrm{f}$, and $3 \mathrm{~d}^{6}\left({ }^{3} \mathrm{G}\right) 4 \mathrm{f}$ subconfigurations and from the even configurations $3 d^{6} 4 d, 3 d^{6} 5 d$, and $3 d^{6} 6 d$ are listed in Tables $1-5$. Because the $3 \mathrm{~d}^{6} 4 \mathrm{f}$ states of Fe II tend to appear in pairs we have used the $j_{\mathrm{c}}[K]_{j}$ notation of $j K$ coupling for them, where $j_{\mathrm{c}}$ is the total angular momentum of the core and $\boldsymbol{K}=\boldsymbol{j}_{\mathrm{c}}+\boldsymbol{l}$ is the coupling of $\boldsymbol{j}_{\mathrm{c}}$ with the orbital angular momentum $\boldsymbol{l}$ of the active electron. The level pairs correspond to the two separate values of the total angular momentum $\boldsymbol{J}$ obtained when the spin $\boldsymbol{s}= \pm 1 / 2$ of the active electron is added to $\boldsymbol{K}$. The positive energies are those obtained by comparing observed and predicted line profiles, as described in Sect. 3 and shown in Fig. 2. The energies between parentheses in Tables 1-4 are predicted values for which we have been not able to find the corresponding observed level. The reason for the failure is that either all the lines from the energy level are weak or, even if some of the transitions are predicted as moderately strong $(\log g f>0.0)$, they are blended with other stronger components, so that their identification is uncertain. The columns with label "c-o" in Tables $1-5$ show the difference between the predicted and observed energy levels.

The $4 \mathrm{~d}$ even energy levels listed in Table 5 give rise to some of the transitions listed in the Online Material. The strongest transitions related with the $5 \mathrm{~d}$, and $6 \mathrm{~d}$ even energy levels occur in the $6000-8000 \AA$ region and in the $4000-5000 \AA$ region, respectively. The transitions to the odd energy levels are discussed in Sect. 5

The observed energy levels, the least squares fits, the predicted energy levels, and the line lists can be found on the Kurucz web site ${ }^{2}$. The observed levels come from the following sources: Johansson (1978), Sugar \& Corliss (1985), Adam et al. (1987), Johansson \& Baschek (1988), Johansson (1988, private comm.), Rosberg \& Johansson (1992), Castelli et al. (2008), Castelli et al. (2009), and this work. The calculations on the web site are updated whenever there are improvements to the energy levels.

\section{The new Fe II lines}

The new Fe II lines in the $3800-8000 \AA$ region, produced by transitions to the Fe II subconfigurations $\left({ }^{3} \mathrm{P}\right) 4 \mathrm{f},\left({ }^{3} \mathrm{H}\right) 4 \mathrm{f},\left({ }^{3} \mathrm{~F}\right) 4 \mathrm{f}$, and $\left({ }^{3} \mathrm{G}\right) 4 \mathrm{f}$, are shown in Tables $6-9$, respectively. Only lines with $\log g f \geq-1.50$ are listed, because lines with lower $\log g f$ values are not observable in this wavelength region of HR 6000. The new Fe II lines are mostly concentrated in the 5100-5400 interval. The upper energy levels (Cols. 1-4) were derived as described in Sect. 3, the lower energy levels (Cols.5-6) are those described in Sect.4, the calculated wavelength (Col.7) is the Ritz wavelength in air, the $\log g f$ values (Col. 8) were computed by Kurucz, the observed wavelengths (Col.9) are the wavelengths of lines well observable in the HR 6000 spectrum.

${ }^{2}$ http://kurucz.harvard.edu/atoms/2601 
F. Castelli and R. L. Kurucz: New Fe II energy levels from stellar spectra

Table 2. Fe II energy levels for the $3 d^{6}\left({ }^{3} \mathrm{H}\right) 4 \mathrm{f}$ subconfiguration.

\begin{tabular}{|c|c|c|c|c|c|c|c|c|c|c|c|}
\hline $\begin{array}{l}\text { Design- } \\
\text { ation }\end{array}$ & $J$ & $\begin{array}{l}\text { Energy } \\
\mathrm{cm}^{-1}\end{array}$ & $\begin{array}{l}\mathrm{c}-\mathrm{o} \\
\mathrm{cm}^{-1}\end{array}$ & $\begin{array}{l}\text { Design- } \\
\text { ation }\end{array}$ & $J$ & $\begin{array}{l}\text { Energy } \\
\mathrm{cm}^{-1}\end{array}$ & $\begin{array}{l}\mathrm{c}-\mathrm{O} \\
\mathrm{cm}^{-1}\end{array}$ & $\begin{array}{l}\text { Design- } \\
\text { ation }\end{array}$ & $J$ & $\begin{array}{l}\text { Energy } \\
\mathrm{cm}^{-1}\end{array}$ & $\begin{array}{l}\mathrm{c}-\mathrm{o} \\
\mathrm{cm}^{-1}\end{array}$ \\
\hline $6[9]$ & $\begin{array}{l}19 / 2 \\
17 / 2\end{array}$ & $\begin{array}{l}122954.180 \\
122952.730\end{array}$ & $\begin{array}{l}+14.465 \\
+20.251\end{array}$ & & & & & & & & \\
\hline $6[8]$ & $\begin{array}{l}17 / 2 \\
15 / 2\end{array}$ & $\begin{array}{l}123007.910 \\
122910.920\end{array}$ & $\begin{array}{l}+26.752 \\
-16.531\end{array}$ & $5[8]$ & $\begin{array}{l}17 / 2 \\
15 / 2\end{array}$ & $\begin{array}{l}123219.200 \\
123193.090\end{array}$ & $\begin{array}{l}-10.198 \\
-17.864\end{array}$ & & & & \\
\hline $6[7]$ & $\begin{array}{l}15 / 2 \\
13 / 2\end{array}$ & $\begin{array}{l}123018.430 \\
123015.400\end{array}$ & $\begin{array}{l}+34.439 \\
+40.333\end{array}$ & $5[7]$ & $\begin{array}{l}15 / 2 \\
13 / 2\end{array}$ & $\begin{array}{l}123238.440 \\
123168.680\end{array}$ & $\begin{array}{r}-6.653 \\
-33.645\end{array}$ & $4[7]$ & $\begin{array}{l}15 / 2 \\
13 / 2\end{array}$ & $\begin{array}{l}123396.250 \\
123355.490\end{array}$ & $\begin{array}{l}-33.027 \\
-36.436\end{array}$ \\
\hline $6[6]$ & $\begin{array}{l}13 / 2 \\
11 / 2\end{array}$ & $\begin{array}{l}122990.620 \\
123037.430\end{array}$ & $\begin{array}{r}-2.720 \\
+26.878\end{array}$ & $5[6]$ & $\begin{array}{l}13 / 2 \\
11 / 2\end{array}$ & $\begin{array}{l}123249.650 \\
123270.340\end{array}$ & $\begin{array}{l}-6.519 \\
+0.899\end{array}$ & $4[6]$ & $\begin{array}{l}13 / 2 \\
11 / 2\end{array}$ & $\begin{array}{l}123414.730 \\
123427.119\end{array}$ & $\begin{array}{l}-32.244 \\
-33.418\end{array}$ \\
\hline $6[5]$ & $\begin{array}{r}11 / 2 \\
9 / 2\end{array}$ & $\begin{array}{l}123002.288 \\
123026.350\end{array}$ & $\begin{array}{l}+33.455 \\
+18.587\end{array}$ & $5[5]$ & $\begin{array}{r}11 / 2 \\
9 / 2\end{array}$ & $\begin{array}{l}123251.470 \\
123269.378\end{array}$ & $\begin{array}{l}-1.320 \\
+2.937\end{array}$ & $4[5]$ & $\begin{array}{r}11 / 2 \\
9 / 2\end{array}$ & $\begin{array}{l}123441.100 \\
123435.468\end{array}$ & $\begin{array}{l}-26.889 \\
-17.705\end{array}$ \\
\hline $6[4]$ & $\begin{array}{l}9 / 2 \\
7 / 2\end{array}$ & $\begin{array}{l}122988.215 \\
122980.408\end{array}$ & $\begin{array}{l}+30.836 \\
+26.752\end{array}$ & $5[4]$ & $\begin{array}{l}9 / 2 \\
7 / 2\end{array}$ & $\begin{array}{l}123258.994 \\
123258.021\end{array}$ & $\begin{array}{l}-1.556 \\
-1.362\end{array}$ & $4[4]$ & $\begin{array}{l}9 / 2 \\
7 / 2\end{array}$ & $\begin{array}{l}123460.690 \\
123435.277\end{array}$ & $\begin{array}{l}-26.898 \\
-16.103\end{array}$ \\
\hline $6[3]$ & $\begin{array}{l}7 / 2 \\
5 / 2\end{array}$ & $\begin{array}{c}122946.419 \\
(122967.896)\end{array}$ & +21.403 & $5[3]$ & $\begin{array}{l}7 / 2 \\
5 / 2\end{array}$ & $\begin{array}{c}123235.165 \\
(123248.017)\end{array}$ & +3.471 & $4[3]$ & $\begin{array}{l}7 / 2 \\
5 / 2\end{array}$ & $\begin{array}{l}123451.449 \\
123430.181\end{array}$ & $\begin{array}{l}-21.115 \\
-16.906\end{array}$ \\
\hline & & & & $5[2]$ & $\begin{array}{l}5 / 2 \\
3 / 2\end{array}$ & $\begin{array}{l}123211.159 \\
123213.323\end{array}$ & $\begin{array}{r}-1.017 \\
-12.585\end{array}$ & $4[2]$ & $\begin{array}{l}5 / 2 \\
3 / 2\end{array}$ & $\begin{array}{l}(123401.927) \\
(123384.857)\end{array}$ & \\
\hline & & & & & & & & $4[1]$ & $\begin{array}{l}3 / 2 \\
1 / 2\end{array}$ & $\begin{array}{l}(123356.410) \\
(123343.705)\end{array}$ & \\
\hline
\end{tabular}

Notes. Energies between parentheses are predicted values.

Table 3. Fe II energy levels for the $3 d^{6}\left({ }^{3} F\right) 4 f$ subconfiguration. Energies between parentheses are predicted values.

\begin{tabular}{|c|c|c|c|c|c|c|c|c|c|c|c|}
\hline $\begin{array}{l}\text { Design- } \\
\text { ation }\end{array}$ & $J$ & $\begin{array}{l}\text { Energy } \\
\mathrm{cm}^{-1}\end{array}$ & $\begin{array}{l}\mathrm{c}-\mathrm{o} \\
\mathrm{cm}^{-1}\end{array}$ & $\begin{array}{l}\text { Design- } \\
\text { ation }\end{array}$ & $J$ & $\begin{array}{l}\text { Energy } \\
\mathrm{cm}^{-1}\end{array}$ & $\begin{array}{l}\mathrm{c}-\mathrm{o} \\
\mathrm{cm}^{-1}\end{array}$ & $\begin{array}{l}\text { Design- } \\
\text { ation }\end{array}$ & $J$ & $\begin{array}{l}\text { Energy } \\
\mathrm{cm}^{-1}\end{array}$ & $\begin{array}{l}\mathrm{c}-\mathrm{o} \\
\mathrm{cm}^{-1}\end{array}$ \\
\hline \multirow[t]{2}{*}{$4[7]$} & $15 / 2$ & 124421.468 & +12.238 & & & & & & & & \\
\hline & $13 / 2$ & 124436.436 & +36.895 & & & & & & & & \\
\hline \multirow[t]{2}{*}{$4[6]$} & $13 / 2$ & 124400.107 & +4.567 & $3[6]$ & $13 / 2$ & 124661.274 & +15.827 & & & & \\
\hline & $11 / 2$ & 124402.557 & -3.593 & & $11 / 2$ & 124656.535 & +7.092 & & & & \\
\hline \multirow[t]{2}{*}{$4[5]$} & $11 / 2$ & 124388.840 & +3.174 & $3[5]$ & $11 / 2$ & 124626.900 & +3.179 & $2[5]$ & $11 / 2$ & 124803.873 & +20.054 \\
\hline & $9 / 2$ & 124385.706 & +2.938 & & $9 / 2$ & 124636.116 & +3.120 & & $9 / 2$ & 124809.727 & +15.721 \\
\hline \multirow[t]{2}{*}{$4[4]$} & $9 / 2$ & 124401.939 & +4.674 & $3[4]$ & $9 / 2$ & 124623.120 & +3.085 & $2[4]$ & $9 / 2$ & 124793.905 & +12.624 \\
\hline & $7 / 2$ & 124385.010 & +0.698 & & $7 / 2$ & 124620.914 & +7.289 & & $7 / 2$ & 124783.748 & +15.272 \\
\hline \multirow[t]{2}{*}{$4[3]$} & $7 / 2$ & 124416.110 & +13.187 & $3[3]$ & $7 / 2$ & 124641.989 & +9.092 & $2[3]$ & $7 / 2$ & (124 814.025) & \\
\hline & $5 / 2$ & 124403.474 & +1.243 & & $5 / 2$ & 124653.022 & -8.651 & & $5 / 2$ & (124 808.178) & \\
\hline \multirow[t]{2}{*}{$4[2]$} & $5 / 2$ & 124434.563 & +23.142 & $3[2]$ & $5 / 2$ & $(124670.316)$ & & $2[2]$ & $5 / 2$ & $(124835.676)$ & \\
\hline & $3 / 2$ & 124460.410 & -11.802 & & $3 / 2$ & $(124678.325)$ & & & $3 / 2$ & (124 833.418) & \\
\hline \multirow[t]{3}{*}{$4[1]$} & $3 / 2$ & (124 487.989) & & $3[1]$ & $3 / 2$ & (124 697.077) & & $2[1]$ & $3 / 2$ & $(124876.972)$ & \\
\hline & $1 / 2$ & (124 484.721) & & & $1 / 2$ & (124 708.453) & & & $1 / 2$ & (124 874.375) & \\
\hline & & & & $3[0]$ & $1 / 2$ & 124731.762 & -4.875 & & & & \\
\hline
\end{tabular}

Notes. Energies between parentheses are predicted values.

Most of them were listed as unidentified lines in Castelli \& Hubrig $(2007)^{3}$. In the last column, comments derived from the comparison of the observed and computed spectra are added for

\footnotetext{
${ }^{3}$ http://wwwuser.oat.ts.astro.it/castelli/hr6000/ unidentified.txt
}

most lines. In a few cases, both computed and observed stellar lines correspond to lines measured by Johansson in laboratory works (Johansson 1978; Castelli et al. 2008). The notes "J78" and "lab" are added for these lines. When lines are computed weaker than the observed ones the disagreement can be due 
A\&A 520, A57 (2010)

Table 4. Fe II energy levels for the $3 d^{6}\left({ }^{3} \mathrm{G}\right) 4 \mathrm{f}$ subconfiguration. Energies between parentheses are predicted values.

\begin{tabular}{|c|c|c|c|c|c|c|c|c|c|c|c|}
\hline $\begin{array}{l}\text { Design- } \\
\text { ation }\end{array}$ & $J$ & $\begin{array}{l}\text { Energy } \\
\mathrm{cm}^{-1}\end{array}$ & $\begin{array}{l}\mathrm{c}-\mathrm{o} \\
\mathrm{cm}^{-1}\end{array}$ & $\begin{array}{l}\text { Design- } \\
\text { ation }\end{array}$ & $J$ & $\begin{array}{l}\text { Energy } \\
\mathrm{cm}^{-1}\end{array}$ & $\begin{array}{l}\mathrm{c}-\mathrm{O} \\
\mathrm{cm}^{-1}\end{array}$ & $\begin{array}{l}\text { Design- } \\
\text { ation }\end{array}$ & $J$ & $\begin{array}{l}\text { Energy } \\
\mathrm{cm}^{-1}\end{array}$ & $\begin{array}{l}\mathrm{c}-\mathrm{o} \\
\mathrm{cm}^{-1}\end{array}$ \\
\hline $5[8]$ & $\begin{array}{l}17 / 2 \\
15 / 2\end{array}$ & $\begin{array}{c}127507.241 \\
127524.1227\end{array}$ & $\begin{array}{r}-5.657 \\
+14.501\end{array}$ & & & & & & & & \\
\hline $5[7]$ & $\begin{array}{l}15 / 2 \\
13 / 2\end{array}$ & $\begin{array}{l}127484.653 \\
127515.235\end{array}$ & $\begin{array}{l}-1.445 \\
+2.816\end{array}$ & $4[7]$ & $\begin{array}{l}15 / 2 \\
13 / 2\end{array}$ & $\begin{array}{l}127892.981 \\
127895.260\end{array}$ & $\begin{array}{l}+4.313 \\
+3.367\end{array}$ & & & & \\
\hline $5[6]$ & $\begin{array}{l}13 / 2 \\
11 / 2\end{array}$ & $\begin{array}{l}127489.429 \\
127489.977\end{array}$ & $\begin{array}{l}-4.823 \\
-0.294\end{array}$ & $4[6]$ & $\begin{array}{l}13 / 2 \\
11 / 2\end{array}$ & $\begin{array}{l}127875.000 \\
127880.436\end{array}$ & $\begin{array}{l}+2.236 \\
+1.216\end{array}$ & $3[6]$ & $\begin{array}{l}13 / 2 \\
11 / 2\end{array}$ & $\begin{array}{c}128110.214 \\
(128076.012)\end{array}$ & -2.182 \\
\hline $5[5]$ & $\begin{array}{r}11 / 2 \\
9 / 2\end{array}$ & $\begin{array}{c}127482.748 \\
(127484.561)\end{array}$ & +3.147 & $4[5]$ & $\begin{array}{r}11 / 2 \\
9 / 2\end{array}$ & $\begin{array}{l}127869.158 \\
127855.952\end{array}$ & $\begin{array}{r}+0.993 \\
-16.898\end{array}$ & $3[5]$ & $\begin{array}{r}11 / 2 \\
9 / 2\end{array}$ & $\begin{array}{l}128071.171 \\
128055.658\end{array}$ & $\begin{array}{l}-10.517 \\
-16.898\end{array}$ \\
\hline $5[4]$ & $\begin{array}{l}9 / 2 \\
7 / 2\end{array}$ & $\begin{array}{l}127485.362 \\
127485.699\end{array}$ & $\begin{array}{r}-15.194 \\
+9.404\end{array}$ & $4[4]$ & $\begin{array}{l}9 / 2 \\
7 / 2\end{array}$ & $\begin{array}{c}127869.892 \\
(127871.098)\end{array}$ & -4.920 & $3[4]$ & $\begin{array}{l}9 / 2 \\
7 / 2\end{array}$ & $\begin{array}{l}128062.710 \\
128066.823\end{array}$ & $\begin{array}{l}-15.669 \\
-22.228\end{array}$ \\
\hline $5[3]$ & $\begin{array}{l}7 / 2 \\
5 / 2\end{array}$ & $\begin{array}{c}(127476.624) \\
127510.913\end{array}$ & +9.552 & $4[3]$ & $\begin{array}{l}7 / 2 \\
5 / 2\end{array}$ & $\begin{array}{c}(127877.776) \\
127874.745\end{array}$ & +5.549 & $3[3]$ & $\begin{array}{l}7 / 2 \\
5 / 2\end{array}$ & $\begin{array}{c}(128047.849) \\
128063.103\end{array}$ & -8.192 \\
\hline $5[2]$ & $\begin{array}{l}5 / 2 \\
3 / 2\end{array}$ & $\begin{array}{c}(127499.343) \\
127487.681\end{array}$ & -0.341 & $4[2]$ & $\begin{array}{l}5 / 2 \\
3 / 2\end{array}$ & $\begin{array}{l}(127868.807) \\
(127895.930)\end{array}$ & & $3[2]$ & $\begin{array}{l}5 / 2 \\
3 / 2\end{array}$ & $\begin{array}{c}128089.313 \\
(128069.044)\end{array}$ & +10.032 \\
\hline & & & & $4[1]$ & $\begin{array}{l}3 / 2 \\
1 / 2\end{array}$ & $\begin{array}{l}(127876.787) \\
(127898.510)\end{array}$ & & $3[1]$ & $\begin{array}{l}3 / 2 \\
1 / 2\end{array}$ & $\begin{array}{l}(128099.051) \\
(128099.237)\end{array}$ & \\
\hline & & & & & & & & $3[0]$ & $1 / 2$ & (128 161.312) & \\
\hline
\end{tabular}

Notes. Energies between parentheses are predicted values.

Table 5. Fe II new levels from $3 d^{6} 4 d, 3 d^{6} 5 d$, and $3 d^{6} 6 d$ configurations.

\begin{tabular}{ccccc}
\hline \hline \multicolumn{2}{c}{ Designation } & $J$ & $\begin{array}{c}\text { Energy } \\
\mathrm{cm}^{-1}\end{array}$ & $\begin{array}{c}\mathrm{c}-\mathrm{o} \\
\mathrm{cm}^{-1}\end{array}$ \\
\hline $3 \mathrm{~d}^{6}\left({ }^{3} \mathrm{P}\right) 4 \mathrm{~d}$ & ${ }^{2} \mathrm{~F}$ & $7 / 2$ & 103191.917 & +27.014 \\
$3 \mathrm{~d}^{6}\left({ }^{3} \mathrm{P}\right) 4 \mathrm{~d}$ & ${ }^{2} \mathrm{D}$ & $5 / 2$ & 103597.402 & -5.701 \\
$3 \mathrm{~d}^{6}\left({ }^{3} \mathrm{~F}\right) 4 \mathrm{~d}$ & ${ }^{2} \mathrm{~F}$ & $7 / 2$ & 105775.491 & -42.697 \\
& & & & \\
$3 \mathrm{~d}^{6}\left({ }^{3} \mathrm{H}\right) 5 \mathrm{~d}$ & ${ }^{4} \mathrm{H}$ & $13 / 2$ & 124208.725 & +47.495 \\
$3 \mathrm{~d}^{6}\left({ }^{3} \mathrm{H}\right) 5 \mathrm{~d}$ & ${ }^{4} \mathrm{G}$ & $11 / 2$ & 124251.805 & +44.041 \\
$3 \mathrm{~d}^{6}\left({ }^{3} \mathrm{H}\right) 5 \mathrm{~d}$ & ${ }^{4} \mathrm{~K}$ & $15 / 2$ & 124297.017 & -5.220 \\
$3 \mathrm{~d}^{6}\left({ }^{3} \mathrm{H}\right) 5 \mathrm{~d}$ & ${ }^{4} \mathrm{I}$ & $15 / 2$ & 124357.304 & +12.292 \\
$3 \mathrm{~d}^{6}\left({ }^{3} \mathrm{H}\right) 5 \mathrm{~d}$ & ${ }^{4} \mathrm{~K}$ & $13 / 2$ & 124415.353 & -14.256 \\
$3 \mathrm{~d}^{6}\left({ }^{3} \mathrm{H}\right) 5 \mathrm{~d}$ & ${ }^{2} \mathrm{I}$ & $11 / 2$ & 124976.008 & -38.096 \\
$3 \mathrm{~d}^{6}\left({ }^{3} \mathrm{~F}\right) 5 \mathrm{~d}$ & ${ }^{4} \mathrm{H}$ & $13 / 2$ & 125732.991 & +9.243 \\
& & & & \\
$3 \mathrm{~d}^{6}\left({ }^{5} \mathrm{D}\right) 6 \mathrm{~d}$ & ${ }^{6} \mathrm{D}$ & $5 / 2$ & 113934.466 & -58.836 \\
$3 \mathrm{~d}^{6}\left({ }^{5} \mathrm{D}\right) 6 \mathrm{~d}$ & ${ }^{4} \mathrm{D}$ & $7 / 2$ & 114009.934 & -3.477 \\
$3 \mathrm{~d}^{6}\left({ }^{5} \mathrm{D}\right) 6 \mathrm{~d}$ & ${ }^{6} \mathrm{G}$ & $7 / 2$ & 114428.399 & +51.787 \\
$3 \mathrm{~d}^{6}\left({ }^{5} \mathrm{D}\right) 6 \mathrm{~d}$ & ${ }^{6} \mathrm{G}$ & $5 / 2$ & 114619.007 & +22.415 \\
\hline
\end{tabular}

either to a too low log $g f$ value or to some unknown component which increases the line intensity. When lines are computed much stronger than the observed ones, some problem with the energy levels or/and $\log g f$ computations is very probably present. When we observed a very good agreement between the observed and computed lines, either isolated or blends, we added the note "good agreement".

Figure 1 shows the Fe II spectrum in the 5185-5196 $\AA$ interval, computed before and after the determination of the new energy levels. Figure 2 compares the observed spectrum of
HR 6000 with the synthetic spectrum computed with the line list including the new Fe II lines. When the two figures are considered together, the improvement in the comparison between the observed and computed spectra is evident.

\section{Conclusions}

Computed atomic data and stellar spectra observed at high resolution and high signal-to-noise ratio of the iron-overabundant, slow-rotating star HR 6000 were used to extend laboratory studies on Fe II energy levels and line transitions. We identified as Fe II about 500 unidentified spectral lines in the 3800-8000 region. A few of these lines were already identified as iron from laboratory analyses (Johansson 2007, private communication), but they were never classified. Because numerous other new lines are components of blends they contribute to improve the agreement between observed and computed spectra. On the other hand, there is a small number of new lines which are not observed in the spectrum. We believe that they are due to computational problems related with the mixing of the even energy levels rather than to incorrect energy values for the new 4 f odd levels.

In spite of the large number of the new identified lines, several medium-strong lines and a conspicuous number of weak lines remain still unidentified in the spectral region we analyzed. If we examine the list of the Fe II lines which correspond to transitions from predicted energy levels, we can count about 4600 lines with $\log g f \geq-1.0$, where about 400 of them have $\log g f \geq 0.0$. Because the transitions producing these lines occur between high-excitation energy levels that are not strongly populated, most of the lines are weak in a star like HR 6000. This 

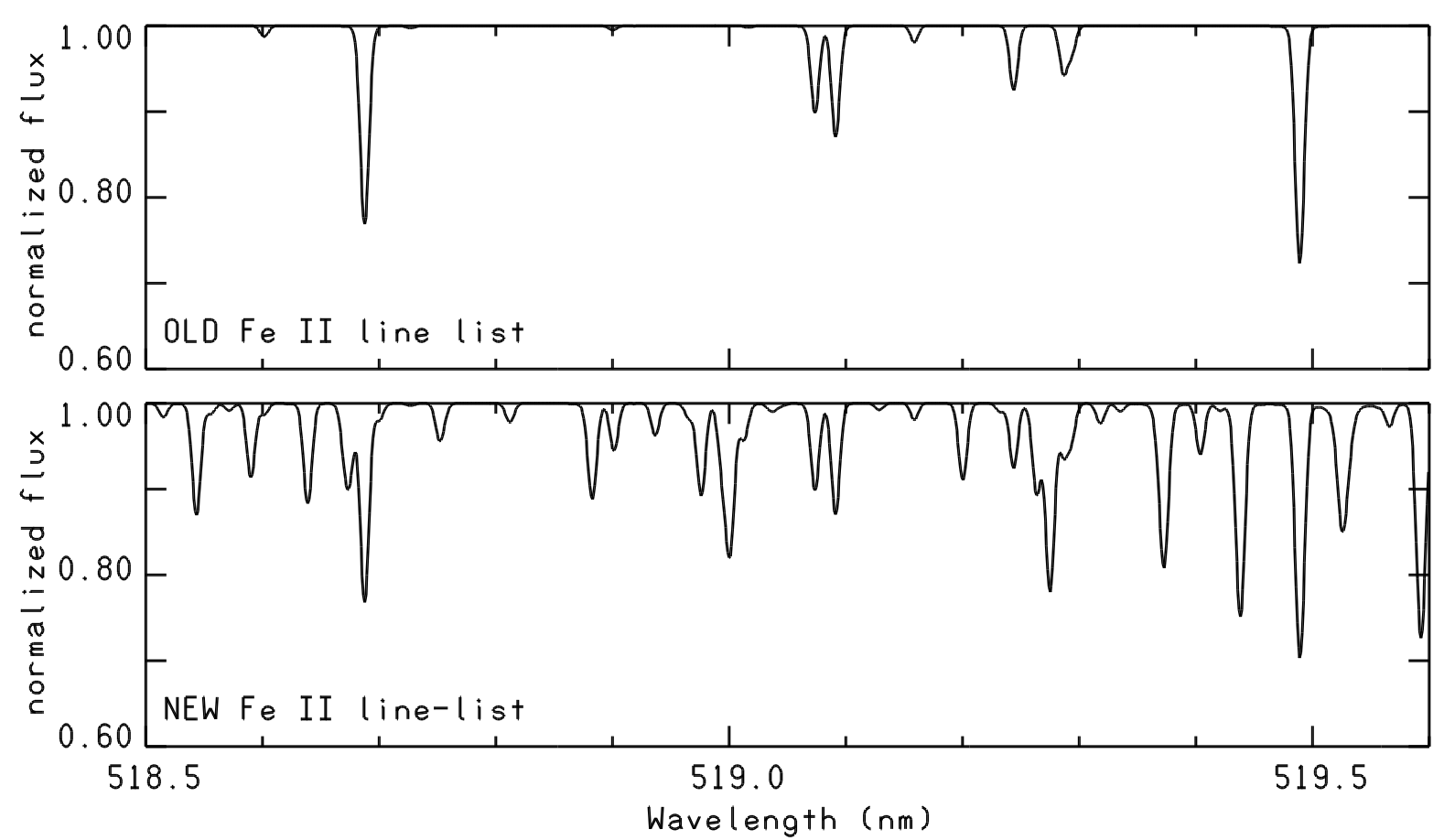

Fig. 1. Upper panel shows the Fe II synthetic spectrum for the parameters of HR $6000\left(T_{\text {eff }}=13450 \mathrm{~K}, \log g=4.3, v \sin i=1.5 \mathrm{~km}{ }^{-1}\right.$, $[\mathrm{Fe} / \mathrm{H}]=$ +0.9 ) computed with the line list availble before this work. The lower panel is the same, but with the new Fe II lines added in the line list.

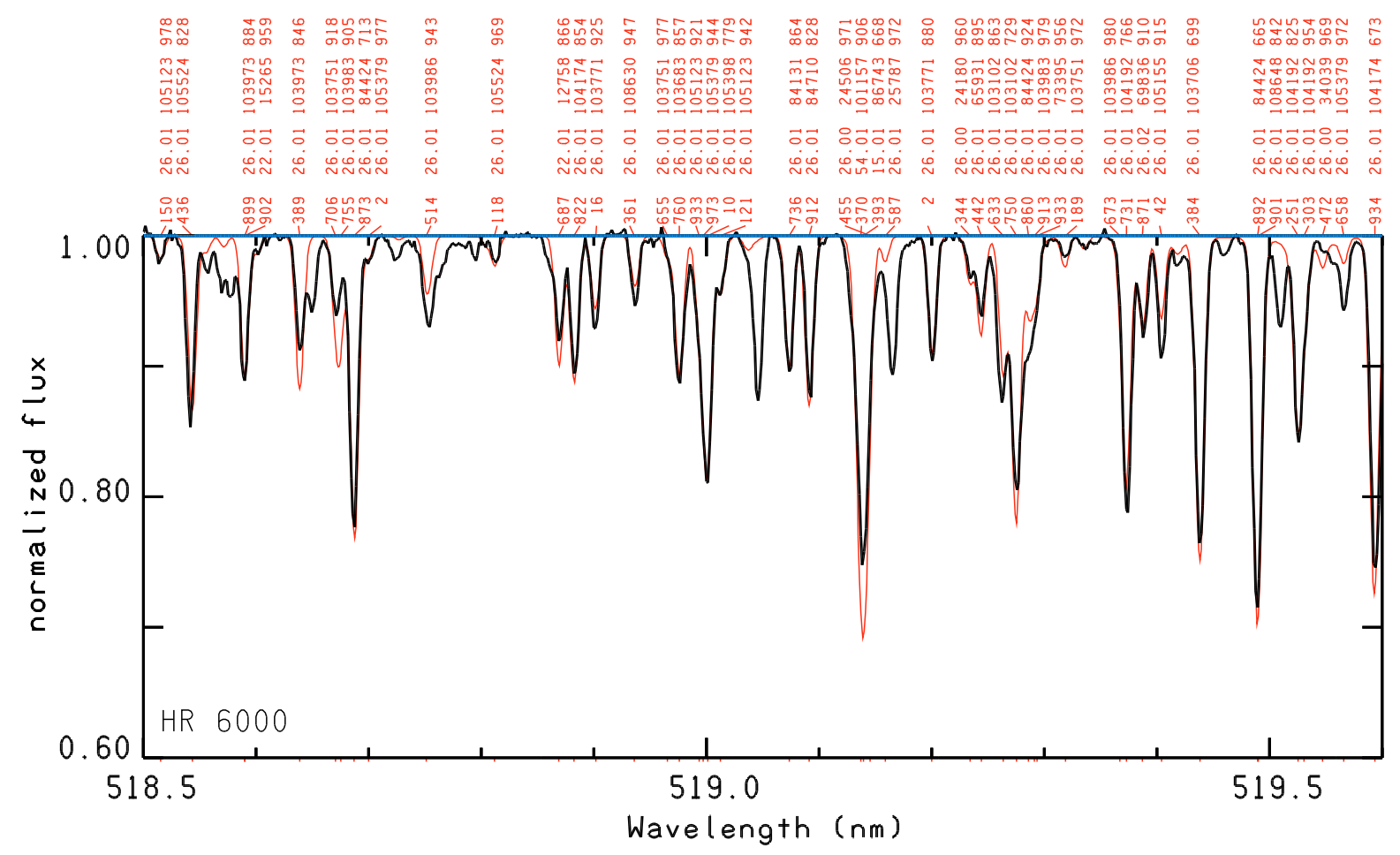

Fig. 2. Comparison of the UVES spectrum of HR 6000 (black line) with a synthetic spectrum (red line) computed with a line list including the new Fe II lines. The line identification can be decoded as follows: for the first line, 150 last 3 digits of wavelength $518.5150 \mathrm{~nm}$; 26 atomic number of iron; .01 charge/100, i.e. 26.01 identifies the line as Fe II; 105123 is the energy of the lower level in $\mathrm{cm}^{-1}$; 970 is the residual central intensity in per mil. 
large number of weak predicted lines could explain the spectrum of HR 6000 longward of about $5800 \AA$. The spectrum looks like it is affected by a noise larger than that due to the instrumental effects. Castelli \& Hubrig (2007) explained this "noise" with the presence of a T-Tauri star affecting the HR 6000 spectrum. After this study, we prefer to state that the spectrum shows the presence of numerous weak Fe II lines from high-excitation levels, probably $4 \mathrm{~d}, 5 \mathrm{~d}, 6 \mathrm{~d}-4 \mathrm{f}, 5 \mathrm{f}, 6 \mathrm{f}$ transitions, which still have to be identified. The hypothesis of the presence of the T-Tauri star affecting the HR 6000 spectrum is an example of an incorrect conclusion that can be drawn owing to the use of incomplete line lists. We will extend this study of the Fe II spectrum to the near infrared region in the near future using CRIRES (CRyogenic high-resolution InfraRed Echelle Spectrograph) observations of HR 6000 and 46 Aql. The observations are scheduled in summer 2010 (ESO proposal 41380, P.I. S. Hubrig).

\section{References}

Adam, J., Baschek, B., Johansson, S., Nilsson, A. E., \& Brage, T. 1987, ApJ, 312,337

Biémont, E., Johansson, S., \& Palmeri, P. 1997, Phys. Scr., 55, 559

Castelli, F., Kurucz, R., \& Hubrig, S. 2009, A\&A, 508, 401, Paper I

Castelli, F., \& Hubrig, S. 2007, A\&A, 475, 1041

Castelli, F., Johansson, S., \& Hubrig, S. 2008, J. Phys. Conf. Ser., 130, 012003

Cowan, R. D. 1981, The Theory of Atomic Structure and Spectra (Berkeley: Univ. California Press)

Johansson, S. 1978, Phys. Scr., 18, 217

Johansson, S., \& Baschek, B. 1988, Nucl. Instr. Meth. Phys. Res. B, 31, 222 Johansson, S. 2009, Phys. Scr. T, 134, 014013

Kurucz, R. L. 2005, Mem. Soc. Astron. Ital. Supp., 8, 14

Kurucz, R. L. 2009, Am. Inst. Phys. Conf. Ser., 1171, 43

Rosberg, M., \& Johansson, S. 1992, Phys. Scr., 45, 590

Sugar, J., \& Corliss, C. 1985, J. Phys. Chem. Ref. Data, 14, Supp., 2 
F. Castelli and R. L. Kurucz: New Fe II energy levels from stellar spectra

Table 6. Fe II lines in the 3800-8000 A region with $\log g f \geq-1.5$ and $3 \mathrm{~d}^{6}\left({ }^{3} \mathrm{P}\right) 4 \mathrm{f}$ energy levels as upper levels.

\begin{tabular}{|c|c|c|c|c|c|c|c|c|c|}
\hline \multicolumn{4}{|c|}{ Upper level } & \multicolumn{2}{|c|}{ Lower level } & \multirow{2}{*}{$\begin{array}{c}\lambda(\text { calc }) \\
\AA\end{array}$} & \multirow{2}{*}{$\begin{array}{c}\log g f \\
\text { KUR }\end{array}$} & \multirow{2}{*}{$\begin{array}{c}\lambda(\mathrm{obs}) \\
\AA \\
\end{array}$} & \multirow[t]{2}{*}{ Notes } \\
\hline $\mathrm{cm}^{-1}$ & & & $J$ & $\mathrm{~cm}^{-1}$ & & & & & \\
\hline 122351.810 & $\left({ }^{3} \mathrm{P}\right) 4 \mathrm{f}$ & $2[5]$ & $11 / 2$ & $\begin{array}{l}103165.320 \\
103683.070 \\
103771.320 \\
104807.210 \\
104916.550 \\
106722.170 \\
109811.920\end{array}$ & $\begin{array}{l}\left({ }^{3} \mathrm{P}\right) 4 d^{4} \mathrm{~F}_{9 / 2} \\
\left({ }^{5} \mathrm{D}\right) 5 \mathrm{~d}^{4} \mathrm{~F}_{9 / 2} \\
\left({ }^{3} \mathrm{H}\right) 4 \mathrm{~d}^{4} \mathrm{G}_{9 / 2} \\
\left({ }^{3} \mathrm{H}\right) 4 \mathrm{~d}^{2} \mathrm{G}_{9 / 2} \\
\left({ }^{3} \mathrm{H}\right) 4 \mathrm{~d}^{4} \mathrm{~F}_{9 / 2} \\
\left({ }^{3} \mathrm{~F}\right) 4 \mathrm{~d}^{4} \mathrm{~F}_{9 / 2} \\
\left({ }^{3} \mathrm{G}\right) 4 \mathrm{~d}^{4} \mathrm{~F}_{9 / 2}\end{array}$ & $\begin{array}{l}5210.550 \\
5355.059 \\
5380.493 \\
5698.178 \\
5733.913 \\
6396.332 \\
7972.359\end{array}$ & $\begin{array}{l}+0.795 \\
+0.164 \\
-1.047 \\
-0.539 \\
-0.635 \\
-0.741 \\
-0.985\end{array}$ & $\begin{array}{l}5210.55 \\
5355.06 \\
\\
5733.90 \\
6396.32\end{array}$ & $\begin{array}{l}\text { good agreement } \\
\text { computed too strong } \\
\text { at the noise level } \\
\text { blend with a telluric line } \\
\text { computed too weak } \\
\text { computed too weak } \\
\text { at the noise level }\end{array}$ \\
\hline 122324.142 & $\left({ }^{3} \mathrm{P}\right) 4 \mathrm{f}$ & $2[5]$ & $9 / 2$ & $\begin{array}{l}103102.860 \\
103191.917 \\
103986.330 \\
104107.950 \\
104481.590 \\
105123.000 \\
105775.491\end{array}$ & $\begin{array}{l}\left({ }^{3} \mathrm{P}\right) 4 d^{4} \mathrm{D}_{7 / 2} \\
\left({ }^{3} \mathrm{P}\right) 4 \mathrm{~d}^{2} \mathrm{~F}_{7 / 2} \\
\left({ }^{3} \mathrm{H}\right) 4 d^{4} \mathrm{H}_{7 / 2} \\
\left({ }^{3} \mathrm{P}\right) 4 d^{4} \mathrm{~F}_{7 / 2} \\
\left({ }^{3} \mathrm{H}\right) 4 d^{2} \mathrm{~F}_{7 / 2} \\
\left({ }^{3} \mathrm{H}\right) 4 d^{2} \mathrm{G}_{7 / 2} \\
\left({ }^{3} \mathrm{~F}\right) 4 d^{2} \mathrm{~F}_{7 / 2}\end{array}$ & $\begin{array}{l}5201.118 \\
5225.329 \\
5451.698 \\
5488.097 \\
5603.024 \\
5811.956 \\
6041.116\end{array}$ & $\begin{array}{l}-0.056 \\
+0.634 \\
-1.133 \\
-0.362 \\
-0.170 \\
-1.441 \\
-0.837\end{array}$ & $\begin{array}{l}5603.05 \\
6041.1\end{array}$ & $\begin{array}{l}\text { wrong,not observed } \\
\text { blend, good agreement } \\
\text { blend, good agreement } \\
\text { blend, good agreement } \\
\text { blend,good agreement } \\
\text { weak,good agreement }\end{array}$ \\
\hline 122355.116 & $\left({ }^{3} \mathrm{P}\right) 4 \mathrm{f}$ & $2[4]$ & $9 / 2$ & $\begin{array}{l}102394.718 \\
103102.860 \\
103165.320 \\
103191.917 \\
103683.070 \\
104107.950 \\
104807.210 \\
106767.210\end{array}$ & $\begin{array}{l}\left({ }^{5} \mathrm{D}\right) 6 \mathrm{~s}{ }^{4} \mathrm{D}_{7 / 2} \\
\left({ }^{3} \mathrm{P}\right) 4 \mathrm{~d}^{4} \mathrm{D}_{7 / 2} \\
\left({ }^{3} \mathrm{P}\right) 4 \mathrm{~d}^{4} \mathrm{~F}_{9 / 2} \\
\left({ }^{3} \mathrm{P}\right) 4 \mathrm{~d}^{2} \mathrm{~F}_{7 / 2} \\
\left({ }^{5} \mathrm{D}\right) 5 \mathrm{~d}^{4} \mathrm{~F}_{9 / 2} \\
\left({ }^{3} \mathrm{P}\right) 4 \mathrm{~d}^{4} \mathrm{~F}_{7 / 2} \\
\left({ }^{3} \mathrm{H}\right) 4 \mathrm{~d}^{2} \mathrm{G}_{9 / 2} \\
\left({ }^{3} \mathrm{~F}\right) 4 \mathrm{~d}^{4} \mathrm{~F}_{7 / 2}\end{array}$ & $\begin{array}{l}5008.523 \\
5192.750 \\
5209.652 \\
5216.883 \\
5354.110 \\
5478.781 \\
5697.105 \\
6413.457\end{array}$ & $\begin{array}{l}-0.809 \\
+0.657 \\
-0.035 \\
-0.404 \\
-0.637 \\
-1.319 \\
-1.443 \\
-1.407\end{array}$ & $\begin{array}{l}5192.75 \\
5209.66 \\
5354.1\end{array}$ & $\begin{array}{l}\text { weak, computed too strong } \\
\text { lab, good agreement } \\
\text { lab, good agreement } \\
\text { blend } \\
\text { weak } \\
\text { at the continuum level } \\
\text { at the continuum level } \\
\text { blend }\end{array}$ \\
\hline 122355.550 & $\left({ }^{3} \mathrm{P}\right) 4 \mathrm{f}$ & $2[4]$ & $7 / 2$ & $\begin{array}{l}102394.718 \\
102802.312 \\
103002.670 \\
103102.860 \\
103165.320 \\
103191.917 \\
106796.660\end{array}$ & $\begin{array}{l}\left({ }^{5} \mathrm{D}\right) 6 \mathrm{~s}{ }^{4} \mathrm{D}_{7 / 2} \\
\left({ }^{5} \mathrm{D}\right) 6 \mathrm{~s}{ }^{4} \mathrm{D}_{5 / 2} \\
\left({ }^{3} \mathrm{P}\right) 4 \mathrm{~d}^{4} \mathrm{D}_{5 / 2} \\
\left({ }^{3} \mathrm{P}\right) 4 \mathrm{~d}^{4} \mathrm{D}_{7 / 2} \\
\left({ }^{3} \mathrm{P}\right) 4 \mathrm{~d}^{4} \mathrm{~F}_{9 / 2} \\
\left({ }^{3} \mathrm{P}\right) 4 \mathrm{~d}^{2} \mathrm{~F}_{7 / 2} \\
\left({ }^{3} \mathrm{~F}\right) 4 \mathrm{~d}{ }^{4} \mathrm{P}_{5 / 2}\end{array}$ & $\begin{array}{l}5008.414 \\
5112.818 \\
5165.751 \\
5192.633 \\
5209.534 \\
5216.765 \\
6425.418\end{array}$ & $\begin{array}{l}-1.258 \\
-0.959 \\
+0.441 \\
+0.155 \\
-1.105 \\
-0.764 \\
-1.436\end{array}$ & $\begin{array}{l}5112.82 \\
5165.75 \\
5192.62\end{array}$ & $\begin{array}{l}\text { good agreement } \\
\text { computed too weak } \\
\text { lab, good agreement } \\
\text { lab, computed too weak } \\
\text { blend, good agreement } \\
\text { blend } \\
\text { at the continuum level }\end{array}$ \\
\hline 122351.488 & $\left({ }^{3} \mathrm{P}\right) 4 \mathrm{f}$ & $2[3]$ & $7 / 2$ & $\begin{array}{l}103102.860 \\
103191.917 \\
103597.402 \\
104023.910 \\
104107.950 \\
104481.590 \\
104569.230 \\
105234.237 \\
107407.800\end{array}$ & $\begin{array}{l}\left({ }^{3} \mathrm{P}\right) 4 \mathrm{~d}^{4} \mathrm{D}_{7 / 2} \\
\left({ }^{3} \mathrm{P}\right) 4 \mathrm{~d}^{2} \mathrm{~F}_{7 / 2} \\
\left({ }^{3} \mathrm{P}\right) 4 \mathrm{~d}^{2} \mathrm{D}_{5 / 2} \\
\left({ }^{3} \mathrm{H}\right) 4 \mathrm{~d}^{4} \mathrm{G}_{5 / 2} \\
\left({ }^{3} \mathrm{P}\right) 4 \mathrm{~d}^{4} \mathrm{~F}_{7 / 2} \\
\left({ }^{3} \mathrm{H}\right) 4 \mathrm{~d}^{2} \mathrm{~F}_{7 / 2} \\
\left({ }^{3} \mathrm{P}\right) 4 \mathrm{~d}^{4} \mathrm{~F}_{5 / 2} \\
\left({ }^{3} \mathrm{H}\right) 4 \mathrm{~d}^{4} \mathrm{~F}_{5 / 2} \\
\left({ }^{3} \mathrm{~F}\right) 4 \mathrm{~d}^{2} \mathrm{D}_{5 / 2}\end{array}$ & $\begin{array}{l}5193.729 \\
5217.871 \\
5330.689 \\
5454.742 \\
5479.870 \\
5594.450 \\
5622.022 \\
5840.440 \\
6689.941\end{array}$ & $\begin{array}{l}-1.320 \\
-0.250 \\
+0.525 \\
-1.327 \\
-1.320 \\
-1.116 \\
-0.573 \\
-1.282 \\
-0.330\end{array}$ & $\begin{array}{l}5217.870 \\
5330.680 \\
\\
5594.42 \\
5622.02 \\
6689.91\end{array}$ & $\begin{array}{l}\text { blend } \\
\text { lab } \\
\text { lab } \\
\text { at the continuum level } \\
\text { at the continuum level } \\
\text { computed too weak? } \\
\text { computed too weak? } \\
\text { at the continuum level }\end{array}$ \\
\hline 123629.520 & $\left({ }^{3} \mathrm{P}\right) 4 \mathrm{f}$ & $1[4]$ & $9 / 2$ & $\begin{array}{l}103102.860 \\
104000.810 \\
104107.950 \\
104481.590 \\
104873.230 \\
104993.860 \\
105123.000 \\
105220.600 \\
105775.491 \\
106767.210 \\
110167.280\end{array}$ & $\begin{array}{l}\left({ }^{3} \mathrm{P}\right) 4 \mathrm{~d}^{4} \mathrm{D}_{7 / 2} \\
\left({ }^{5} \mathrm{D}\right) 5 \mathrm{~d}^{6} \mathrm{P}_{7 / 2} \\
\left({ }^{3} \mathrm{P}\right) 4 \mathrm{~d}^{4} \mathrm{~F}_{7 / 2} \\
\left({ }^{3} \mathrm{H}\right) 4 \mathrm{~d}^{2} \mathrm{~F}_{7 / 2} \\
\left({ }^{5} \mathrm{D}\right) 5 \mathrm{~d}^{4} \mathrm{D}_{7 / 2} \\
\left({ }^{3} \mathrm{~F}\right) 4 \mathrm{~d}^{4} \mathrm{D}_{7 / 2} \\
\left({ }^{3} \mathrm{H}\right) 4 \mathrm{~d}^{2} \mathrm{G}_{7 / 2} \\
\left({ }^{3} \mathrm{H}\right) 4 \mathrm{~d}^{4} \mathrm{~F}_{7 / 2} \\
\left({ }^{3} \mathrm{~F}\right) 4 \mathrm{~d}^{2} \mathrm{~F}_{7 / 2} \\
\left({ }^{3} \mathrm{~F}\right) 4 \mathrm{~d}^{4} \mathrm{~F}_{7 / 2} \\
\left({ }^{3} \mathrm{G}\right) 4 \mathrm{~d}^{4} \mathrm{~F}_{7 / 2}\end{array}$ & $\begin{array}{l}4870.353 \\
5093.159 \\
5121.112 \\
5221.043 \\
5330.062 \\
5364.564 \\
5401.999 \\
5430.640 \\
5599.422 \\
5928.743 \\
7426.139\end{array}$ & $\begin{array}{l}-1.402 \\
-0.981 \\
+0.327 \\
+0.408 \\
-1.183 \\
-0.118 \\
-0.418 \\
-1.066 \\
-0.624 \\
-0.677 \\
-1.173\end{array}$ & $\begin{array}{l}5121.1 \\
5221.04 \\
5364.55 \\
\\
5430.64 \\
5599.42 \\
5928.72\end{array}$ & $\begin{array}{l}\text { at the continuum level } \\
\text { blend } \\
\text { lab, good agreement } \\
\text { lab, good agreement } \\
\text { blend } \\
\text { computed too strong } \\
\text { blend } \\
\text { computed too weak } \\
\text { good agreement } \\
\text { at the noise level }\end{array}$ \\
\hline
\end{tabular}


Table 6. continued.

\begin{tabular}{|c|c|c|c|c|c|c|c|c|c|}
\hline \multicolumn{4}{|c|}{ Upper level } & \multicolumn{2}{|c|}{ Lower level } & \multirow{2}{*}{$\begin{array}{c}\lambda(\text { calc }) \\
\AA\end{array}$} & \multirow{2}{*}{$\begin{array}{c}\log g f \\
\text { KUR }\end{array}$} & \multirow{2}{*}{$\begin{array}{c}\lambda(\mathrm{obs}) \\
\AA\end{array}$} & \multirow[t]{2}{*}{ Notes } \\
\hline $\mathrm{cm}^{-1}$ & & & $J$ & $\mathrm{~cm}^{-1}$ & & & & & \\
\hline 123637.833 & $\left({ }^{3} \mathrm{P}\right) 4 \mathrm{f}$ & $1[4]$ & $7 / 2$ & $\begin{array}{l}102802.312 \\
103002.670 \\
103597.402 \\
104107.950 \\
104120.270 \\
104481.590 \\
104569.230 \\
104993.860 \\
105127.770 \\
105234.237 \\
105379.430 \\
105711.730 \\
106208.560 \\
106796.660 \\
106866.760 \\
107407.800 \\
110428.280\end{array}$ & $\begin{array}{l}\left({ }^{5} \mathrm{D}\right) 6 \mathrm{~s}^{4} \mathrm{D}_{5 / 2} \\
\left({ }^{3} \mathrm{P}\right) 4 \mathrm{~d}^{4} \mathrm{D}_{5 / 2} \\
\left({ }^{3} \mathrm{P}\right) 4 \mathrm{~d}^{2} \mathrm{D}_{5 / 2} \\
\left({ }^{3} \mathrm{P}\right) 4 \mathrm{~d}^{4} \mathrm{~F}_{7 / 2} \\
\left({ }^{5} \mathrm{D}\right) 5 \mathrm{~d}^{6} \mathrm{P}_{5 / 2} \\
\left({ }^{3} \mathrm{H}\right) 4 \mathrm{~d}^{2} \mathrm{~F}_{7 / 2} \\
\left({ }^{3} \mathrm{P}\right) 4 \mathrm{~d}^{4} \mathrm{~F}_{5 / 2} \\
\left({ }^{3} \mathrm{~F}\right) 4 \mathrm{~d}^{4} \mathrm{D}_{7 / 2} \\
\left({ }^{5} \mathrm{D}\right) 5 \mathrm{~d}^{4} \mathrm{D}_{5 / 2} \\
\left({ }^{3} \mathrm{H}\right) 4 \mathrm{~d}^{4} \mathrm{~F}_{5 / 2} \\
\left({ }^{3} \mathrm{~F}\right) 4 \mathrm{~d}^{4} \mathrm{D}_{5 / 2} \\
\left({ }^{5} \mathrm{D}\right) 5 \mathrm{~d}^{6} \mathrm{~S}_{5 / 2} \\
\left({ }^{3} \mathrm{~F}\right) 4 \mathrm{~d}^{2} \mathrm{~F}_{5 / 2} \\
\left({ }^{3} \mathrm{~F}\right) 4 \mathrm{~d}^{4} \mathrm{P}_{5 / 2} \\
\left({ }^{3} \mathrm{~F}\right) 4 \mathrm{~d}^{4} \mathrm{~F}_{5 / 2} \\
\left({ }^{3} \mathrm{~F}\right) 4 \mathrm{~d}^{2} \mathrm{D}_{5 / 2} \\
\left({ }^{3} \mathrm{G}\right) 4 \mathrm{~d}^{4} \mathrm{~F}_{5 / 2}\end{array}$ & $\begin{array}{l}4798.155 \\
4844.743 \\
4988.521 \\
5118.932 \\
5122.163 \\
5218.777 \\
5242.763 \\
5362.172 \\
5400.965 \\
5432.211 \\
5475.409 \\
5576.909 \\
5735.883 \\
5936.184 \\
5960.996 \\
6159.712 \\
7568.195\end{array}$ & $\begin{array}{l}-1.297 \\
-0.954 \\
-0.339 \\
-0.819 \\
-1.282 \\
-0.644 \\
+0.180 \\
-1.268 \\
-1.143 \\
-0.531 \\
-0.552 \\
-1.432 \\
-1.221 \\
-1.317 \\
-0.565 \\
-0.665 \\
-1.229\end{array}$ & $\begin{array}{l}4988.51 \\
5118.95 \\
5242.775\end{array}$ & $\begin{array}{l}\text { at the continuum level } \\
\text { computed too strong } \\
\text { lab } \\
\text { lab, computed too weak } \\
\text { blend } \\
\text { lab } \\
\text { at the continuum level } \\
\text { at the continuum level } \\
\text { wrong, not observed } \\
\text { computed too strong } \\
\text { at the continuum level } \\
\text { at the continuum level } \\
\text { at the level of the noise } \\
\text { blend? } \\
\text { no spectrum }\end{array}$ \\
\hline 123615.875 & $\left({ }^{3} \mathrm{P}\right) 4 \mathrm{f}$ & $1[3]$ & $7 / 2$ & $\begin{array}{l}103597.402 \\
104023.910 \\
104107.950 \\
104120.270 \\
104209.610 \\
104481.590 \\
104569.230 \\
105127.770 \\
105234.237 \\
106208.560\end{array}$ & $\begin{array}{l}\left({ }^{3} \mathrm{P}\right) 4 \mathrm{~d}^{2} \mathrm{D}_{5 / 2} \\
\left({ }^{3} \mathrm{H}\right) 4 \mathrm{~d}^{4} \mathrm{G}_{5 / 2} \\
\left({ }^{3} \mathrm{P}\right) 4 \mathrm{~d}^{4} \mathrm{~F}_{7 / 2} \\
\left({ }^{5} \mathrm{D}\right) 5 \mathrm{~d}^{6} \mathrm{P}_{5 / 2} \\
\left({ }^{3} \mathrm{H}\right) 4 \mathrm{~d}^{2} \mathrm{~F}_{5 / 2} \\
\left({ }^{3} \mathrm{H}\right) 4 \mathrm{~d}^{2} \mathrm{~F}_{7 / 2} \\
\left({ }^{3} \mathrm{P}\right) 4 \mathrm{~d}^{4} \mathrm{~F}_{5 / 2} \\
\left({ }^{5} \mathrm{D}\right) 5 \mathrm{~d}^{4} \mathrm{D}_{5 / 2} \\
\left({ }^{3} \mathrm{H}\right) 4 \mathrm{~d}^{4} \mathrm{~F}_{5 / 2} \\
\left({ }^{3} \mathrm{~F}\right) 4 \mathrm{~d}^{2} \mathrm{~F}_{5 / 2}\end{array}$ & $\begin{array}{l}4993.993 \\
5102.711 \\
5124.694 \\
5127.932 \\
5151.540 \\
5224.766 \\
5248.807 \\
5407.380 \\
5438.700 \\
5743.118\end{array}$ & $\begin{array}{l}-1.435 \\
-0.526 \\
-1.046 \\
-0.244 \\
-0.081 \\
-0.973 \\
-0.232 \\
-1.391 \\
-0.416 \\
-0.454\end{array}$ & $\begin{array}{l}5102.7 \\
5124.69 \\
\\
5151.52 \\
5227.77 \\
5248.801 \\
5407.37 \\
5438.70 \\
5743.10\end{array}$ & $\begin{array}{l}\text { lab, good agreement } \\
\text { good agreement } \\
\text { wrong, not obs } \\
\text { J78, lab, computed too weak } \\
\text { good agreement } \\
\text { computed too strong } \\
\text { computed too weak } \\
\text { computed too strong } \\
\text { good agreement }\end{array}$ \\
\hline 123649.493 & $\left({ }^{3} \mathrm{P}\right) 4 \mathrm{f}$ & $1[3]$ & $5 / 2$ & $\begin{array}{l}104209.610 \\
104569.230 \\
104572.920 \\
104588.710 \\
104839.998 \\
105234.237 \\
105317.440 \\
105518.140 \\
106846.650 \\
107430.250 \\
108105.900\end{array}$ & $\begin{array}{l}\left({ }^{3} \mathrm{H}\right) 4 \mathrm{~d}^{2} \mathrm{~F}_{5 / 2} \\
\left({ }^{3} \mathrm{P}\right) 4 \mathrm{~d}^{4} \mathrm{~F}_{5 / 2} \\
\left({ }^{3} \mathrm{P}\right) 4 \mathrm{~d}^{4} \mathrm{~F}_{3 / 2} \\
\left({ }^{5} \mathrm{D}\right) 5 \mathrm{~d}^{6} \mathrm{D}_{3 / 2} \\
\left({ }^{3} \mathrm{P}\right) 4 \mathrm{~d}^{2} \mathrm{D}_{3 / 2} \\
\left({ }^{3} \mathrm{H}\right) 4 \mathrm{~d}^{4} \mathrm{~F}_{5 / 2} \\
\left({ }^{3} \mathrm{P}\right) 4 \mathrm{~d}^{2} \mathrm{P}_{3 / 2} \\
\left({ }^{3} \mathrm{H}\right) 4 \mathrm{~d}^{4} \mathrm{~F}_{3 / 2} \\
\left({ }^{3} \mathrm{~F}\right) 4 \mathrm{~d}^{4} \mathrm{~F}_{3 / 2} \\
\left({ }^{3} \mathrm{~F}\right) 4 \mathrm{~d}^{2} \mathrm{D}_{3 / 2} \\
\left({ }^{3} \mathrm{~F}\right) 4 \mathrm{~d}^{2} \mathrm{P}_{3 / 2}\end{array}$ & $\begin{array}{l}5142.631 \\
5239.559 \\
5240.573 \\
5244.914 \\
5314.985 \\
5428.771 \\
5453.411 \\
5513.777 \\
5949.725 \\
6163.810 \\
6431.741\end{array}$ & $\begin{array}{l}-1.288 \\
-1.150 \\
+0.071 \\
-1.288 \\
-0.441 \\
-1.471 \\
+0.082 \\
-0.591 \\
-1.358 \\
-0.253 \\
-0.724\end{array}$ & $\begin{array}{l}5239.56 \\
5240.587\end{array}$ & $\begin{array}{l}\text { at the continuum level } \\
\text { good agreement } \\
\text { lab, good agreement } \\
\text { blend } \\
\text { blend,computed too strong } \\
\text { blend } \\
\text { lab, computed too strong } \\
\text { wrong, not observed } \\
\text { at the continuum level } \\
\text { wrong, not observed } \\
\text { blend }\end{array}$ \\
\hline 124157.060 & $\left({ }^{3} \mathrm{P}\right) 4 \mathrm{f}$ & $0[3]$ & $5 / 2$ & $\begin{array}{l}104569.230 \\
104572.920 \\
104588.710 \\
104839.998 \\
105234.237 \\
105317.440 \\
105460.230 \\
105518.140 \\
106846.650 \\
107430.250 \\
108105.900 \\
110609.540\end{array}$ & $\begin{array}{l}\left({ }^{3} \mathrm{P}\right) 4 \mathrm{~d}^{4} \mathrm{~F}_{5 / 2} \\
\left({ }^{3} \mathrm{P}\right) 4 \mathrm{~d}^{4} \mathrm{~F}_{3 / 2} \\
\left({ }^{5} \mathrm{D}\right) 5 \mathrm{~d}^{6} \mathrm{D}_{3 / 2} \\
\left({ }^{3} \mathrm{P}\right) 4 \mathrm{~d}^{2} \mathrm{D}_{3 / 2} \\
\left({ }^{3} \mathrm{H}\right) 4 \mathrm{~d}^{4} \mathrm{~F}_{5 / 2} \\
\left({ }^{3} \mathrm{P}\right) 4 \mathrm{~d}^{2} \mathrm{P}_{3 / 2} \\
\left({ }^{3} \mathrm{~F}\right) 4 \mathrm{~d}^{4} \mathrm{D}_{3 / 2} \\
\left({ }^{3} \mathrm{H}\right) 4 \mathrm{~d}^{4} \mathrm{~F}_{3 / 2} \\
\left({ }^{3} \mathrm{~F}\right) 4 \mathrm{~d}^{4} \mathrm{~F}_{3 / 2} \\
\left({ }^{3} \mathrm{~F}\right) 4 \mathrm{~d}^{2} \mathrm{D}_{3 / 2} \\
\left({ }^{3} \mathrm{~F}\right) 4 \mathrm{~d}^{2} \mathrm{P}_{3 / 2} \\
\left({ }^{3} \mathrm{G}\right) 4 \mathrm{~d}^{4} \mathrm{~F}_{3 / 2}\end{array}$ & $\begin{array}{l}5103.788 \\
5104.750 \\
5108.869 \\
5175.329 \\
5283.154 \\
5306.486 \\
5347.013 \\
5363.626 \\
5775.269 \\
5976.771 \\
6228.356 \\
7379.392 \\
\end{array}$ & $\begin{array}{l}-1.191 \\
+0.094 \\
-1.369 \\
-1.125 \\
-0.937 \\
-1.020 \\
-0.482 \\
+0.082 \\
-0.286 \\
-0.922 \\
-0.686 \\
-1.370\end{array}$ & $\begin{array}{l}5306.49 \\
5347.05 \\
5363.61 \\
5775.25 \\
\\
6228.34\end{array}$ & $\begin{array}{l}\text { good agreement } \\
\text { lab, good agreement } \\
\text { blend } \\
\text { blend } \\
\text { computed too weak } \\
\text { blend } \\
\text { computed too strong } \\
\text { good agreement } \\
\text { blend } \\
\text { good agreement } \\
\text { at the continuum level }\end{array}$ \\
\hline
\end{tabular}


F. Castelli and R. L. Kurucz: New Fe II energy levels from stellar spectra

Table 7. Fe II lines in the $3800-8000 \AA$ A region with $\log g f \geq-1.5$ and $3 \mathrm{~d}^{6}\left({ }^{3} \mathrm{H}\right) 4 \mathrm{f}$ energy level as upper levels.

\begin{tabular}{|c|c|c|c|c|c|c|c|c|c|}
\hline \multicolumn{4}{|c|}{ Upper level } & \multicolumn{2}{|c|}{ Lower level } & \multirow{2}{*}{$\begin{array}{c}\lambda(\text { calc }) \\
\AA \\
\end{array}$} & \multirow{2}{*}{$\begin{array}{c}\log g f \\
\text { KUR }\end{array}$} & \multirow{2}{*}{$\begin{array}{c}\lambda(\text { obs }) \\
\AA\end{array}$} & \multirow[t]{2}{*}{ Notes } \\
\hline $\mathrm{cm}^{-1}$ & & & $J$ & $\mathrm{~cm}^{-1}$ & & & & & \\
\hline 122954.180 & $\left({ }^{3} \mathrm{H}\right) 4 \mathrm{f}$ & $6[9]$ & $19 / 2$ & 103644.800 & $\left({ }^{3} \mathrm{H}\right) 4 \mathrm{~d}^{4} \mathrm{~K}_{17 / 2}$ & 5177.388 & +1.169 & 5177.394 & $\mathrm{~J} 78$, lab, good agreement \\
\hline 122952.730 & $\left({ }^{3} \mathrm{H}\right) 4 \mathrm{f}$ & $6[9]$ & $17 / 2$ & $\begin{array}{l}103644.800 \\
103706.530 \\
103878.370 \\
104119.710\end{array}$ & $\begin{array}{l}\left({ }^{3} \mathrm{H}\right) 4 \mathrm{~d}^{4} \mathrm{~K}_{17 / 2} \\
\left({ }^{3} \mathrm{H}\right) 4 \mathrm{~d}^{4} \mathrm{~K}_{15 / 2} \\
\left({ }^{3} \mathrm{H}\right) 4 \mathrm{~d}^{4} \mathrm{I}_{15 / 2} \\
\left({ }^{3} \mathrm{H}\right) 4 \mathrm{~d}^{2} \mathrm{~K}_{15 / 2}\end{array}$ & $\begin{array}{l}5177.777 \\
5194.384 \\
5241.181 \\
5308.346\end{array}$ & $\begin{array}{l}-0.930 \\
+0.798 \\
+0.558 \\
+0.518\end{array}$ & $\begin{array}{l}5194.387 \\
5241.183 \\
5308.350\end{array}$ & $\begin{array}{l}\text { blend } \\
\text { lab, good agreement } \\
\text { J78, lab, good agreement } \\
\text { J78, lab, good agreement }\end{array}$ \\
\hline 123007.910 & $\left({ }^{3} \mathrm{H}\right) 4 \mathrm{f}$ & $6[8]$ & $17 / 2$ & $\begin{array}{l}103644.800 \\
103706.530 \\
103878.370 \\
104119.710 \\
108337.860\end{array}$ & $\begin{array}{l}\left({ }^{3} \mathrm{H}\right) 4 \mathrm{~d}^{4} \mathrm{~K}_{17 / 2} \\
\left({ }^{3} \mathrm{H}\right) 4 \mathrm{~d}^{4} \mathrm{~K}_{15 / 2} \\
\left({ }^{3} \mathrm{H}\right) 4 \mathrm{~d}^{4} \mathrm{I}_{15 / 2} \\
\left({ }^{3} \mathrm{H}\right) 4 \mathrm{~d}^{2} \mathrm{~K}_{15 / 2} \\
\left({ }^{3} \mathrm{G}\right) 4 \mathrm{~d}^{4} \mathrm{I}_{15 / 2}\end{array}$ & $\begin{array}{l}5163.021 \\
5179.534 \\
5226.062 \\
5292.838 \\
6814.729\end{array}$ & $\begin{array}{l}+0.498 \\
+0.534 \\
+0.820 \\
-1.419 \\
-1.183\end{array}$ & $\begin{array}{l}5163.018 \\
5179.540 \\
5226.070\end{array}$ & $\begin{array}{l}\text { J78, lab, good agreement } \\
\text { J78, lab, good agreement } \\
\text { lab, good agreement } \\
\text { at the noise level }\end{array}$ \\
\hline 122910.920 & $\left({ }^{3} \mathrm{H}\right) 4 \mathrm{f}$ & $6[8]$ & $15 / 2$ & $\begin{array}{l}103706.530 \\
103832.050 \\
103878.370 \\
104064.670 \\
104119.710 \\
104315.370 \\
104622.300 \\
108463.910 \\
108648.695 \\
109049.600\end{array}$ & $\begin{array}{l}\left({ }^{3} \mathrm{H}\right) 4 d^{4} \mathrm{~K}_{15 / 2} \\
\left({ }^{3} \mathrm{H}\right) 4 \mathrm{~d}^{4} \mathrm{~K}_{13 / 2} \\
\left({ }^{3} \mathrm{H}\right) 4 \mathrm{~d}^{4} \mathrm{I}_{15 / 2} \\
\left({ }^{3} \mathrm{H}\right) 4 \mathrm{~d}^{4} \mathrm{I}_{13 / 2} \\
\left({ }^{3} \mathrm{H}\right) 4 \mathrm{~d}^{2} \mathrm{~K}_{15 / 2} \\
\left({ }^{3} \mathrm{H}\right) 4 \mathrm{~d}^{2} \mathrm{~K}_{13 / 2} \\
\left({ }^{3} \mathrm{H}\right) 4 \mathrm{~d}^{2} \mathrm{I}_{13 / 2} \\
\left({ }^{3} \mathrm{G}\right) 4 \mathrm{~d}^{4} \mathrm{I}_{13 / 2} \\
\left({ }^{1} \mathrm{I}\right) 5 \mathrm{~s} \mathrm{e}^{2} \mathrm{I}_{13 / 2} \\
\left({ }^{3} \mathrm{G}\right) 4 \mathrm{~d}^{2} \mathrm{I}_{13 / 2}\end{array}$ & $\begin{array}{l}5205.693 \\
5239.942 \\
5252.695 \\
5304.620 \\
5320.157 \\
5376.136 \\
5466.362 \\
6919.939 \\
7009.596 \\
7212.332\end{array}$ & $\begin{array}{l}-0.207 \\
+0.015 \\
-0.107 \\
-0.357 \\
+0.082 \\
+0.132 \\
+0.698 \\
-0.887 \\
-1.436 \\
-1.456\end{array}$ & $\begin{array}{l}5205.70 \\
5239.948 \\
5252.702 \\
5304.60 \\
5320.18 \\
5376.12 \\
5466.38 \\
7009.6 ? \\
7212.33 ?\end{array}$ & $\begin{array}{l}\text { blend } \\
\text { J78, lab, computed too weak } \\
\text { lab, computed too weak } \\
\text { lab, computed too weak } \\
\text { lab, good agreement } \\
\text { lab, computed too weak } \\
\text { good agreement } \\
\text { at the continuum level } \\
\text { computed too weak? } \\
\text { computed too weak? }\end{array}$ \\
\hline 123018.430 & $\left({ }^{3} \mathrm{H}\right) 4 \mathrm{f}$ & $6[7]$ & $15 / 2$ & $\begin{array}{l}103617.580 \\
103644.800 \\
103706.530 \\
103832.050 \\
103878.370 \\
104064.670 \\
104119.710 \\
104622.300 \\
108337.860\end{array}$ & $\begin{array}{l}\left({ }^{3} \mathrm{H}\right) 4 d^{4} \mathrm{H}_{13 / 2} \\
\left({ }^{3} \mathrm{H}\right) 4 \mathrm{~d}^{4} \mathrm{~K}_{17 / 2} \\
\left({ }^{3} \mathrm{H}\right) 4 \mathrm{~d}^{4} \mathrm{~K}_{15 / 2} \\
\left({ }^{3} \mathrm{H}\right) 4 \mathrm{~d}^{4} \mathrm{~K}_{13 / 2} \\
\left({ }^{3} \mathrm{H}\right) 4 \mathrm{~d}^{4} \mathrm{I}_{15 / 2} \\
\left({ }^{3} \mathrm{H}\right) 4 \mathrm{~d}^{4} \mathrm{I}_{13 / 2} \\
\left({ }^{3} \mathrm{H}\right) 4 \mathrm{~d}^{2} \mathrm{~K}_{15 / 2} \\
\left({ }^{3} \mathrm{H}\right) 4 \mathrm{~d}^{2} \mathrm{I}_{13 / 2} \\
\left({ }^{3} \mathrm{G}\right) 4 \mathrm{~d}^{4} \mathrm{I}_{15 / 2}\end{array}$ & $\begin{array}{l}5152.978 \\
5160.218 \\
5176.713 \\
5210.580 \\
5223.190 \\
5274.530 \\
5289.892 \\
5434.415 \\
6809.845\end{array}$ & $\begin{array}{l}+0.761 \\
-0.354 \\
+0.364 \\
-1.104 \\
+0.447 \\
-1.138 \\
-0.894 \\
-1.378 \\
-1.228\end{array}$ & $\begin{array}{l}5152.985 \\
5160.213 \\
5176.722 \\
5210.65 ? \\
5223.25 \\
5274.53 \\
5289.899\end{array}$ & $\begin{array}{l}\text { lab, good agreement } \\
\text { lab, good agreement } \\
\text { J78,lab, good agreement } \\
\text { computed too weak? } \\
\text { blend, good agreement } \\
\text { good agreement } \\
\text { lab, good agreement } \\
\text { at the noise level } \\
\text { at the noise level }\end{array}$ \\
\hline 123015.400 & $\left({ }^{3} \mathrm{H}\right) 4 \mathrm{f}$ & $6[7]$ & $13 / 2$ & $\begin{array}{l}103600.430 \\
103617.580 \\
103706.530 \\
103751.660 \\
103878.370 \\
104119.710 \\
104765.450 \\
105063.550 \\
105288.850 \\
106045.690 \\
108181.550\end{array}$ & $\begin{array}{l}\left({ }^{3} \mathrm{H}\right) 4 d^{4} \mathrm{G}_{11 / 2} \\
\left({ }^{3} \mathrm{H}\right) 4 \mathrm{~d}^{4} \mathrm{H}_{13 / 2} \\
\left({ }^{3} \mathrm{H}\right) 4 \mathrm{~d}^{4} \mathrm{~K}_{15 / 2} \\
\left({ }^{3} \mathrm{H}\right) 4 \mathrm{~d}^{4} \mathrm{H}_{11 / 2} \\
\left({ }^{3} \mathrm{H}\right) 4 \mathrm{~d}^{4} \mathrm{I}_{15 / 2} \\
\left({ }^{3} \mathrm{H}\right) 4 \mathrm{~d}^{2} \mathrm{~K}_{15 / 2} \\
\left({ }^{3} \mathrm{H}\right) 4 \mathrm{~d}^{2} \mathrm{I}_{11 / 2} \\
\left({ }^{3} \mathrm{~F}\right) 4 \mathrm{~d}^{4} \mathrm{G}_{11 / 2} \\
\left({ }^{3} \mathrm{~F}\right) 4 \mathrm{~d}^{4} \mathrm{H}_{13 / 2} \\
\left({ }^{3} \mathrm{H}\right) 4 \mathrm{~d}^{2} \mathrm{H}_{11 / 2} \\
\left({ }^{3} \mathrm{G}\right) 4 \mathrm{~d}^{4} \mathrm{G}_{11 / 2}\end{array}$ & $\begin{array}{l}5149.230 \\
5153.783 \\
5177.525 \\
5189.655 \\
5224.017 \\
5290.740 \\
5477.945 \\
5568.910 \\
5639.690 \\
5891.220 \\
6739.478\end{array}$ & $\begin{array}{l}+0.424 \\
+0.761 \\
-0.341 \\
-0.783 \\
-0.132 \\
-1.258 \\
-1.275 \\
-1.164 \\
-1.357 \\
-1.302 \\
-1.459\end{array}$ & $\begin{array}{l}5224.025 \\
5290.730 \\
5477.95 \\
5568.92\end{array}$ & $\begin{array}{l}\text { lab, good agreement } \\
\text { lab, good agreement } \\
\text { blend } \\
\text { blend, good agreement } \\
\text { lab, good agreement } \\
\text { computed too weak } \\
\text { good agreement } \\
\text { good agreement } \\
\text { blend } \\
\text { blend } \\
\text { at the noise level }\end{array}$ \\
\hline 122990.620 & $\left({ }^{3} \mathrm{H}\right) 4 \mathrm{f}$ & $6[6]$ & $13 / 2$ & $\begin{array}{l}103706.530 \\
103751.660 \\
103832.050 \\
103878.370 \\
103973.780 \\
104064.670 \\
104119.710 \\
104174.270 \\
104315.370 \\
104622.300 \\
104765.450\end{array}$ & $\begin{array}{l}\left({ }^{3} \mathrm{H}\right) 4 d^{4} \mathrm{~K}_{15 / 2} \\
\left({ }^{3} \mathrm{H}\right) 4 \mathrm{~d}^{4} \mathrm{H}_{11 / 2} \\
\left({ }^{3} \mathrm{H}\right) 4 \mathrm{~d}^{4} \mathrm{~K}_{13 / 2} \\
\left({ }^{3} \mathrm{H}\right) 4 \mathrm{~d}^{4} \mathrm{I}_{15 / 2} \\
\left({ }^{3} \mathrm{H}\right) 4 \mathrm{~d}^{4} \mathrm{~K}_{11 / 2} \\
\left({ }^{3} \mathrm{H}\right) 4 \mathrm{~d}^{4} \mathrm{I}_{13 / 2} \\
\left({ }^{3} \mathrm{H}\right) 4 \mathrm{~d}^{2} \mathrm{~K}_{15 / 2} \\
\left({ }^{3} \mathrm{H}\right) 4 \mathrm{~d}^{4} \mathrm{I}_{11 / 2} \\
\left({ }^{3} \mathrm{H}\right) 4 \mathrm{~d}^{2} \mathrm{~K}_{13 / 2} \\
\left({ }^{3} \mathrm{H}\right) 4 \mathrm{~d}^{2} \mathrm{I}_{13 / 2} \\
\left({ }^{3} \mathrm{H}\right) 4 \mathrm{~d}^{2} \mathrm{I}_{11 / 2}\end{array}$ & $\begin{array}{l}5184.178 \\
5196.339 \\
5218.143 \\
5230.790 \\
5257.034 \\
5282.281 \\
5297.687 \\
5313.049 \\
5353.192 \\
5442.643 \\
5485.393\end{array}$ & $\begin{array}{l}-0.976 \\
-0.126 \\
-0.028 \\
-1.208 \\
-0.940 \\
-1.039 \\
-1.010 \\
-0.954 \\
+0.205 \\
+0.049 \\
+0.141\end{array}$ & $\begin{array}{l}5196.32 \\
5218.149 \\
5230.80 \\
\\
5282.29 \\
5297.7 \\
5353.22 \\
5442.65 \\
5485.40\end{array}$ & $\begin{array}{l}\text { blend } \\
\text { computed too weak } \\
\text { lab, good agreement } \\
\text { good agreement } \\
\text { blend } \\
\text { blend,computed too weak } \\
\text { blend } \\
\text { blend } \\
\text { blend, computed too strong } \\
\text { J78, lab, good agreement } \\
\text { computed too strong }\end{array}$ \\
\hline
\end{tabular}


Table 7. continued.

\begin{tabular}{|c|c|c|c|c|c|c|c|c|c|}
\hline \multicolumn{4}{|c|}{ Upper level } & \multicolumn{2}{|c|}{ Lower level } & \multirow{2}{*}{$\begin{array}{c}\lambda(\text { calc }) \\
\AA\end{array}$} & \multirow{2}{*}{$\begin{array}{c}\log g f \\
\text { KUR }\end{array}$} & \multirow{2}{*}{$\begin{array}{c}\lambda(\mathrm{obs}) \\
\AA\end{array}$} & \multirow[t]{2}{*}{ Notes } \\
\hline $\mathrm{cm}^{-1}$ & & & $J$ & $\mathrm{~cm}^{-1}$ & & & & & \\
\hline 122990.620 & cont. & & & $\begin{array}{l}105063.550 \\
105763.270 \\
106045.690 \\
108630.429 \\
109049.600 \\
109389.880 \\
109683.280\end{array}$ & $\begin{array}{l}\left({ }^{3} \mathrm{~F}\right) 4 d^{4} \mathrm{G}_{11 / 2} \\
\left({ }^{3} \mathrm{~F}\right) 4 d^{2} \mathrm{H}_{11 / 2} \\
\left({ }^{3} \mathrm{H}\right) 4 \mathrm{~d}^{2} \mathrm{H}_{11 / 2} \\
\left({ }^{1} \mathrm{I}\right) 5 \mathrm{~s} \mathrm{e}^{2} \mathrm{I}_{11 / 2} \\
\left({ }^{3} \mathrm{G}\right) 4 \mathrm{~d}^{2} \mathrm{I}_{13 / 2} \\
\left({ }^{3} \mathrm{G}\right) 4 \mathrm{~d}^{2} \mathrm{I}_{11 / 2} \\
\left({ }^{3} \mathrm{G}\right) 4 \mathrm{~d}^{2} \mathrm{H}_{11 / 2}\end{array}$ & $\begin{array}{l}5576.608 \\
5803.114 \\
5899.835 \\
6961.775 \\
7171.100 \\
7350.516 \\
7512.581\end{array}$ & $\begin{array}{l}-0.487 \\
-0.380 \\
+0.277 \\
-1.168 \\
-1.477 \\
-1.297 \\
-0.706\end{array}$ & $\begin{array}{l}5576.60 \\
5803.12 \\
5899.82 \\
\\
7350.49 ?\end{array}$ & $\begin{array}{l}\text { computed too strong } \\
\text { computed too weak } \\
\text { good agreement } \\
\text { at the continuum level } \\
\text { at the continuum level } \\
\text { computed too weak? } \\
\text { blend, computed too weak? }\end{array}$ \\
\hline 123037.430 & $\left({ }^{3} \mathrm{H}\right) 4 \mathrm{f}$ & $6[6]$ & $11 / 2$ & $\begin{array}{l}103751.660 \\
103771.320 \\
103832.050 \\
103874.260 \\
104064.670 \\
104315.370 \\
104622.300 \\
104765.450 \\
104807.210 \\
104916.550 \\
105063.550 \\
105398.850 \\
105763.270 \\
106045.690 \\
106097.520 \\
106924.430 \\
109683.280\end{array}$ & $\begin{array}{l}\left({ }^{3} \mathrm{H}\right) 4 \mathrm{~d}^{4} \mathrm{H}_{11 / 2} \\
\left({ }^{3} \mathrm{H}\right) 4 \mathrm{~d}^{4} \mathrm{G}_{9 / 2} \\
\left({ }^{3} \mathrm{H}\right) 4 \mathrm{~d}^{4} \mathrm{~K}_{13 / 2} \\
\left({ }^{3} \mathrm{H}\right) 4 \mathrm{~d}^{4} \mathrm{H}_{9 / 2} \\
\left({ }^{3} \mathrm{H}\right) 4 \mathrm{~d}^{4} \mathrm{I}_{13 / 2} \\
\left({ }^{3} \mathrm{H}\right) 4 \mathrm{~d}^{2} \mathrm{~K}_{13 / 2} \\
\left({ }^{3} \mathrm{H}\right) 4 \mathrm{~d}^{2} \mathrm{I}_{13 / 2} \\
\left({ }^{3} \mathrm{H}\right) 4 \mathrm{~d}^{2} \mathrm{I}_{11 / 2} \\
\left({ }^{3} \mathrm{H}\right) 4 \mathrm{~d}^{2} \mathrm{G}_{9 / 2} \\
\left({ }^{3} \mathrm{H}\right) 4 \mathrm{~d}^{4} \mathrm{~F}_{9 / 2} \\
\left({ }^{3} \mathrm{~F}\right) 4 \mathrm{~d}^{4} \mathrm{G}_{11 / 2} \\
\left({ }^{3} \mathrm{~F}\right) 4 \mathrm{~d}^{4} \mathrm{H}_{11 / 2} \\
\left({ }^{3} \mathrm{~F}\right) 4 \mathrm{~d}^{2} \mathrm{H}_{11 / 2} \\
\left({ }^{3} \mathrm{H}\right) 4 \mathrm{~d}^{2} \mathrm{H}_{11 / 2} \\
\left({ }^{3} \mathrm{H}\right) 4 \mathrm{~d}^{2} \mathrm{H}_{9 / 2} \\
\left({ }^{3} \mathrm{~F}\right) 4 \mathrm{~d}^{2} \mathrm{G}_{9 / 2} \\
\left({ }^{3} \mathrm{G}\right) 4 \mathrm{~d}^{2} \mathrm{H}_{11 / 2}\end{array}$ & $\begin{array}{l}5183.727 \\
5189.016 \\
5205.425 \\
5216.891 \\
5269.248 \\
5339.807 \\
5428.808 \\
5471.340 \\
5483.874 \\
5516.963 \\
5562.084 \\
5667.818 \\
5787.389 \\
5883.582 \\
5901.584 \\
6204.452 \\
7486.247\end{array}$ & $\begin{array}{l}+0.242 \\
-0.187 \\
-0.558 \\
-0.503 \\
-0.797 \\
-0.759 \\
-0.405 \\
-0.934 \\
-0.019 \\
-0.234 \\
-1.223 \\
-1.176 \\
-0.146 \\
+0.287 \\
-0.581 \\
-1.391 \\
-0.596\end{array}$ & $\begin{array}{l}5183.713 \\
5189.013 \\
5205.427 \\
\\
5269.235 \\
5428.80 \\
5483.85\end{array}$ & $\begin{array}{l}\text { J78, lab, blend } \\
\text { lab } \\
\text { lab, blend } \\
\text { blend } \\
\text { lab } \\
\text { lab } \\
\text { wrong, not obs }\end{array}$ \\
\hline 123002.288 & $\left({ }^{3} \mathrm{H}\right) 4 \mathrm{f}$ & $6[5]$ & $11 / 2$ & $\begin{array}{l}103165.320 \\
103600.430 \\
103617.580 \\
103683.070 \\
103751.660 \\
103771.320 \\
104765.450 \\
104807.210 \\
104916.550 \\
105063.550 \\
106045.690 \\
106722.170 \\
108181.550 \\
109811.920\end{array}$ & $\begin{array}{l}\left({ }^{3} \mathrm{P}\right) 4 d^{4} \mathrm{~F}_{9 / 2} \\
\left({ }^{3} \mathrm{H}\right) 4 d^{4} \mathrm{G}_{11 / 2} \\
\left({ }^{3} \mathrm{H}\right) 4 \mathrm{~d}^{4} \mathrm{H}_{13 / 2} \\
\left({ }^{3} \mathrm{H}\right) 4 \mathrm{~d}^{4} \mathrm{~F}_{9 / 2} \\
\left({ }^{3} \mathrm{H}\right) 4 \mathrm{~d}^{4} \mathrm{H}_{11 / 2} \\
\left({ }^{3} \mathrm{H}\right) 4 \mathrm{~d}^{4} \mathrm{G}_{9 / 2} \\
\left({ }^{3} \mathrm{H}\right) 4 \mathrm{~d}^{2} \mathrm{I}_{11 / 2} \\
\left({ }^{3} \mathrm{H}\right) 4 \mathrm{~d}^{2} \mathrm{G}_{9 / 2} \\
\left({ }^{3} \mathrm{H}\right) 4 \mathrm{~d}^{4} \mathrm{~F}_{9 / 2} \\
\left({ }^{3} \mathrm{~F}\right) 4 \mathrm{~d}^{4} \mathrm{G}_{11 / 2} \\
\left({ }^{3} \mathrm{H}\right) 4 \mathrm{~d}^{2} \mathrm{H}_{11 / 2} \\
\left({ }^{3} \mathrm{~F}\right) 4 d^{4} \mathrm{~F}_{9 / 2} \\
\left({ }^{3} \mathrm{G}\right) 4 d^{4} \mathrm{G}_{11 / 2} \\
\left({ }^{3} \mathrm{G}\right) 4 d^{4} \mathrm{~F}_{9 / 2}\end{array}$ & $\begin{array}{l}5039.690 \\
5152.712 \\
5157.271 \\
5174.754 \\
5193.192 \\
5198.501 \\
5481.886 \\
5494.468 \\
5527.686 \\
5572.983 \\
5895.778 \\
6140.765 \\
6745.444 \\
7579.208\end{array}$ & $\begin{array}{l}-0.526 \\
+0.662 \\
+0.380 \\
-0.491 \\
-0.719 \\
-1.338 \\
-1.256 \\
-0.835 \\
-1.221 \\
-0.697 \\
-1.407 \\
-0.940 \\
-1.310 \\
-1.201\end{array}$ & $\begin{array}{l}5152.70 \\
\\
5174.75 \\
5193.191\end{array}$ & $\begin{array}{l}\text { lab } \\
\text { blend } \\
\text { lab } \\
\text { blend }\end{array}$ \\
\hline 123026.350 & $\left.{ }^{3} \mathrm{H}\right) 4 \mathrm{f}$ & $6[5]$ & $9 / 2$ & $\begin{array}{l}103102.860 \\
103751.660 \\
103771.320 \\
103874.260 \\
104107.950 \\
104481.590 \\
104807.210 \\
104916.550 \\
104993.860 \\
105763.270 \\
106045.690 \\
106097.520 \\
106722.170 \\
106767.210 \\
106924.430 \\
109683.280\end{array}$ & $\begin{array}{l}\left({ }^{3} \mathrm{P}\right) 4 \mathrm{~d}^{4} \mathrm{D}_{7 / 2} \\
\left({ }^{3} \mathrm{H}\right) 4 \mathrm{~d}^{4} \mathrm{H}_{11 / 2} \\
\left({ }^{3} \mathrm{H}\right) 4 \mathrm{~d}^{4} \mathrm{G}_{9 / 2} \\
\left({ }^{3} \mathrm{H}\right) 4 \mathrm{~d}^{4} \mathrm{H}_{9 / 2} \\
\left({ }^{3} \mathrm{P}\right) 4 \mathrm{~d}^{4} \mathrm{~F}_{7 / 2} \\
\left({ }^{3} \mathrm{H}\right) 4 \mathrm{~d}^{2} \mathrm{~F}_{7 / 2} \\
\left({ }^{3} \mathrm{H}\right) 4 \mathrm{~d}^{2} \mathrm{G}_{9 / 2} \\
\left({ }^{3} \mathrm{H}\right) 4 \mathrm{~d}^{4} \mathrm{~F}_{9 / 2} \\
\left({ }^{3} \mathrm{~F}\right) 4 \mathrm{~d}^{4} \mathrm{D}_{7 / 2} \\
\left({ }^{3} \mathrm{~F}\right) 4 \mathrm{~d}^{2} \mathrm{H}_{11 / 2} \\
\left({ }^{3} \mathrm{H}\right) 4 \mathrm{~d}^{2} \mathrm{H}_{11 / 2} \\
\left({ }^{3} \mathrm{H}\right) 4 \mathrm{~d}^{2} \mathrm{H}_{9 / 2} \\
\left({ }^{3} \mathrm{~F}\right) 4 \mathrm{~d}^{4} \mathrm{~F}_{9 / 2} \\
\left({ }^{3} \mathrm{~F}\right) 4 \mathrm{~d}^{4} \mathrm{~F}_{7 / 2} \\
\left({ }^{3} \mathrm{~F}\right) 4 \mathrm{~d}^{2} \mathrm{G}_{9 / 2} \\
\left({ }^{3} \mathrm{G}\right) 4 \mathrm{~d}^{2} \mathrm{H}_{11 / 2}\end{array}$ & $\begin{array}{l}5017.801 \\
5186.706 \\
5192.002 \\
5219.909 \\
5284.389 \\
5390.860 \\
5487.209 \\
5520.339 \\
5544.006 \\
5791.103 \\
5887.421 \\
5905.446 \\
6131.699 \\
6148.685 \\
6208.722 \\
7492.464\end{array}$ & $\begin{array}{l}-1.092 \\
-0.152 \\
+0.073 \\
-0.488 \\
-0.355 \\
-1.184 \\
+0.186 \\
-0.063 \\
-1.091 \\
-0.522 \\
-0.109 \\
-0.710 \\
-1.253 \\
-1.351 \\
-0.916 \\
-1.002\end{array}$ & $\begin{array}{l}5186.722 \\
5192.010 \\
\\
5487.21 \\
\\
5791.05 \\
5887.42\end{array}$ & $\begin{array}{l}\text { lab } \\
\text { lab } \\
\text { blend } \\
\text { lab } \\
\text { wrong, not observed }\end{array}$ \\
\hline
\end{tabular}


F. Castelli and R. L. Kurucz: New Fe II energy levels from stellar spectra

Table 7. continued.

\begin{tabular}{|c|c|c|c|c|c|c|c|c|c|}
\hline \multicolumn{4}{|c|}{ Upper level } & \multicolumn{2}{|c|}{ Lower level } & \multirow{2}{*}{$\begin{array}{c}\lambda(\text { calc }) \\
\AA \\
\end{array}$} & \multirow{2}{*}{$\begin{array}{c}\log g f \\
\text { KUR }\end{array}$} & \multirow{2}{*}{$\begin{array}{c}\lambda(\text { obs }) \\
\AA\end{array}$} & \multirow[t]{2}{*}{ Notes } \\
\hline $\mathrm{cm}^{-1}$ & & & $J$ & $\mathrm{~cm}^{-1}$ & & & & & \\
\hline 122988.215 & $\left({ }^{3} \mathrm{H}\right) 4 \mathrm{f}$ & $6[4]$ & $9 / 2$ & $\begin{array}{l}103165.320 \\
103600.430 \\
103683.070 \\
103751.660 \\
103771.320 \\
104765.450 \\
104807.210 \\
104916.550 \\
105063.550 \\
106045.690 \\
106722.170 \\
106924.430 \\
108181.550 \\
109811.920\end{array}$ & $\begin{array}{l}\left({ }^{3} \mathrm{P}\right) 4 \mathrm{~d}^{4} \mathrm{~F}_{9 / 2} \\
\left({ }^{3} \mathrm{H}\right) 4 \mathrm{~d}^{4} \mathrm{G}_{11 / 2} \\
\left({ }^{3} \mathrm{H}\right) 4 \mathrm{~d}^{4} \mathrm{~F}_{9 / 2} \\
\left({ }^{3} \mathrm{H}\right) 4 \mathrm{~d}^{4} \mathrm{H}_{11 / 2} \\
\left({ }^{3} \mathrm{H}\right) 4 \mathrm{~d}^{4} \mathrm{G}_{9 / 2} \\
\left({ }^{3} \mathrm{H}\right) 4 \mathrm{~d}^{2} \mathrm{I}_{11 / 2} \\
\left({ }^{3} \mathrm{H}\right) 4 \mathrm{~d}^{2} \mathrm{G}_{9 / 2} \\
\left({ }^{3} \mathrm{H}\right) 4 \mathrm{~d}^{4} \mathrm{~F}_{9 / 2} \\
\left({ }^{3} \mathrm{~F}\right) 4 \mathrm{~d}^{4} \mathrm{G}_{11 / 2} \\
\left({ }^{3} \mathrm{H}\right) 4 \mathrm{~d}^{2} \mathrm{H}_{11 / 2} \\
\left({ }^{3} \mathrm{~F}\right) 4 \mathrm{~d}^{4} \mathrm{~F}_{9 / 2} \\
\left({ }^{3} \mathrm{~F}\right) 4 \mathrm{~d}^{2} \mathrm{G}_{9 / 2} \\
\left({ }^{3} \mathrm{G}\right) 4 \mathrm{~d}^{4} \mathrm{G}_{11 / 2} \\
\left.{ }^{3} \mathrm{G}\right) 4 \mathrm{~d}^{4} \mathrm{~F}_{9 / 2}\end{array}$ & $\begin{array}{l}5043.266 \\
5156.450 \\
5178.524 \\
5196.989 \\
5202.306 \\
5486.117 \\
5498.718 \\
5531.988 \\
5577.356 \\
5900.673 \\
6146.075 \\
6223.461 \\
6751.852 \\
7587.298 \\
\end{array}$ & $\begin{array}{l}-0.030 \\
+0.529 \\
-0.018 \\
-0.773 \\
-0.787 \\
-1.286 \\
-0.382 \\
-1.028 \\
-0.785 \\
-1.342 \\
-0.412 \\
-1.178 \\
-1.421 \\
-0.695\end{array}$ & $\begin{array}{l}5498.72 \\
5577.35 \\
6146.08\end{array}$ & $\begin{array}{l}\text { lab } \\
\text { lab }\end{array}$ \\
\hline 122980.408 & $\left({ }^{3} \mathrm{H}\right) 4 \mathrm{f}$ & $6[4]$ & $7 / 2$ & $\begin{array}{l}103102.860 \\
103165.320 \\
103683.070 \\
103771.320 \\
103874.260 \\
103921.630 \\
104107.950 \\
104481.590 \\
104807.210 \\
104916.550 \\
104993.860 \\
106097.520 \\
106722.170 \\
106767.210 \\
106924.430\end{array}$ & $\begin{array}{l}\left({ }^{3} \mathrm{P}\right) 4 \mathrm{~d}^{4} \mathrm{D}_{7 / 2} \\
\left({ }^{3} \mathrm{P}\right) 4 \mathrm{~d}^{4} \mathrm{~F}_{9 / 2} \\
\left({ }^{3} \mathrm{H}\right) 4 \mathrm{~d}^{4} \mathrm{~F}_{9 / 2} \\
\left({ }^{3} \mathrm{H}\right) 4 \mathrm{~d}^{4} \mathrm{G}_{9 / 2} \\
\left({ }^{3} \mathrm{H}\right) 4 \mathrm{~d}^{4} \mathrm{H}_{9 / 2} \\
\left({ }^{3} \mathrm{H}\right) 4 \mathrm{~d}^{4} \mathrm{G}_{7 / 2} \\
\left({ }^{3} \mathrm{P}\right) 4 \mathrm{~d}^{4} \mathrm{~F}_{7 / 2} \\
\left({ }^{3} \mathrm{H}\right) 4 \mathrm{~d}^{2} \mathrm{~F}_{7 / 2} \\
\left({ }^{3} \mathrm{H}\right) 4 \mathrm{~d}^{2} \mathrm{G}_{9 / 2} \\
\left({ }^{3} \mathrm{H}\right) 4 \mathrm{~d}^{4} \mathrm{~F}_{9 / 2} \\
\left({ }^{3} \mathrm{~F}\right) 4 \mathrm{~d}^{4} \mathrm{D}_{7 / 2} \\
\left({ }^{3} \mathrm{H}\right) 4 \mathrm{~d}^{2} \mathrm{H}_{9 / 2} \\
\left({ }^{3} \mathrm{~F}\right) 4 \mathrm{~d}^{4} \mathrm{~F}_{9 / 2} \\
\left({ }^{3} \mathrm{~F}\right) 4 d^{4} \mathrm{~F}_{7 / 2} \\
\left({ }^{3} \mathrm{~F}\right) 4 d^{2} \mathrm{G}_{9 / 2}\end{array}$ & $\begin{array}{l}5029.399 \\
5045.253 \\
5180.619 \\
5204.420 \\
5232.461 \\
5245.466 \\
5297.253 \\
5404.248 \\
5501.081 \\
5534.379 \\
5558.167 \\
5921.516 \\
6149.026 \\
6166.108 \\
6226.487\end{array}$ & $\begin{array}{l}-0.735 \\
-0.962 \\
-1.116 \\
-0.034 \\
-0.656 \\
-1.235 \\
+0.049 \\
-0.598 \\
-0.147 \\
-0.071 \\
-0.731 \\
-0.986 \\
-0.728 \\
-1.069 \\
-1.380\end{array}$ & $\begin{array}{l}5204.419 \\
5297.26\end{array}$ & \\
\hline 122946.419 & $\left({ }^{3} \mathrm{H}\right) 4 \mathrm{f}$ & $6[3]$ & $7 / 2$ & $\begin{array}{l}103102.860 \\
103165.320 \\
103683.070 \\
103771.320 \\
104107.950 \\
104807.210 \\
105155.090 \\
105211.062 \\
106097.520 \\
106722.170 \\
106924.430 \\
109811.920\end{array}$ & $\begin{array}{l}\left({ }^{3} \mathrm{P}\right) 4 \mathrm{~d}^{4} \mathrm{D}_{7 / 2} \\
\left({ }^{3} \mathrm{P}\right) 4 \mathrm{~d}^{4} \mathrm{~F}_{9 / 2} \\
\left({ }^{3} \mathrm{H}\right) 4 \mathrm{~d}^{4} \mathrm{~F}_{9 / 2} \\
\left({ }^{3} \mathrm{H}\right) 4 \mathrm{~d}^{4} \mathrm{G}_{9 / 2} \\
\left({ }^{3} \mathrm{P}\right) 4 \mathrm{~d}^{4} \mathrm{~F}_{7 / 2} \\
\left({ }^{3} \mathrm{H}\right) 4 \mathrm{~d}^{2} \mathrm{G}_{9 / 2} \\
\left({ }^{3} \mathrm{~F}\right) 4 \mathrm{~d}^{4} \mathrm{G}_{9 / 2} \\
\left({ }^{5} \mathrm{D}\right) 5 \mathrm{~d}^{4} \mathrm{G}_{9 / 2} \\
\left({ }^{3} \mathrm{H}\right) 4 \mathrm{~d}^{2} \mathrm{H}_{9 / 2} \\
\left({ }^{3} \mathrm{~F}\right) 4 \mathrm{~d}^{4} \mathrm{~F}_{9 / 2} \\
\left({ }^{3} \mathrm{~F}\right) 4 \mathrm{~d}^{2} \mathrm{G}_{9 / 2} \\
\left({ }^{3} \mathrm{G}\right) 4 \mathrm{~d}^{4} \mathrm{~F}_{9 / 2}\end{array}$ & $\begin{array}{l}5038.014 \\
5053.922 \\
5189.760 \\
5213.645 \\
5306.811 \\
5511.388 \\
5619.156 \\
5636.890 \\
5933.462 \\
6161.908 \\
6239.696 \\
7611.442\end{array}$ & $\begin{array}{l}-1.413 \\
+0.160 \\
+0.167 \\
-0.746 \\
-0.814 \\
-0.043 \\
-1.229 \\
-1.411 \\
-1.332 \\
-0.227 \\
-0.856 \\
-0.504\end{array}$ & 5511.40 & lab. \\
\hline 123219.200 & $\left({ }^{3} \mathrm{H}\right) 4 \mathrm{f}$ & $5[8]$ & $17 / 2$ & $\begin{array}{l}103644.800 \\
103706.530 \\
103878.370 \\
104119.710\end{array}$ & $\begin{array}{l}\left({ }^{3} \mathrm{H}\right) 4 \mathrm{~d}^{4} \mathrm{~K}_{17 / 2} \\
\left({ }^{3} \mathrm{H}\right) 4 \mathrm{~d}^{4} \mathrm{~K}_{15 / 2} \\
\left({ }^{3} \mathrm{H}\right) 4 \mathrm{~d}^{4} \mathrm{I}_{15 / 2} \\
\left({ }^{3} \mathrm{H}\right) 4 \mathrm{~d}^{2} \mathrm{~K}_{15 / 2}\end{array}$ & $\begin{array}{l}5107.290 \\
5123.448 \\
5168.969 \\
5234.285\end{array}$ & $\begin{array}{l}-0.983 \\
+0.347 \\
+0.064 \\
+0.991\end{array}$ & $\begin{array}{l}5123.45 \\
5234.283\end{array}$ & $\begin{array}{l}\text { lab } \\
\text { blend } \\
\text { lab }\end{array}$ \\
\hline 123193.090 & $\left({ }^{3} \mathrm{H}\right) 4 \mathrm{f}$ & $5[8]$ & $15 / 2$ & $\begin{array}{l}103706.530 \\
103832.050 \\
103878.370 \\
104064.670 \\
104119.710 \\
104315.370 \\
104622.300\end{array}$ & $\begin{array}{l}\left({ }^{3} \mathrm{H}\right) 4 \mathrm{~d}^{4} \mathrm{~K}_{15 / 2} \\
\left({ }^{3} \mathrm{H}\right) 4 \mathrm{~d}^{4} \mathrm{~K}_{13 / 2} \\
\left({ }^{3} \mathrm{H}\right) 4 \mathrm{~d}^{4} \mathrm{I}_{15 / 2} \\
\left({ }^{3} \mathrm{H}\right) 4 \mathrm{~d}^{4} \mathrm{I}_{13 / 2} \\
\left({ }^{3} \mathrm{H}\right) 4 \mathrm{~d}^{2} \mathrm{~K}_{15 / 2} \\
\left({ }^{3} \mathrm{H}\right) 4 \mathrm{~d}^{2} \mathrm{~K}_{13 / 2} \\
\left({ }^{3} \mathrm{H}\right) 4 \mathrm{~d}^{2} \mathrm{I}_{13 / 2}\end{array}$ & $\begin{array}{l}5130.313 \\
5163.574 \\
5175.957 \\
5226.368 \\
5241.450 \\
5295.776 \\
5383.304\end{array}$ & $\begin{array}{l}-0.507 \\
+0.908 \\
-0.540 \\
-0.216 \\
-0.301 \\
-0.452 \\
+0.146\end{array}$ & $\begin{array}{l}5163.55 \\
5175.95 \\
\\
5241.465 \\
5295.773 \\
5383.32\end{array}$ & $\begin{array}{l}\text { lab } \\
\text { blend } \\
\text { lab } \\
\text { blend }\end{array}$ \\
\hline
\end{tabular}


Table 7. continued.

\begin{tabular}{|c|c|c|c|c|c|c|c|c|c|}
\hline \multicolumn{4}{|c|}{ Upper level } & \multicolumn{2}{|c|}{ Lower level } & \multirow{2}{*}{$\begin{array}{c}\lambda(\text { calc }) \\
\AA\end{array}$} & \multirow{2}{*}{$\begin{array}{c}\log g f \\
\text { KUR }\end{array}$} & \multirow{2}{*}{$\begin{array}{c}\lambda(\mathrm{obs}) \\
\AA\end{array}$} & \multirow[t]{2}{*}{ Notes } \\
\hline $\mathrm{cm}^{-1}$ & & & $J$ & $\mathrm{~cm}^{-1}$ & & & & & \\
\hline 123238.440 & $\left({ }^{3} \mathrm{H}\right) 4 \mathrm{f}$ & $5[7]$ & $15 / 2$ & $\begin{array}{l}103617.580 \\
103706.530 \\
103832.050 \\
103878.370 \\
104064.670 \\
104119.710 \\
104315.370 \\
105288.850\end{array}$ & $\begin{array}{l}\left({ }^{3} \mathrm{H}\right) 4 d^{4} \mathrm{H}_{13 / 2} \\
\left({ }^{3} \mathrm{H}\right) 4 d^{4} \mathrm{~K}_{15 / 2} \\
\left({ }^{3} \mathrm{H}\right) 4 \mathrm{~d}^{4} \mathrm{~K}_{13 / 2} \\
\left({ }^{3} \mathrm{H}\right) 4 \mathrm{~d}^{4} \mathrm{I}_{15 / 2} \\
\left({ }^{3} \mathrm{H}\right) 4 \mathrm{~d}^{4} \mathrm{I}_{13 / 2} \\
\left({ }^{3} \mathrm{H}\right) 4 \mathrm{~d}^{2} \mathrm{~K}_{15 / 2} \\
\left({ }^{3} \mathrm{H}\right) 4 \mathrm{~d}^{2} \mathrm{~K}_{13 / 2} \\
\left({ }^{3} \mathrm{~F}\right) 4 \mathrm{~d}^{4} \mathrm{H}_{13 / 2}\end{array}$ & $\begin{array}{l}5095.196 \\
5118.401 \\
5151.507 \\
5163.831 \\
5214.007 \\
5229.017 \\
5283.085 \\
5569.611\end{array}$ & $\begin{array}{l}-0.836 \\
-0.254 \\
-0.716 \\
-0.599 \\
+0.873 \\
-0.045 \\
+0.323 \\
-1.005\end{array}$ & $\begin{array}{l}5095.19 \\
5118.40 \\
5163.82 \\
5214.99 \\
5229.030 \\
5283.093\end{array}$ & $\begin{array}{l}\text { lab } \\
\text { blend } \\
\text { lab } \\
\text { blend } \\
\text { lab } \\
\text { lab } \\
\text { blend }\end{array}$ \\
\hline 123168.680 & $\left({ }^{3} \mathrm{H}\right) 4 \mathrm{f}$ & $5[7]$ & $13 / 2$ & $\begin{array}{l}103600.430 \\
103706.530 \\
103751.660 \\
103832.050 \\
103973.780 \\
104064.670 \\
104174.270 \\
104315.370 \\
104622.300 \\
104765.450 \\
105063.550 \\
105398.850 \\
105763.270 \\
106045.690 \\
108630.429 \\
109683.280\end{array}$ & $\begin{array}{l}\left({ }^{3} \mathrm{H}\right) 4 \mathrm{~d}^{4} \mathrm{G}_{11 / 2} \\
\left({ }^{3} \mathrm{H}\right) 4 \mathrm{~d}^{4} \mathrm{~K}_{15 / 2} \\
\left({ }^{3} \mathrm{H}\right) 4 \mathrm{~d}^{4} \mathrm{H}_{11 / 2} \\
\left({ }^{3} \mathrm{H}\right) 4 \mathrm{~d}^{4} \mathrm{~K}_{11 / 2} \\
\left({ }^{3} \mathrm{H}\right) 4 \mathrm{~d}^{4} \mathrm{~K}_{11 / 2} \\
\left({ }^{3} \mathrm{H}\right) 4 \mathrm{~d}^{4} \mathrm{I}_{13 / 2} \\
\left({ }^{3} \mathrm{H}\right) 4 \mathrm{~d}^{4} \mathrm{I}_{11 / 2} \\
\left({ }^{3} \mathrm{H}\right) 4 \mathrm{~d}^{2} \mathrm{~K}_{13 / 2} \\
\left({ }^{3} \mathrm{H}\right) 4 \mathrm{~d}^{2} \mathrm{I}_{13 / 2} \\
\left({ }^{3} \mathrm{H}\right) 4 \mathrm{~d}^{2} \mathrm{I}_{11 / 2} \\
\left({ }^{3} \mathrm{~F}\right) 4 \mathrm{~d}^{4} \mathrm{G}_{11 / 2} \\
\left({ }^{3} \mathrm{~F}\right) 4 \mathrm{~d}^{4} \mathrm{H}_{11 / 2} \\
\left({ }^{3} \mathrm{~F}\right) 4 \mathrm{~d}^{2} \mathrm{H}_{11 / 2} \\
\left({ }^{3} \mathrm{H}\right) 4 \mathrm{~d}^{2} \mathrm{H}_{11 / 2} \\
\left({ }^{1} \mathrm{I}\right) 5 \mathrm{~s} \mathrm{e} \mathrm{e}^{2} \mathrm{I}_{11 / 2} \\
\left({ }^{3} \mathrm{G}\right) 4 \mathrm{~d}^{2} \mathrm{H}_{11 / 2}\end{array}$ & $\begin{array}{l}5108.895 \\
5136.747 \\
5148.687 \\
5170.092 \\
5208.267 \\
5233.046 \\
5263.242 \\
5302.633 \\
5390.389 \\
5432.319 \\
5521.763 \\
5625.954 \\
5743.747 \\
5838.483 \\
6876.509 \\
7413.385\end{array}$ & $\begin{array}{l}-1.165 \\
-1.256 \\
+0.010 \\
-1.170 \\
-0.275 \\
+0.138 \\
-0.600 \\
-0.581 \\
+0.010 \\
+0.495 \\
-0.481 \\
-1.425 \\
-0.321 \\
-0.311 \\
-1.228 \\
-0.848\end{array}$ & $\begin{array}{l}5148.7 \\
5208.268 \\
5233.041 \\
\\
5390.38 \\
5432.31 \\
5521.78 \\
5743.75\end{array}$ & $\begin{array}{l}\text { computed too strong } \\
\text { lab } \\
\text { computed too strong }\end{array}$ \\
\hline 123249.650 & $\left({ }^{3} \mathrm{H}\right) 4 \mathrm{f}$ & $5[6]$ & $13 / 2$ & $\begin{array}{l}103600.430 \\
103706.530 \\
103751.660 \\
103832.050 \\
103973.780 \\
104064.670 \\
104119.710 \\
104174.270 \\
104315.370 \\
104622.300 \\
105063.550 \\
105288.850 \\
105763.270 \\
106045.690 \\
109049.600 \\
109683.280\end{array}$ & $\begin{array}{l}\left({ }^{3} \mathrm{H}\right) 4 \mathrm{~d}^{4} \mathrm{G}_{11 / 2} \\
\left({ }^{3} \mathrm{H}\right) 4 \mathrm{~d}^{4} \mathrm{~K}_{15 / 2} \\
\left({ }^{3} \mathrm{H}\right) 4 \mathrm{~d}^{4} \mathrm{H}_{11 / 2} \\
\left({ }^{3} \mathrm{H}\right) 4 \mathrm{~d}^{4} \mathrm{~K}_{13 / 2} \\
\left({ }^{3} \mathrm{H}\right) 4 \mathrm{~d}^{4} \mathrm{~K}_{11 / 2} \\
\left({ }^{3} \mathrm{H}\right) 4 \mathrm{~d}^{4} \mathrm{I}_{13 / 2} \\
\left({ }^{3} \mathrm{H}\right) 4 \mathrm{~d}^{2} \mathrm{~K}_{15 / 2} \\
\left.{ }^{(} \mathrm{H}\right) 4 \mathrm{~d}^{4} \mathrm{I}_{11 / 2} \\
\left({ }^{3} \mathrm{H}\right) 4 \mathrm{~d}^{2} \mathrm{~K}_{13 / 2} \\
\left({ }^{3} \mathrm{H}\right) 4 \mathrm{~d}^{2} \mathrm{I}_{13 / 2} \\
\left({ }^{3} \mathrm{~F}\right) 4 \mathrm{~d}^{4} \mathrm{G}_{11 / 2} \\
\left({ }^{3} \mathrm{~F}\right) 4 \mathrm{~d}^{4} \mathrm{H}_{13 / 2} \\
\left({ }^{3} \mathrm{~F}\right) 4 \mathrm{~d}^{2} \mathrm{H}_{11 / 2} \\
\left({ }^{3} \mathrm{H}\right) 4 \mathrm{~d}^{2} \mathrm{H}_{11 / 2} \\
\left({ }^{3} \mathrm{G}\right) 4 \mathrm{~d}^{2} \mathrm{I}_{13 / 2} \\
\left({ }^{3} \mathrm{G}\right) 4 \mathrm{~d}^{2} \mathrm{H}_{11 / 2}\end{array}$ & $\begin{array}{l}5087.842 \\
5115.465 \\
5127.305 \\
5148.533 \\
5186.389 \\
5210.960 \\
5225.953 \\
5240.901 \\
5279.957 \\
5366.958 \\
5497.178 \\
5566.135 \\
5717.150 \\
5811.004 \\
7040.287 \\
7369.139\end{array}$ & $\begin{array}{l}-0.510 \\
-1.027 \\
+0.392 \\
+0.357 \\
+0.210 \\
-0.403 \\
-0.742 \\
-0.464 \\
-0.647 \\
+0.032 \\
-1.156 \\
-1.260 \\
-0.553 \\
-0.182 \\
-1.496 \\
-1.023\end{array}$ & $\begin{array}{l}5087.85 \\
5127.32 \\
5148.52 \\
5186.396 \\
5210.964 \\
\\
5240.911 \\
\\
5366.95 \\
\\
5717.18 \\
5811.00\end{array}$ & $\begin{array}{l}\text { lab } \\
\text { lab, blend } \\
\text { lab } \\
\text { lab } \\
\text { blend } \\
\text { blend } \\
\text { lab }\end{array}$ \\
\hline 123270.340 & $\left({ }^{3} \mathrm{H}\right) 4 \mathrm{f}$ & $5[6]$ & $11 / 2$ & $\begin{array}{l}103600.430 \\
103683.070 \\
103751.660 \\
103771.320 \\
103832.050 \\
103874.260 \\
103973.780 \\
104064.670 \\
104174.270 \\
104192.480 \\
104315.370 \\
104622.300\end{array}$ & $\begin{array}{l}\left({ }^{3} \mathrm{H}\right) 4 d^{4} \mathrm{G}_{11 / 2} \\
\left({ }^{3} \mathrm{H}\right) 4 d^{4} \mathrm{~F}_{9 / 2} \\
\left({ }^{3} \mathrm{H}\right) 4 \mathrm{~d}^{4} \mathrm{H}_{11 / 2} \\
\left({ }^{3} \mathrm{H}\right) 4 \mathrm{~d}^{4} \mathrm{G}_{9 / 2} \\
\left({ }^{3} \mathrm{H}\right) 4 \mathrm{~d}^{4} \mathrm{~K}_{11 / 2} \\
\left({ }^{3} \mathrm{H}\right) 4 \mathrm{~d}^{4} \mathrm{H}_{9 / 2} \\
\left({ }^{3} \mathrm{H}\right) 4 \mathrm{~d}^{4} \mathrm{~K}_{11 / 2} \\
\left({ }^{3} \mathrm{H}\right) 4 \mathrm{~d}^{4} \mathrm{I}_{13 / 2} \\
\left.{ }^{3} \mathrm{H}\right) 4 \mathrm{~d}^{4} \mathrm{I}_{11 / 2} \\
\left({ }^{3} \mathrm{H}\right) 4 \mathrm{~d}^{4} \mathrm{I}_{9 / 2} \\
\left({ }^{3} \mathrm{H}\right) 4 \mathrm{~d}^{2} \mathrm{~K}_{13 / 2} \\
\left.{ }^{3} \mathrm{H}\right) 4 \mathrm{~d}^{2} \mathrm{I}_{13 / 2}\end{array}$ & $\begin{array}{l}5082.491 \\
5103.934 \\
5121.871 \\
5127.035 \\
5143.054 \\
5154.246 \\
5180.829 \\
5205.347 \\
5235.223 \\
5240.220 \\
5274.195 \\
5361.004\end{array}$ & $\begin{array}{l}-0.827 \\
-1.365 \\
+0.373 \\
-0.542 \\
-0.456 \\
+0.127 \\
-0.529 \\
-0.844 \\
-0.536 \\
-1.229 \\
-1.310 \\
-0.422\end{array}$ & $\begin{array}{l}5121.89 \\
5127.05 \\
5143.05 \\
5154.25 \\
5180.84 \\
5235.225\end{array}$ & $\begin{array}{l}\text { blend } \\
\text { lab } \\
\text { lab } \\
\text { lab }\end{array}$ \\
\hline
\end{tabular}


F. Castelli and R. L. Kurucz: New Fe II energy levels from stellar spectra

Table 7. continued.

\begin{tabular}{|c|c|c|c|c|c|c|c|c|c|}
\hline \multicolumn{4}{|c|}{ Upper level } & \multicolumn{2}{|c|}{ Lower level } & \multirow{2}{*}{$\begin{array}{c}\lambda(\mathrm{calc}) \\
\AA\end{array}$} & \multirow{2}{*}{$\begin{array}{c}\log g f \\
\text { KUR }\end{array}$} & \multirow{2}{*}{$\begin{array}{c}\lambda(\mathrm{obs}) \\
\AA\end{array}$} & \multirow[t]{2}{*}{ Notes } \\
\hline $\mathrm{cm}^{-1}$ & & & $J$ & $\mathrm{~cm}^{-1}$ & & & & & \\
\hline 123270.340 & cont. & & & $\begin{array}{l}104807.210 \\
104916.550 \\
105063.550 \\
105155.090 \\
105763.270 \\
106045.690 \\
106722.170 \\
106924.430 \\
109683.280\end{array}$ & $\begin{array}{l}\left({ }^{3} \mathrm{H}\right) 4 d^{2} \mathrm{G}_{9 / 2} \\
\left({ }^{3} \mathrm{H}\right) 4 \mathrm{~d}^{4} \mathrm{~F}_{9 / 2} \\
\left({ }^{3} \mathrm{~F}\right) 4 \mathrm{~d}^{4} \mathrm{G}_{11 / 2} \\
\left({ }^{3} \mathrm{~F}\right) 4 \mathrm{~d}^{4} \mathrm{G}_{9 / 2} \\
\left({ }^{3} \mathrm{~F}\right) 4 \mathrm{~d}^{2} \mathrm{H}_{11 / 2} \\
\left({ }^{3} \mathrm{H}\right) 4 \mathrm{~d}^{2} \mathrm{H}_{11 / 2} \\
\left({ }^{3} \mathrm{~F}\right) 4 \mathrm{~d}^{4} \mathrm{~F}_{9 / 2} \\
\left({ }^{3} \mathrm{~F}\right) 4 \mathrm{~d}^{2} \mathrm{G}_{9 / 2} \\
\left({ }^{3} \mathrm{G}\right) 4 \mathrm{~d}^{2} \mathrm{H}_{11 / 2}\end{array}$ & $\begin{array}{l}5414.696 \\
5446.953 \\
5490.931 \\
5518.678 \\
5710.394 \\
5804.025 \\
6041.291 \\
6116.045 \\
7357.917\end{array}$ & $\begin{array}{l}-0.589 \\
-0.182 \\
-1.162 \\
-0.927 \\
-0.287 \\
-0.029 \\
-1.018 \\
-1.092 \\
-0.867\end{array}$ & $\begin{array}{l}5414.7 \\
5446.95\end{array}$ & $\begin{array}{l}\text { blend } \\
\text { wrong,not observed }\end{array}$ \\
\hline 123251.470 & $\left({ }^{3} \mathrm{H}\right) 4 \mathrm{f}$ & $5[5]$ & $11 / 2$ & $\begin{array}{l}103751.660 \\
103771.320 \\
103874.260 \\
103973.780 \\
104064.670 \\
104174.270 \\
104192.480 \\
104315.370 \\
104765.450 \\
104807.210 \\
104916.550 \\
105063.550 \\
105155.090 \\
105524.460 \\
106018.640 \\
106045.690 \\
106097.520 \\
106924.430 \\
109625.200 \\
110008.300\end{array}$ & $\begin{array}{l}\left({ }^{3} \mathrm{H}\right) 4 \mathrm{~d}^{4} \mathrm{H}_{11 / 2} \\
\left({ }^{3} \mathrm{H}\right) 4 \mathrm{~d}^{4} \mathrm{G}_{9 / 2} \\
\left({ }^{3} \mathrm{H}\right) 4 \mathrm{~d}^{4} \mathrm{H}_{9 / 2} \\
\left({ }^{3} \mathrm{H}\right) 4 \mathrm{~d}^{4} \mathrm{~K}_{11 / 2} \\
\left({ }^{3} \mathrm{H}\right) 4 \mathrm{~d}^{4} \mathrm{I}_{13 / 2} \\
\left({ }^{3} \mathrm{H}\right) 4 \mathrm{~d}^{4} \mathrm{I}_{11 / 2} \\
\left({ }^{3} \mathrm{H}\right) 4 \mathrm{~d}^{4} \mathrm{I}_{9 / 2} \\
\left({ }^{3} \mathrm{H}\right) 4 \mathrm{~d}^{2} \mathrm{~K}_{13 / 2} \\
\left({ }^{3} \mathrm{H}\right) 4 \mathrm{~d}^{2} \mathrm{I}_{11 / 2} \\
\left({ }^{3} \mathrm{H}\right) 4 \mathrm{~d}^{2} \mathrm{G}_{9 / 2} \\
\left({ }^{3} \mathrm{H}\right) 4 \mathrm{~d}^{4} \mathrm{~F}_{9 / 2} \\
\left({ }^{3} \mathrm{~F}\right) 4 \mathrm{~d}^{4} \mathrm{G}_{11 / 2} \\
\left({ }^{3} \mathrm{~F}\right) 4 \mathrm{~d}^{4} \mathrm{G}_{9 / 2} \\
\left({ }^{3} \mathrm{~F}\right) 4 \mathrm{~d}^{4} \mathrm{H}_{9 / 2} \\
\left({ }^{3} \mathrm{~F}\right) 4 \mathrm{~d}^{2} \mathrm{H}_{9 / 2} \\
\left({ }^{3} \mathrm{H}\right) 4 \mathrm{~d}^{2} \mathrm{H}_{11 / 2} \\
\left({ }^{3} \mathrm{H}\right) 4 \mathrm{~d}^{2} \mathrm{H}_{9 / 2} \\
\left({ }^{3} \mathrm{~F}\right) 4 \mathrm{~d}^{2} \mathrm{G}_{9 / 2} \\
\left({ }^{3} \mathrm{G}\right) 4 \mathrm{~d}^{2} \mathrm{G}_{9 / 2} \\
\left.{ }^{3} \mathrm{G}\right) 4 \mathrm{~d}^{2} \mathrm{H}_{9 / 2}\end{array}$ & $\begin{array}{l}5126.827 \\
5132.001 \\
5159.265 \\
5185.899 \\
5210.466 \\
5240.401 \\
5245.408 \\
5279.449 \\
5407.990 \\
5420.234 \\
5452.558 \\
5496.628 \\
5524.433 \\
5639.544 \\
5801.269 \\
5810.389 \\
5827.945 \\
6123.114 \\
7336.744 \\
7548.984\end{array}$ & $\begin{array}{l}-0.236 \\
+0.078 \\
+0.007 \\
+0.058 \\
-0.583 \\
-0.177 \\
-1.139 \\
-1.308 \\
+0.040 \\
-1.131 \\
-0.967 \\
-0.739 \\
-1.032 \\
-1.347 \\
-0.770 \\
-1.328 \\
-0.015 \\
-0.236 \\
-1.064 \\
-1.185\end{array}$ & $\begin{array}{l}5132.0 \\
5159.29 \\
5185.901 \\
\\
5240.405\end{array}$ & $\begin{array}{l}\text { blend } \\
\text { lab } \\
\text { lab, blend } \\
\text { lab } \\
\text { lab } \\
\text { blend } \\
\text { lab } \\
\text { computed too strong } \\
\text { computed too weak }\end{array}$ \\
\hline 123269.378 & $\left({ }^{3} \mathrm{H}\right) 4 \mathrm{f}$ & $5[5]$ & $9 / 2$ & $\begin{array}{l}103751.660 \\
103771.320 \\
103874.260 \\
103921.630 \\
103973.780 \\
103983.510 \\
103986.330 \\
104107.950 \\
104174.270 \\
104765.450 \\
104807.210 \\
104993.860 \\
105123.000 \\
105220.600 \\
105524.460 \\
106018.640 \\
106097.520 \\
106722.170 \\
106900.370 \\
106924.430 \\
109625.200\end{array}$ & $\begin{array}{l}\left({ }^{3} \mathrm{H}\right) 4 \mathrm{~d}^{4} \mathrm{H}_{11 / 2} \\
\left({ }^{3} \mathrm{H}\right) 4 \mathrm{~d}^{4} \mathrm{G}_{9 / 2} \\
\left({ }^{3} \mathrm{H}\right) 4 \mathrm{~d}^{4} \mathrm{H}_{9 / 2} \\
\left({ }^{3} \mathrm{H}\right) 4 \mathrm{~d}^{4} \mathrm{G}_{7 / 2} \\
\left({ }^{3} \mathrm{H}\right) 4 \mathrm{~d}^{4} \mathrm{~K}_{11 / 2} \\
\left({ }^{3} \mathrm{G}\right) 5 \mathrm{~s}^{2} \mathrm{G}_{7 / 2} \\
\left({ }^{3} \mathrm{H}\right) 4 \mathrm{~d}^{4} \mathrm{H}_{7 / 2} \\
\left({ }^{3} \mathrm{P}\right) 4 \mathrm{~d}^{4} \mathrm{~F}_{7 / 2} \\
\left({ }^{3} \mathrm{H}\right) 4 \mathrm{~d}^{4} \mathrm{I}_{11 / 2} \\
\left.{ }^{3} \mathrm{H}\right) 4 \mathrm{~d}^{2} \mathrm{I}_{11 / 2} \\
\left({ }^{3} \mathrm{H}\right) 4 \mathrm{~d}^{2} \mathrm{G}_{9 / 2} \\
\left({ }^{3} \mathrm{~F}\right) 4 \mathrm{~d}^{4} \mathrm{D}_{7 / 2} \\
\left({ }^{3} \mathrm{H}\right) 4 \mathrm{~d}^{2} \mathrm{G}_{7 / 2} \\
\left({ }^{3} \mathrm{H}\right) 4 \mathrm{~d}^{4} \mathrm{~F}_{7 / 2} \\
\left({ }^{3} \mathrm{~F}\right) 4 \mathrm{~d}^{4} \mathrm{H}_{9 / 2} \\
\left({ }^{3} \mathrm{~F}\right) 4 \mathrm{~d}^{2} \mathrm{H}_{9 / 2} \\
\left({ }^{3} \mathrm{H}\right) 4 \mathrm{~d}^{2} \mathrm{H}_{9 / 2} \\
\left({ }^{3} \mathrm{~F}\right) 4 \mathrm{~d}^{4} \mathrm{~F}_{9 / 2} \\
\left({ }^{3} \mathrm{~F}\right) 4 \mathrm{~d}^{2} \mathrm{G}_{7 / 2} \\
\left({ }^{3} \mathrm{~F}\right) 4 \mathrm{~d}^{2} \mathrm{G}_{9 / 2} \\
\left({ }^{3} \mathrm{G}\right) 4 \mathrm{~d}^{2} \mathrm{G}_{9 / 2}\end{array}$ & $\begin{array}{l}5122.123 \\
5127.287 \\
5154.501 \\
5167.121 \\
5181.086 \\
5183.700 \\
5184.458 \\
5217.365 \\
5235.486 \\
5402.756 \\
5414.977 \\
5470.281 \\
5509.211 \\
5539.003 \\
5633.853 \\
5795.246 \\
5821.868 \\
6041.643 \\
6107.415 \\
6116.405 \\
7327.115\end{array}$ & $\begin{array}{l}-1.173 \\
-0.734 \\
+0.418 \\
-0.470 \\
-0.545 \\
-0.079 \\
-0.485 \\
-1.017 \\
-0.560 \\
-0.812 \\
-0.955 \\
-1.409 \\
-0.290 \\
-1.382 \\
-1.381 \\
-0.974 \\
-0.325 \\
-1.431 \\
-0.980 \\
-0.472 \\
-1.238\end{array}$ & $\begin{array}{l}5154.50 \\
5167.1 \\
5181.1 \\
5184.463\end{array}$ & $\begin{array}{l}\text { blend } \\
\text { blend } \\
\text { lab } \\
\text { computed too weak } \\
\text { blend, computed too weak } \\
\text { blend } \\
\text { computed too strong }\end{array}$ \\
\hline 123258.994 & $\left({ }^{3} \mathrm{H}\right) 4 \mathrm{f}$ & $5[4]$ & $9 / 2$ & $\begin{array}{l}103165.320 \\
103191.917 \\
103600.430 \\
103683.070 \\
103751.660\end{array}$ & $\begin{array}{l}\left({ }^{3} \mathrm{P}\right) 4 \mathrm{~d}^{4} \mathrm{~F}_{9 / 2} \\
\left({ }^{3} \mathrm{P}\right) 4 \mathrm{~d}^{2} \mathrm{~F}_{7 / 2} \\
\left({ }^{3} \mathrm{H}\right) 4 \mathrm{~d}^{4} \mathrm{G}_{11 / 2} \\
\left({ }^{3} \mathrm{H}\right) 4 \mathrm{~d}^{4} \mathrm{~F}_{9 / 2} \\
\left({ }^{3} \mathrm{H}\right) 4 \mathrm{~d}^{4} \mathrm{H}_{11 / 2}\end{array}$ & $\begin{array}{l}4975.303 \\
4981.898 \\
5085.425 \\
5106.894 \\
5124.850\end{array}$ & $\begin{array}{l}-1.479 \\
-0.587 \\
-1.404 \\
-0.960 \\
+0.047\end{array}$ & 5124.82 & lab \\
\hline
\end{tabular}


Table 7. continued.

\begin{tabular}{|c|c|c|c|c|c|c|c|c|c|}
\hline \multicolumn{4}{|c|}{ Upper level } & \multicolumn{2}{|c|}{ Lower level } & \multirow{2}{*}{$\begin{array}{c}\lambda(\text { calc }) \\
\AA\end{array}$} & \multirow{2}{*}{$\begin{array}{c}\log g f \\
\text { KUR }\end{array}$} & \multirow{2}{*}{$\begin{array}{c}\lambda(\mathrm{obs}) \\
\AA \\
\end{array}$} & \multirow[t]{2}{*}{ Notes } \\
\hline $\mathrm{cm}^{-1}$ & & & $J$ & $\mathrm{~cm}^{-1}$ & & & & & \\
\hline 123258.994 & cont. & & & $\begin{array}{l}103771.320 \\
103874.260 \\
104481.590 \\
104807.210 \\
104916.550 \\
105063.550 \\
105123.000 \\
105155.090 \\
105211.062 \\
105763.270 \\
106045.690 \\
106097.520 \\
106722.170 \\
106767.210 \\
106900.370 \\
108391.500 \\
109683.280 \\
110167.280\end{array}$ & $\begin{array}{l}\left({ }^{3} \mathrm{H}\right) 4 d^{4} \mathrm{G}_{9 / 2} \\
\left({ }^{3} \mathrm{H}\right) 4 \mathrm{~d}^{4} \mathrm{H}_{9 / 2} \\
\left({ }^{3} \mathrm{H}\right) 4 \mathrm{~d}^{2} \mathrm{~F}_{7 / 2} \\
\left({ }^{3} \mathrm{H}\right) 4 \mathrm{~d}^{2} \mathrm{G}_{9 / 2} \\
\left({ }^{3} \mathrm{H}\right) 4 \mathrm{~d}^{4} \mathrm{~F}_{9 / 2} \\
\left({ }^{3} \mathrm{~F}\right) 4 \mathrm{~d}^{4} \mathrm{G}_{11 / 2} \\
\left({ }^{3} \mathrm{H}\right) 4 \mathrm{~d}^{2} \mathrm{G}_{7 / 2} \\
\left({ }^{3} \mathrm{~F}\right) 4 \mathrm{~d}^{4} \mathrm{G}_{9 / 2} \\
\left({ }^{5} \mathrm{D}\right) 5 \mathrm{~d}^{4} \mathrm{G}_{9 / 2} \\
\left({ }^{3} \mathrm{~F}\right) 4 \mathrm{~d}^{2} \mathrm{H}_{11 / 2} \\
\left({ }^{3} \mathrm{H}\right) 4 \mathrm{~d}^{2} \mathrm{H}_{11 / 2} \\
\left({ }^{3} \mathrm{H}\right) 4 \mathrm{~d}^{2} \mathrm{H}_{9 / 2} \\
\left({ }^{3} \mathrm{~F}\right) 4 \mathrm{~d}^{4} \mathrm{~F}_{9 / 2} \\
\left({ }^{3} \mathrm{~F}\right) 4 \mathrm{~d}^{4} \mathrm{~F}_{7 / 2} \\
\left({ }^{3} \mathrm{~F}\right) 4 \mathrm{~d}^{2} \mathrm{G}_{7 / 2} \\
\left({ }^{3} \mathrm{G}\right) 4 \mathrm{~d}^{4} \mathrm{G}_{9 / 2} \\
\left({ }^{3} \mathrm{G}\right) 4 \mathrm{~d}^{2} \mathrm{H}_{11 / 2} \\
\left({ }^{3} \mathrm{G}\right) 4 \mathrm{~d}^{4} \mathrm{~F}_{7 / 2}\end{array}$ & $\begin{array}{l}5130.020 \\
5157.263 \\
5324.070 \\
5418.025 \\
5450.323 \\
5494.356 \\
5512.367 \\
5522.138 \\
5539.264 \\
5714.098 \\
5807.851 \\
5825.392 \\
6045.483 \\
6061.948 \\
6111.293 \\
6724.229 \\
7364.069 \\
7636.319\end{array}$ & $\begin{array}{l}+0.269 \\
-0.663 \\
-0.506 \\
-0.657 \\
+0.051 \\
-1.301 \\
-0.848 \\
-0.450 \\
-1.434 \\
-0.740 \\
-0.440 \\
-0.814 \\
-0.970 \\
-1.148 \\
-1.488 \\
-1.436 \\
-1.370 \\
-1.343\end{array}$ & $\begin{array}{l}5130.0 \\
5418.02 \\
5450.30 \\
5522.10 \\
\\
5714.10 \\
5807.85\end{array}$ & $\begin{array}{l}\text { lab } \\
\text { blend } \\
\text { blend } \\
\text { lab } \\
\text { wrong, computed too strong } \\
\text { computed too strong } \\
\text { blend }\end{array}$ \\
\hline 123258.021 & $\left({ }^{3} \mathrm{H}\right) 4 \mathrm{f}$ & $5[4]$ & $7 / 2$ & $\begin{array}{l}102802.312 \\
103002.670 \\
103102.860 \\
103771.320 \\
103874.260 \\
103921.630 \\
103983.510 \\
103986.330 \\
104107.950 \\
104120.270 \\
104209.610 \\
104569.230 \\
104916.550 \\
104993.860 \\
105123.000 \\
105220.600 \\
106018.640 \\
106097.520 \\
106866.760 \\
106900.370 \\
106924.430 \\
110167.280\end{array}$ & $\begin{array}{l}\left({ }^{5} \mathrm{D}\right) 6 \mathrm{~s}{ }^{4} \mathrm{D}_{5 / 2} \\
\left({ }^{3} \mathrm{P}\right) 4 \mathrm{~d}^{4} \mathrm{D}_{5 / 2} \\
\left({ }^{3} \mathrm{P}\right) 4 \mathrm{~d}^{4} \mathrm{D}_{7 / 2} \\
\left({ }^{3} \mathrm{H}\right) 4 \mathrm{~d}^{4} \mathrm{G}_{9 / 2} \\
\left({ }^{3} \mathrm{H}\right) 4 \mathrm{~d}^{4} \mathrm{H}_{9 / 2} \\
\left({ }^{3} \mathrm{H}\right) 4 \mathrm{~d}^{4} \mathrm{G}_{7 / 2} \\
\left({ }^{3} \mathrm{G}\right) 5 \mathrm{~s}^{2} \mathrm{G}_{7 / 2} \\
\left({ }^{3} \mathrm{H}\right) 4 \mathrm{~d}^{4} \mathrm{H}_{7 / 2} \\
\left({ }^{3} \mathrm{P}\right) 4 \mathrm{~d}^{4} \mathrm{~F}_{7 / 2} \\
\left({ }^{5} \mathrm{D}\right) 5 \mathrm{~d}^{6} \mathrm{P}_{5 / 2} \\
\left({ }^{3} \mathrm{H}\right) 4 \mathrm{~d}^{2} \mathrm{~F}_{5 / 2} \\
\left({ }^{3} \mathrm{P}\right) 4 \mathrm{~d}^{4} \mathrm{~F}_{5 / 2} \\
\left({ }^{3} \mathrm{H}\right) 4 \mathrm{~d}^{4} \mathrm{~F}_{9 / 2} \\
\left({ }^{3} \mathrm{~F}\right) 4 \mathrm{~d}^{4} \mathrm{D}_{7 / 2} \\
\left({ }^{3} \mathrm{H}\right) 4 \mathrm{~d}^{2} \mathrm{G}_{7 / 2} \\
\left({ }^{3} \mathrm{H}\right) 4 \mathrm{~d}^{4} \mathrm{~F}_{7 / 2} \\
\left({ }^{3} \mathrm{~F}\right) 4 \mathrm{~d}^{2} \mathrm{H}_{9 / 2} \\
\left({ }^{3} \mathrm{H}\right) 4 \mathrm{~d}^{2} \mathrm{H}_{9 / 2} \\
\left({ }^{3} \mathrm{~F}\right) 4 \mathrm{~d}^{4} \mathrm{~F}_{5 / 2} \\
\left({ }^{3} \mathrm{~F}\right) 4 \mathrm{~d}^{2} \mathrm{G}_{7 / 2} \\
\left({ }^{3} \mathrm{~F}\right) 4 \mathrm{~d}^{2} \mathrm{G}_{9 / 2} \\
\left({ }^{3} \mathrm{G}\right) 4 \mathrm{~d}^{4} \mathrm{~F}_{7 / 2}\end{array}$ & $\begin{array}{l}4887.246 \\
4935.589 \\
4960.124 \\
5130.276 \\
5157.521 \\
5170.156 \\
5186.755 \\
5187.514 \\
5220.459 \\
5223.820 \\
5248.321 \\
5349.313 \\
5450.611 \\
5473.683 \\
5512.661 \\
5542.490 \\
5799.064 \\
5825.721 \\
6099.124 \\
6111.655 \\
6120.658 \\
7636.885\end{array}$ & $\begin{array}{l}-1.497 \\
-1.223 \\
-1.397 \\
-0.633 \\
-0.254 \\
-0.375 \\
-0.078 \\
-0.396 \\
-1.202 \\
-0.829 \\
-0.898 \\
-0.940 \\
-1.412 \\
-0.926 \\
+0.003 \\
-1.205 \\
-1.320 \\
-0.559 \\
-1.189 \\
-0.698 \\
-0.942 \\
-1.434\end{array}$ & $\begin{array}{l}5512.65 \\
5825.73\end{array}$ & $\begin{array}{l}\text { blend } \\
\text { blend } \\
\text { at the continuum level } \\
\text { blend } \\
\text { blend } \\
\text { blend } \\
\text { blend } \\
\text { computed too strong } \\
\text { blend } \\
\text { blend } \\
\text { wrong, not observed } \\
\text { blend } \\
\text { blend } \\
\text { blend } \\
\text { blend } \\
\text { blend } \\
\text { blend } \\
\text { at the continuum level } \\
\text { no spectrum }\end{array}$ \\
\hline 123235.165 & $\left({ }^{3} \mathrm{H}\right) 4 \mathrm{f}$ & $5[3]$ & $7 / 2$ & $\begin{array}{l}103191.917 \\
103771.320 \\
103874.260 \\
103921.630 \\
103983.510 \\
103986.330 \\
104107.950 \\
104481.590 \\
104807.210 \\
104916.550 \\
105123.000 \\
105155.090 \\
105220.600 \\
105291.010 \\
106722.170\end{array}$ & $\begin{array}{l}\left({ }^{3} \mathrm{P}\right) 4 \mathrm{~d}^{2} \mathrm{~F}_{7 / 2} \\
\left({ }^{3} \mathrm{H}\right) 4 d^{4} \mathrm{G}_{9 / 2} \\
\left({ }^{3} \mathrm{H}\right) 4 \mathrm{~d}^{4} \mathrm{H}_{9 / 2} \\
\left({ }^{3} \mathrm{H}\right) 4 \mathrm{~d}^{4} \mathrm{G}_{7 / 2} \\
\left({ }^{3} \mathrm{G}\right) 5 \mathrm{~s}^{2} \mathrm{G}_{7 / 2} \\
\left({ }^{3} \mathrm{H}\right) 4 \mathrm{~d}^{4} \mathrm{H}_{7 / 2} \\
\left({ }^{3} \mathrm{P}\right) 4 \mathrm{~d}^{4} \mathrm{~F}_{7 / 2} \\
\left({ }^{3} \mathrm{H}\right) 4 \mathrm{~d}^{2} \mathrm{~F}_{7 / 2} \\
\left({ }^{3} \mathrm{H}\right) 4 \mathrm{~d}^{2} \mathrm{G}_{9 / 2} \\
\left({ }^{3} \mathrm{H}\right) 4 \mathrm{~d}^{4} \mathrm{~F}_{9 / 2} \\
\left({ }^{3} \mathrm{H}\right) 4 \mathrm{~d}^{2} \mathrm{G}_{7 / 2} \\
\left({ }^{3} \mathrm{~F}\right) 4 \mathrm{~d}^{4} \mathrm{G}_{9 / 2} \\
\left({ }^{3} \mathrm{H}\right) 4 \mathrm{~d}^{4} \mathrm{~F}_{7 / 2} \\
\left({ }^{3} \mathrm{~F}\right) 4 \mathrm{~d}^{4} \mathrm{G}_{7 / 2} \\
\left({ }^{3} \mathrm{~F}\right) 4 \mathrm{~d}^{4} \mathrm{~F}_{9 / 2}\end{array}$ & $\begin{array}{l}4987.820 \\
5136.300 \\
5163.610 \\
5176.274 \\
5192.913 \\
5193.673 \\
5226.698 \\
5330.834 \\
5425.030 \\
5457.411 \\
5519.618 \\
5529.415 \\
5549.523 \\
5571.298 \\
6054.160\end{array}$ & $\begin{array}{l}-0.173 \\
-0.037 \\
-0.154 \\
-0.716 \\
-0.799 \\
-0.887 \\
-1.309 \\
-0.226 \\
-0.825 \\
-0.238 \\
-1.438 \\
-0.668 \\
-1.242 \\
-1.482 \\
-1.224\end{array}$ & $\begin{array}{l}5330.81 \\
5425.01 \\
5457.40 \\
5529.40\end{array}$ & $\begin{array}{l}\text { blend } \\
\text { blend } \\
\text { blend } \\
\text { computed too strong }\end{array}$ \\
\hline
\end{tabular}


F. Castelli and R. L. Kurucz: New Fe II energy levels from stellar spectra

Table 7. continued.

\begin{tabular}{|c|c|c|c|c|c|c|c|c|c|}
\hline \multicolumn{4}{|c|}{ Upper level } & \multicolumn{2}{|c|}{ Lower level } & \multirow{2}{*}{$\begin{array}{c}\lambda(\text { calc }) \\
\AA\end{array}$} & \multirow{2}{*}{$\begin{array}{c}\log g f \\
\text { KUR }\end{array}$} & \multirow{2}{*}{$\begin{array}{c}\lambda(\mathrm{obs}) \\
\AA\end{array}$} & \multirow[t]{2}{*}{ Notes } \\
\hline $\mathrm{cm}^{-1}$ & & & $J$ & $\mathrm{~cm}^{-1}$ & & & & & \\
\hline 123235.165 & cont. & & & $\begin{array}{l}106767.210 \\
110167.280 \\
110570.300\end{array}$ & $\begin{array}{l}\left({ }^{3} \mathrm{~F}\right) 4 d^{4} F_{7 / 2} \\
\left({ }^{3} \mathrm{G}\right) 4 d^{4} F_{7 / 2} \\
\left({ }^{3} G\right) 4 d^{2} F_{7 / 2}\end{array}$ & $\begin{array}{l}6070.719 \\
7650.242 \\
7893.688\end{array}$ & $\begin{array}{l}-0.626 \\
-0.970 \\
-1.448\end{array}$ & 6070.71 & \\
\hline 123211.159 & $\left({ }^{3} \mathrm{H}\right) 4 \mathrm{f}$ & $5[2]$ & $5 / 2$ & $\begin{array}{l}103193.917 \\
103921.630 \\
103986.330 \\
104481.590 \\
104993.860 \\
105123.000 \\
105291.010 \\
106767.210 \\
106900.370 \\
110167.280 \\
110570.300\end{array}$ & $\begin{array}{l}\left({ }^{3} \mathrm{P}\right) 4 d^{2} \mathrm{~F}_{7 / 2} \\
\left({ }^{3} \mathrm{H}\right) 4 \mathrm{~d}^{4} \mathrm{G}_{7 / 2} \\
\left({ }^{3} \mathrm{G}\right) 5 \mathrm{~s}^{2} \mathrm{G}_{7 / 2} \\
\left({ }^{3} \mathrm{H}\right) 4 \mathrm{~d}^{2} \mathrm{~F}_{7 / 2} \\
\left({ }^{3} \mathrm{~F}\right) 4 \mathrm{~d}^{4} \mathrm{D}_{7 / 2} \\
\left({ }^{3} \mathrm{H}\right) 4 \mathrm{~d}^{2} \mathrm{G}_{7 / 2} \\
\left({ }^{3} \mathrm{~F}\right) 4 \mathrm{~d}^{4} \mathrm{G}_{7 / 2} \\
\left({ }^{3} \mathrm{~F}\right) 4 \mathrm{~d}^{4} \mathrm{~F}_{7 / 2} \\
\left({ }^{3} \mathrm{~F}\right) 4 \mathrm{~d}^{2} \mathrm{G}_{7 / 2} \\
\left({ }^{3} \mathrm{G}\right) 4 d^{4} \mathrm{~F}_{7 / 2} \\
\left({ }^{3} \mathrm{G}\right) 4 d^{2} \mathrm{~F}_{7 / 2}\end{array}$ & $\begin{array}{l}4993.801 \\
5182.716 \\
5200.159 \\
5337.666 \\
5487.763 \\
5526.943 \\
5578.762 \\
6079.581 \\
6129.215 \\
7664.321 \\
7908.679\end{array}$ & $\begin{array}{l}-0.145 \\
-1.163 \\
-1.442 \\
-0.236 \\
-1.396 \\
-0.560 \\
-1.365 \\
-0.532 \\
-1.126 \\
-0.703 \\
-1.384\end{array}$ & $\begin{array}{l}5526.92 \\
6709.60\end{array}$ & $\begin{array}{l}\text { computed too strong } \\
\text { good agreement } \\
\text { blend } \\
\text { blend } \\
\text { computed too strong } \\
\text { at the level of the noise } \\
\text { good agreement } \\
\text { blend } \\
\text { in telluric } \\
\text { in telluric }\end{array}$ \\
\hline 123213.323 & $\left({ }^{3} \mathrm{H}\right) 4 \mathrm{f}$ & $5[2]$ & $3 / 2$ & $\begin{array}{l}102802.312 \\
103597.402 \\
104120.270 \\
104209.610 \\
104569.230 \\
105234.237 \\
105414.180 \\
106796.660 \\
106866.760 \\
110428.280\end{array}$ & $\begin{array}{l}\left({ }^{5} \mathrm{D}\right) 6 \mathrm{~s}^{4} \mathrm{D}_{5 / 2} \\
\left({ }^{3} \mathrm{P}\right) 4 \mathrm{~d}^{2} \mathrm{D}_{5 / 2} \\
\left({ }^{5} \mathrm{D}\right) 5 \mathrm{~d}^{6} \mathrm{P}_{5 / 2} \\
\left({ }^{3} \mathrm{H}\right) 4 \mathrm{~d}^{2} \mathrm{~F}_{5 / 2} \\
\left({ }^{3} \mathrm{P}\right) 4 \mathrm{~d}^{4} \mathrm{~F}_{5 / 2} \\
\left({ }^{3} \mathrm{H}\right) 4 \mathrm{~d}^{4} \mathrm{~F}_{5 / 2} \\
\left({ }^{3} \mathrm{~F}\right) 4 \mathrm{~d}^{4} \mathrm{G}_{5 / 2} \\
\left({ }^{3} \mathrm{~F}\right) 4 \mathrm{~d}^{4} \mathrm{P}_{5 / 2} \\
\left({ }^{3} \mathrm{~F}\right) 4 \mathrm{~d}^{4} \mathrm{~F}_{5 / 2} \\
\left({ }^{3} \mathrm{G}\right) 4 \mathrm{~d}^{4} \mathrm{~F}_{5 / 2}\end{array}$ & $\begin{array}{l}4897.949 \\
5096.480 \\
5236.050 \\
5260.666 \\
5362.139 \\
5560.475 \\
5616.690 \\
6089.687 \\
6115.802 \\
7819.490\end{array}$ & $\begin{array}{l}-1.090 \\
-1.325 \\
-0.269 \\
-0.338 \\
-0.684 \\
-1.142 \\
-1.055 \\
-1.322 \\
-0.758 \\
-1.269\end{array}$ & $\begin{array}{l}4897.90 \\
5236.046 \\
5260.682\end{array}$ & $\begin{array}{l}\text { at the level of the noise } \\
\text { at the level of the noise } \\
\text { computed too strong } \\
\text { lab, good agreement } \\
\text { wrong, not observed } \\
\text { blend } \\
\text { blend } \\
\text { good agreement } \\
\text { at the continuum level }\end{array}$ \\
\hline 123396.250 & $\left({ }^{3} \mathrm{H}\right) 4 \mathrm{f}$ & $4[7]$ & $15 / 2$ & $\begin{array}{l}103706.530 \\
103832.050 \\
104064.670 \\
104315.370 \\
104622.300\end{array}$ & $\begin{array}{l}\left({ }^{3} \mathrm{H}\right) 4 \mathrm{~d}^{4} \mathrm{~K}_{15 / 2} \\
\left({ }^{3} \mathrm{H}\right) 4 \mathrm{~d}^{4} \mathrm{~K}_{13 / 2} \\
\left({ }^{3} \mathrm{H}\right) 4 \mathrm{~d}^{4} \mathrm{I}_{13 / 2} \\
\left({ }^{3} \mathrm{H}\right) 4 \mathrm{~d}^{2} \mathrm{~K}_{13 / 2} \\
\left({ }^{3} \mathrm{H}\right) 4 \mathrm{~d}^{2} \mathrm{I}_{13 / 2}\end{array}$ & $\begin{array}{l}5077.377 \\
5109.953 \\
5171.443 \\
5239.390 \\
5325.048\end{array}$ & $\begin{array}{l}-1.404 \\
-0.102 \\
+0.259 \\
+0.861 \\
+0.257\end{array}$ & $\begin{array}{l}5109.95 \\
5171.45 \\
5239.394 \\
5325.05\end{array}$ & $\begin{array}{l}\text { lab } \\
\text { lab } \\
\text { J78 } \\
\text { J78, lab }\end{array}$ \\
\hline 123355.490 & $\left({ }^{3} \mathrm{H}\right) 4 \mathrm{f}$ & $4[7]$ & $13 / 2$ & $\begin{array}{l}103600.430 \\
103751.660 \\
103832.050 \\
103973.780 \\
104064.670 \\
104119.710 \\
104315.370 \\
104622.300 \\
104765.450 \\
105763.270 \\
106045.690 \\
109683.280\end{array}$ & $\begin{array}{l}\left({ }^{3} \mathrm{H}\right) 4 d^{4} \mathrm{G}_{11 / 2} \\
\left({ }^{3} \mathrm{H}\right) 4 \mathrm{~d}^{4} \mathrm{H}_{11 / 2} \\
\left({ }^{3} \mathrm{H}\right) 4 \mathrm{~d}^{4} \mathrm{~K}_{13 / 2} \\
\left({ }^{3} \mathrm{H}\right) 4 \mathrm{~d}^{4} \mathrm{~K}_{11 / 2} \\
\left({ }^{3} \mathrm{H}\right) 4 \mathrm{~d}^{4} \mathrm{I}_{13 / 2} \\
\left({ }^{3} \mathrm{H}\right) 4 \mathrm{~d}^{2} \mathrm{~K}_{15 / 2} \\
\left({ }^{3} \mathrm{H}\right) 4 \mathrm{~d}^{2} \mathrm{~K}_{13 / 2} \\
\left({ }^{3} \mathrm{H}\right) 4 \mathrm{~d}^{2} \mathrm{I}_{13 / 2} \\
\left({ }^{3} \mathrm{H}\right) 4 \mathrm{~d}^{2} \mathrm{I}_{11 / 2} \\
\left({ }^{3} \mathrm{~F}\right) 4 \mathrm{~d}^{2} \mathrm{H}_{11 / 2} \\
\left({ }^{3} \mathrm{H}\right) 4 \mathrm{~d}^{2} \mathrm{H}_{11 / 2} \\
\left({ }^{3} \mathrm{G}\right) 4 \mathrm{~d}^{2} \mathrm{H}_{11 / 2}\end{array}$ & $\begin{array}{l}5060.583 \\
5099.623 \\
5120.621 \\
5158.067 \\
5182.370 \\
5197.198 \\
5250.606 \\
5336.635 \\
5377.729 \\
5682.754 \\
5775.473 \\
7312.092\end{array}$ & $\begin{array}{l}-1.409 \\
-0.221 \\
-1.170 \\
+0.788 \\
+0.034 \\
-1.475 \\
-0.778 \\
-0.215 \\
-0.165 \\
-0.574 \\
-0.674 \\
-1.277\end{array}$ & $\begin{array}{l}5099.6 \\
5120.62 \\
5158.05 \\
5182.371 \\
\\
5250.609 \\
5336.62 \\
5377.71 \\
5682.75\end{array}$ & $\begin{array}{l}\text { lab } \\
\text { lab, computed too weak } \\
\mathrm{J} 78, \text { lab } \\
\text { lab } \\
\text { computed too weak } \\
\text { J78, lab, computed too weak }\end{array}$ \\
\hline 123414.730 & $\left({ }^{3} \mathrm{H}\right) 4 \mathrm{f}$ & $4[6]$ & $13 / 2$ & $\begin{array}{l}103751.660 \\
103832.050 \\
103973.780 \\
104064.670 \\
104174.270 \\
104315.370 \\
104622.300 \\
104765.450 \\
105063.550 \\
105398.850 \\
106045.690\end{array}$ & $\begin{array}{l}\left({ }^{3} \mathrm{H}\right) 4 \mathrm{~d}^{4} \mathrm{H}_{11 / 2} \\
\left({ }^{3} \mathrm{H}\right) 4 \mathrm{~d}^{4} \mathrm{~K}_{13 / 2} \\
\left({ }^{3} \mathrm{H}\right) 4 \mathrm{~d}^{4} \mathrm{~K}_{11 / 2} \\
\left({ }^{3} \mathrm{H}\right) 4 \mathrm{~d}^{4} \mathrm{I}_{13 / 2} \\
\left({ }^{3} \mathrm{H}\right) 4 \mathrm{~d}^{4} \mathrm{I}_{11 / 2} \\
\left({ }^{3} \mathrm{H}\right) 4 \mathrm{~d}^{2} \mathrm{~K}_{13 / 2} \\
\left({ }^{3} \mathrm{H}\right) 4 \mathrm{~d}^{2} \mathrm{I}_{13 / 2} \\
\left({ }^{3} \mathrm{H}\right) 4 \mathrm{~d}^{2} \mathrm{I}_{11 / 2} \\
\left({ }^{3} \mathrm{~F}\right) 4 \mathrm{~d}^{4} \mathrm{G}_{11 / 2} \\
\left({ }^{3} \mathrm{~F}\right) 4 \mathrm{~d}^{4} \mathrm{H}_{11 / 2} \\
\left({ }^{3} \mathrm{H}\right) 4 \mathrm{~d}^{2} \mathrm{H}_{11 / 2}\end{array}$ & $\begin{array}{l}5084.259 \\
5105.131 \\
5142.349 \\
5166.504 \\
5195.934 \\
5234.320 \\
5319.812 \\
5360.646 \\
5447.727 \\
5549.118 \\
5755.774\end{array}$ & $\begin{array}{l}-0.750 \\
-0.704 \\
-0.245 \\
-0.525 \\
+0.922 \\
-0.791 \\
-1.134 \\
-0.638 \\
-1.416 \\
-1.185 \\
-1.242\end{array}$ & $\begin{array}{l}5142.35 \\
5195.942\end{array}$ & $\begin{array}{l}\text { lab } \\
\text { blend } \\
\text { lab } \\
\text { blend } \\
\text { computed too weak }\end{array}$ \\
\hline
\end{tabular}


Table 7. continued.

\begin{tabular}{|c|c|c|c|c|c|c|c|c|c|}
\hline \multicolumn{4}{|c|}{ Upper level } & \multicolumn{2}{|c|}{ Lower level } & \multirow{2}{*}{$\begin{array}{c}\lambda(\text { calc }) \\
\AA \\
\end{array}$} & \multirow{2}{*}{$\begin{array}{c}\log g f \\
\text { KUR }\end{array}$} & \multirow{2}{*}{$\begin{array}{c}\lambda \text { (obs) } \\
\AA \\
\end{array}$} & \multirow[t]{2}{*}{ Notes } \\
\hline $\mathrm{cm}^{-1}$ & & & $J$ & $\mathrm{~cm}^{-1}$ & & & & & \\
\hline 123427.119 & $\left({ }^{3} \mathrm{H}\right) 4 \mathrm{f}$ & $4[6]$ & $11 / 2$ & $\begin{array}{l}103771.320 \\
103874.260 \\
103973.780 \\
104192.480 \\
104315.370 \\
104622.300 \\
104765.450 \\
104807.210 \\
105063.550 \\
105763.270 \\
106018.640 \\
106045.690 \\
106097.520 \\
106722.170 \\
106924.430 \\
109625.200 \\
110008.300\end{array}$ & $\begin{array}{l}\left({ }^{3} \mathrm{H}\right) 4 d^{4} \mathrm{G}_{9 / 2} \\
\left({ }^{3} \mathrm{H}\right) 4 \mathrm{~d}^{4} \mathrm{H}_{9 / 2} \\
\left({ }^{3} \mathrm{H}\right) 4 \mathrm{~d}^{4} \mathrm{~K}_{11 / 2} \\
\left({ }^{3} \mathrm{H}\right) 4 \mathrm{~d}^{4} \mathrm{I}_{9 / 2} \\
\left({ }^{3} \mathrm{H}\right) 4 \mathrm{~d}^{2} \mathrm{~K}_{13 / 2} \\
\left({ }^{3} \mathrm{H}\right) 4 \mathrm{~d}^{2} \mathrm{I}_{13 / 2} \\
\left({ }^{3} \mathrm{H}\right) 4 \mathrm{~d}^{2} \mathrm{I}_{11 / 2} \\
\left({ }^{3} \mathrm{H}\right) 4 \mathrm{~d}^{2} \mathrm{G}_{9 / 2} \\
\left({ }^{3} \mathrm{~F}\right) 4 \mathrm{~d}^{4} \mathrm{G}_{11 / 2} \\
\left({ }^{3} \mathrm{~F}\right) 4 \mathrm{~d}^{2} \mathrm{H}_{11 / 2} \\
\left({ }^{3} \mathrm{~F}\right) 4 \mathrm{~d}^{2} \mathrm{H}_{9 / 2} \\
\left({ }^{3} \mathrm{H}\right) 4 \mathrm{~d}^{2} \mathrm{H}_{11 / 2} \\
\left({ }^{3} \mathrm{H}\right) 4 \mathrm{~d}^{2} \mathrm{H}_{9 / 2} \\
\left({ }^{3} \mathrm{~F}\right) 4 \mathrm{~d}^{4} \mathrm{~F}_{9 / 2} \\
\left({ }^{3} \mathrm{~F}\right) 4 \mathrm{~d}^{2} \mathrm{G}_{9 / 2} \\
\left({ }^{3} \mathrm{G}\right) 4 \mathrm{~d}^{2} \mathrm{G}_{9 / 2} \\
\left({ }^{3} \mathrm{G}\right) 4 \mathrm{~d}^{2} \mathrm{H}_{9 / 2}\end{array}$ & $\begin{array}{l}5086.139 \\
5112.917 \\
5139.074 \\
5197.506 \\
5230.927 \\
5316.307 \\
5357.088 \\
5369.102 \\
5444.051 \\
5659.712 \\
5742.735 \\
5751.672 \\
5768.874 \\
5984.595 \\
6057.941 \\
7243.378 \\
7450.174\end{array}$ & $\begin{array}{l}-0.441 \\
-0.423 \\
+0.124 \\
+0.465 \\
-1.051 \\
-1.253 \\
+0.165 \\
-1.260 \\
-0.902 \\
-0.911 \\
-0.704 \\
-1.454 \\
-0.115 \\
-1.089 \\
-0.358 \\
-1.142 \\
-1.329\end{array}$ & $\begin{array}{l}5086.15 \\
5139.10 \\
5197.56\end{array}$ & $\begin{array}{l}\text { J78,lab } \\
\text { computed too strong } \\
\text { J78, computed too weak } \\
\text { blend }\end{array}$ \\
\hline 123441.100 & $\left({ }^{3} \mathrm{H}\right) 4 \mathrm{f}$ & $4[5]$ & $11 / 2$ & $\begin{array}{l}103771.320 \\
103874.260 \\
103973.780 \\
104174.270 \\
104192.480 \\
104315.370 \\
104765.450 \\
105063.550 \\
105524.460 \\
106018.640 \\
106097.520 \\
106722.170 \\
106924.430 \\
109625.200\end{array}$ & $\begin{array}{l}\left({ }^{3} \mathrm{H}\right) 4 \mathrm{~d}^{4} \mathrm{G}_{9 / 2} \\
\left({ }^{3} \mathrm{H}\right) 4 \mathrm{~d}^{4} \mathrm{H}_{9 / 2} \\
\left({ }^{3} \mathrm{H}\right) 4 \mathrm{~d}^{4} \mathrm{~K}_{11 / 2} \\
\left({ }^{3} \mathrm{H}\right) 4 \mathrm{~d}^{4} \mathrm{I}_{11 / 2} \\
\left({ }^{3} \mathrm{H}\right) 4 \mathrm{~d}^{4} \mathrm{I}_{9 / 2} \\
\left({ }^{3} \mathrm{H}\right) 4 \mathrm{~d}^{2} \mathrm{~K}_{13 / 2} \\
\left({ }^{3} \mathrm{H}\right) 4 \mathrm{~d}^{2} \mathrm{I}_{11 / 2} \\
\left({ }^{3} \mathrm{~F}\right) 4 \mathrm{~d}^{4} \mathrm{G}_{11 / 2} \\
\left({ }^{3} \mathrm{~F}\right) 4 \mathrm{~d}^{4} \mathrm{H}_{9 / 2} \\
\left({ }^{3} \mathrm{~F}\right) 4 \mathrm{~d}^{2} \mathrm{H}_{9 / 2} \\
\left({ }^{3} \mathrm{H}\right) 4 \mathrm{~d}^{2} \mathrm{H}_{9 / 2} \\
\left({ }^{3} \mathrm{~F}\right) 4 \mathrm{~d}^{4} \mathrm{~F}_{9 / 2} \\
\left({ }^{3} \mathrm{~F}\right) 4 \mathrm{~d}^{2} \mathrm{G}_{9 / 2} \\
\left({ }^{3} \mathrm{G}\right) 4 \mathrm{~d}^{2} \mathrm{G}_{9 / 2}\end{array}$ & $\begin{array}{l}5082.524 \\
5109.263 \\
5135.383 \\
5188.822 \\
5193.731 \\
5227.103 \\
5353.077 \\
5439.910 \\
5579.854 \\
5738.126 \\
5764.224 \\
5979.588 \\
6052.813 \\
7236.043\end{array}$ & $\begin{array}{l}-0.439 \\
+0.037 \\
-1.089 \\
+0.224 \\
+0.573 \\
-1.390 \\
-0.299 \\
-1.230 \\
-1.306 \\
-1.011 \\
-0.455 \\
-1.109 \\
-0.460 \\
-1.361\end{array}$ & $\begin{array}{l}5082.51 \\
5109.29 \\
5188.831 \\
5193.74\end{array}$ & $\begin{array}{l}\text { computed too strong } \\
\text { lab } \\
\text { lab } \\
\mathrm{J} 78 \text {, lab } \\
\text { blend } \\
\text { computed too strong, not obs }\end{array}$ \\
\hline 123435.468 & $\left({ }^{3} \mathrm{H}\right) 4 \mathrm{f}$ & $4[5]$ & $9 / 2$ & $\begin{array}{l}103921.630 \\
103973.780 \\
103983.510 \\
103986.330 \\
104107.950 \\
104174.270 \\
104192.480 \\
105589.670\end{array}$ & $\begin{array}{l}\left({ }^{3} \mathrm{H}\right) 4 d^{4} \mathrm{G}_{7 / 2} \\
\left({ }^{3} \mathrm{H}\right) 4 \mathrm{~d}^{4} \mathrm{~K}_{11 / 2} \\
\left({ }^{3} \mathrm{G}\right) 5 \mathrm{~s}^{2} \mathrm{G}_{7 / 2} \\
\left({ }^{3} \mathrm{H}\right) 4 \mathrm{~d}^{4} \mathrm{H}_{7 / 2} \\
\left({ }^{3} \mathrm{P}\right) 4 \mathrm{~d}^{4} \mathrm{~F}_{7 / 2} \\
\left({ }^{3} \mathrm{H}\right) 4 \mathrm{~d}^{4} \mathrm{I}_{11 / 2} \\
\left({ }^{3} \mathrm{H}\right) 4 \mathrm{~d}^{4} \mathrm{I}_{9 / 2} \\
\left({ }^{3} \mathrm{~F}\right) 4 \mathrm{~d}^{4} \mathrm{H}_{7 / 2}\end{array}$ & $\begin{array}{l}5123.141 \\
5136.869 \\
5139.439 \\
5140.184 \\
5172.529 \\
5190.340 \\
5195.251 \\
5602.005\end{array}$ & $\begin{array}{l}+0.119 \\
-0.836 \\
+0.314 \\
-0.208 \\
-1.242 \\
-1.319 \\
+0.450 \\
-1.242\end{array}$ & $\begin{array}{l}5123.190 \\
5140.2 \\
5195.26\end{array}$ & $\begin{array}{l}\text { blend } \\
\text { blend } \\
\text { blend } \\
\text { lab } \\
\text { lab }\end{array}$ \\
\hline 123460.690 & $\left({ }^{3} \mathrm{H}\right) 4 \mathrm{f}$ & $4[4]$ & $9 / 2$ & $\begin{array}{l}103191.917 \\
103771.320 \\
103874.260 \\
103921.630 \\
103973.780 \\
103983.510 \\
103986.330 \\
104174.27 \\
104481.590 \\
104765.450 \\
104807.210 \\
104993.860 \\
105063.550 \\
105123.000 \\
105220.600 \\
105291.010 \\
105449.540\end{array}$ & $\begin{array}{l}\left({ }^{3} \mathrm{P}\right) 4 \mathrm{~d}^{2} \mathrm{~F}_{7 / 2} \\
\left({ }^{3} \mathrm{H}\right) 4 \mathrm{~d}^{4} \mathrm{G}_{9 / 2} \\
\left({ }^{3} \mathrm{H}\right) 4 \mathrm{~d}^{4} \mathrm{H}_{9 / 2} \\
\left({ }^{3} \mathrm{H}\right) 4 \mathrm{~d}^{4} \mathrm{G}_{7 / 2} \\
\left({ }^{3} \mathrm{H}\right) 4 \mathrm{~d}^{4} \mathrm{~K}_{11 / 2} \\
\left({ }^{3} \mathrm{G}\right) 5 \mathrm{~s}^{2} \mathrm{G}_{7 / 2} \\
\left({ }^{3} \mathrm{H}\right) 4 \mathrm{~d}^{4} \mathrm{H}_{7 / 2} \\
\left({ }^{3} \mathrm{H}\right) 4 \mathrm{~d}^{4} \mathrm{I}_{11 / 2} \\
\left({ }^{3} \mathrm{H}\right) 4 \mathrm{~d}^{2} \mathrm{~F}_{7 / 2} \\
\left({ }^{3} \mathrm{H}\right) 4 \mathrm{~d}^{2} \mathrm{I}_{11 / 2} \\
\left({ }^{3} \mathrm{H}\right) 4 \mathrm{~d}^{2} \mathrm{G}_{9 / 2} \\
\left({ }^{3} \mathrm{~F}\right) 4 \mathrm{~d}^{4} \mathrm{D}_{7 / 2} \\
\left({ }^{3} \mathrm{~F}\right) 4 \mathrm{~d}^{4} \mathrm{G}_{11 / 2} \\
\left({ }^{3} \mathrm{H}\right) 4 \mathrm{~d}^{2} \mathrm{G}_{7 / 2} \\
\left({ }^{3} \mathrm{H}\right) 4 \mathrm{~d}^{4} \mathrm{~F}_{7 / 2} \\
\left({ }^{3} \mathrm{~F}\right) 4 \mathrm{~d}^{4} \mathrm{G}_{7 / 2} \\
\left({ }^{5} \mathrm{D}\right) 5 \mathrm{~d}^{4} \mathrm{G}_{7 / 2}\end{array}$ & $\begin{array}{l}4932.321 \\
5077.467 \\
5104.153 \\
5116.528 \\
5130.220 \\
5132.783 \\
5133.527 \\
5183.552 \\
5267.488 \\
5347.468 \\
5359.439 \\
5413.610 \\
5434.117 \\
5451.734 \\
5480.906 \\
5502.146 \\
5550.575\end{array}$ & $\begin{array}{l}-1.442 \\
-0.602 \\
-0.047 \\
-0.613 \\
-1.289 \\
-0.961 \\
-0.989 \\
-0.937 \\
-0.494 \\
-0.307 \\
-1.442 \\
-0.234 \\
-1.217 \\
-0.292 \\
-0.700 \\
-0.769 \\
-1.270\end{array}$ & $\begin{array}{l}5077.5 \\
5104.15 \\
5116.52 \\
\\
\\
5267.47 \\
5347.45 \\
5413.60 \\
5451.72\end{array}$ & $\begin{array}{l}\text { lab } \\
\text { lab }\end{array}$ \\
\hline
\end{tabular}


F. Castelli and R. L. Kurucz: New Fe II energy levels from stellar spectra

Table 7. continued.

\begin{tabular}{|c|c|c|c|c|c|c|c|c|c|}
\hline \multicolumn{4}{|c|}{ Upper level } & \multicolumn{2}{|c|}{ Lower level } & \multirow{2}{*}{$\begin{array}{c}\lambda(\mathrm{calc}) \\
\AA\end{array}$} & \multirow{2}{*}{$\begin{array}{c}\log g f \\
\text { KUR }\end{array}$} & \multirow{2}{*}{$\begin{array}{c}\lambda(\mathrm{obs}) \\
\AA\end{array}$} & \multirow[t]{2}{*}{ Notes } \\
\hline $\mathrm{cm}^{-1}$ & & & $J$ & $\mathrm{~cm}^{-1}$ & & & & & \\
\hline 123460.690 & cont. & & & $\begin{array}{l}106018.640 \\
106097.520 \\
106722.170 \\
106767.210 \\
106900.370 \\
106924.430 \\
109625.200 \\
110008.300\end{array}$ & $\begin{array}{l}\left({ }^{3} \mathrm{~F}\right) 4 \mathrm{~d}^{2} \mathrm{H}_{9 / 2} \\
\left({ }^{3} \mathrm{H}\right) 4 \mathrm{~d}^{2} \mathrm{H}_{9 / 2} \\
\left({ }^{3} \mathrm{~F}\right) 4 \mathrm{~d}^{4} \mathrm{~F}_{9 / 2} \\
\left({ }^{3} \mathrm{~F}\right) 4 \mathrm{~d}^{4} \mathrm{~F}_{7 / 2} \\
\left({ }^{3} \mathrm{~F}\right) 4 \mathrm{~d}^{2} \mathrm{G}_{7 / 2} \\
\left({ }^{3} \mathrm{~F}\right) 4 \mathrm{~d}^{2} \mathrm{G}_{9 / 2} \\
\left({ }^{3} \mathrm{G}\right) 4 \mathrm{~d}^{2} \mathrm{G}_{9 / 2} \\
\left({ }^{3} \mathrm{G}\right) 4 \mathrm{~d}^{2} \mathrm{H}_{9 / 2}\end{array}$ & $\begin{array}{l}5731.681 \\
5757.720 \\
5972.589 \\
5988.704 \\
6036.859 \\
6045.643 \\
7225.797 \\
7431.576\end{array}$ & $\begin{array}{l}-0.446 \\
+0.118 \\
-0.946 \\
-1.212 \\
-0.912 \\
-0.124 \\
-0.960 \\
-1.109\end{array}$ & 5757.72 & $\begin{array}{l}\text { wrong, not observed } \\
\mathrm{J} 78 \text {, computed too low }\end{array}$ \\
\hline 123435.277 & $\left({ }^{3} \mathrm{H}\right) 4 \mathrm{f}$ & $4[4]$ & $7 / 2$ & $\begin{array}{l}103921.630 \\
103983.510 \\
103986.330 \\
104023.910 \\
104120.270 \\
104192.480 \\
104209.610 \\
104569.230 \\
105414.180 \\
105589.670 \\
105630.750 \\
107407.800\end{array}$ & $\begin{array}{l}\left({ }^{3} \mathrm{H}\right) 4 d^{4} \mathrm{G}_{7 / 2} \\
\left({ }^{3} \mathrm{G}\right) 5 \mathrm{~s}^{2} \mathrm{G}_{7 / 2} \\
\left({ }^{3} \mathrm{H}\right) 4 \mathrm{~d}^{4} \mathrm{H}_{7 / 2} \\
\left({ }^{3} \mathrm{H}\right) 4 \mathrm{~d}^{4} \mathrm{G}_{5 / 2} \\
\left({ }^{5} \mathrm{D}\right) 5 \mathrm{~d}^{6} \mathrm{P}_{5 / 2} \\
\left({ }^{3} \mathrm{H}\right) 4 \mathrm{~d}^{4} \mathrm{I}_{9 / 2} \\
\left({ }^{3} \mathrm{H}\right) 4 \mathrm{~d}^{2} \mathrm{~F}_{5 / 2} \\
\left({ }^{3} \mathrm{P}\right) 4 \mathrm{~d}^{4} \mathrm{~F}_{5 / 2} \\
\left({ }^{3} \mathrm{~F}\right) 4 \mathrm{~d}^{4} \mathrm{G}_{5 / 2} \\
\left({ }^{3} \mathrm{~F}\right) 4 \mathrm{~d}^{4} \mathrm{H}_{7 / 2} \\
\left({ }^{5} \mathrm{D}\right) 5 \mathrm{~d}^{4} \mathrm{G}_{5 / 2} \\
\left({ }^{3} \mathrm{~F}\right) 4 \mathrm{~d}^{2} \mathrm{D}_{5 / 2}\end{array}$ & $\begin{array}{l}5123.191 \\
5139.489 \\
5140.234 \\
5150.186 \\
5175.880 \\
5195.303 \\
5199.932 \\
5299.053 \\
5547.511 \\
5602.065 \\
5614.990 \\
6237.560\end{array}$ & $\begin{array}{l}-0.068 \\
+0.217 \\
-0.435 \\
+0.144 \\
-1.206 \\
-0.325 \\
-1.066 \\
-0.753 \\
-1.009 \\
-1.328 \\
-1.423 \\
-1.471\end{array}$ & $\begin{array}{l}5139.45 \\
5140.20 \\
5150.15 \\
\\
5199.95\end{array}$ & $\begin{array}{l}\text { blend } \\
\text { lab, blend } \\
\text { blend } \\
\text { lab } \\
\text { blend } \\
\text { blend } \\
\text { computed too weak } \\
\text { computed too strong } \\
\text { at the level of the noise } \\
\text { blend } \\
\text { at the continuum level } \\
\text { at the continuum level }\end{array}$ \\
\hline 123451.449 & $\left({ }^{3} \mathrm{H}\right) 4 \mathrm{f}$ & $4[3]$ & $7 / 2$ & $\begin{array}{l}103191.917 \\
103597.402 \\
103771.320 \\
103874.260 \\
103921.630 \\
103983.510 \\
103986.330 \\
104023.910 \\
104107.950 \\
104120.270 \\
104481.590 \\
104569.230 \\
104993.860 \\
105123.000 \\
105220.600 \\
105291.010 \\
105449.540 \\
106018.640 \\
106097.520 \\
106767.210 \\
106900.370 \\
106924.430\end{array}$ & $\begin{array}{l}\left({ }^{3} \mathrm{P}\right) 4 \mathrm{~d}^{2} \mathrm{~F}_{7 / 2} \\
\left({ }^{3} \mathrm{P}\right) 4 \mathrm{~d}^{2} \mathrm{D}_{5 / 2} \\
\left({ }^{3} \mathrm{H}\right) 4 \mathrm{~d}^{4} \mathrm{G}_{9 / 2} \\
\left({ }^{3} \mathrm{H}\right) 4 \mathrm{~d}^{4} \mathrm{H}_{9 / 2} \\
\left({ }^{3} \mathrm{H}\right) 4 \mathrm{~d}^{4} \mathrm{G}_{7 / 2} \\
\left({ }^{3} \mathrm{G}\right) 5 \mathrm{~s}^{2} \mathrm{G}_{7 / 2} \\
\left({ }^{3} \mathrm{H}\right) 4 \mathrm{~d}^{4} \mathrm{H}_{7 / 2} \\
\left({ }^{3} \mathrm{H}\right) 4 \mathrm{~d}^{4} \mathrm{G}_{5 / 2} \\
\left({ }^{3} \mathrm{P}\right) 4 \mathrm{~d}^{4} \mathrm{~F}_{7 / 2} \\
\left({ }^{5} \mathrm{D}\right) 5 \mathrm{~d}^{6} \mathrm{P}_{5 / 2} \\
\left({ }^{3} \mathrm{H}\right) 4 \mathrm{~d}^{2} \mathrm{~F}_{7 / 2} \\
\left({ }^{3} \mathrm{P}\right) 4 \mathrm{~d}^{4} \mathrm{~F}_{5 / 2} \\
\left({ }^{3} \mathrm{~F}\right) 4 \mathrm{~d}^{4} \mathrm{D}_{7 / 2} \\
\left({ }^{3} \mathrm{H}\right) 4 \mathrm{~d}^{2} \mathrm{G}_{7 / 2} \\
\left({ }^{3} \mathrm{H}\right) 4 \mathrm{~d}^{4} \mathrm{~F}_{7 / 2} \\
\left({ }^{3} \mathrm{~F}\right) 4 \mathrm{~d}^{4} \mathrm{G}_{7 / 2} \\
\left({ }^{5} \mathrm{D}\right) 5 \mathrm{~d}^{4} \mathrm{G}_{7 / 2} \\
\left({ }^{3} \mathrm{~F}\right) 4 \mathrm{~d}^{2} \mathrm{H}_{9 / 2} \\
\left({ }^{3} \mathrm{H}\right) 4 \mathrm{~d}^{2} \mathrm{H}_{9 / 2} \\
\left({ }^{3} \mathrm{~F}\right) 4 \mathrm{~d}^{4} \mathrm{~F}_{7 / 2} \\
\left({ }^{3} \mathrm{~F}\right) 4 \mathrm{~d}^{2} \mathrm{G}_{7 / 2} \\
\left({ }^{3} \mathrm{~F}\right) 4 \mathrm{~d}^{2} \mathrm{G}_{9 / 2}\end{array}$ & $\begin{array}{l}4934.571 \\
5035.352 \\
5079.851 \\
5106.563 \\
5118.949 \\
5135.220 \\
5135.964 \\
5145.899 \\
5168.256 \\
5171.550 \\
5270.054 \\
5294.515 \\
5416.320 \\
5454.483 \\
5483.684 \\
5504.945 \\
5553.424 \\
5734.719 \\
5760.786 \\
5992.021 \\
6040.230 \\
6049.023\end{array}$ & $\begin{array}{l}-1.453 \\
-0.856 \\
-1.218 \\
-0.583 \\
-1.061 \\
-0.335 \\
-1.420 \\
-0.764 \\
-1.230 \\
-1.408 \\
-0.654 \\
-1.314 \\
-0.276 \\
-0.324 \\
-0.695 \\
-0.792 \\
-1.292 \\
-1.053 \\
-0.536 \\
-1.212 \\
-1.110 \\
-0.751\end{array}$ & $\begin{array}{l}5106.55 \\
5135.95\end{array}$ & $\begin{array}{l}\text { blend } \\
\text { lab } \\
\text { blend }\end{array}$ \\
\hline 123430.181 & $\left({ }^{3} \mathrm{H}\right) 4 \mathrm{f}$ & $4[3]$ & $5 / 2$ & $\begin{array}{l}103597.402 \\
103921.630 \\
103983.510 \\
103986.330 \\
104023.910 \\
104120.270 \\
104209.610 \\
104569.230 \\
104572.920 \\
104993.860 \\
105317.440 \\
\end{array}$ & $\begin{array}{l}\left({ }^{3} \mathrm{P}\right) 4 \mathrm{~d}^{2} \mathrm{D}_{5 / 2} \\
\left({ }^{3} \mathrm{H}\right) 4 \mathrm{~d}^{4} \mathrm{G}_{7 / 2} \\
\left({ }^{3} \mathrm{G}\right) 5 \mathrm{~s}^{2} \mathrm{G}_{7 / 2} \\
\left({ }^{3} \mathrm{H}\right) 4 \mathrm{~d}^{4} \mathrm{H}_{7 / 2} \\
\left({ }^{3} \mathrm{H}\right) 4 \mathrm{~d}^{4} \mathrm{G}_{5 / 2} \\
\left({ }^{5} \mathrm{D}\right) 5 \mathrm{~d}^{6} \mathrm{P}_{5 / 2} \\
\left({ }^{3} \mathrm{H}\right) 4 \mathrm{~d}^{2} \mathrm{~F}_{5 / 2} \\
\left({ }^{3} \mathrm{P}\right) 4 \mathrm{~d}^{4} \mathrm{~F}_{5 / 2} \\
\left({ }^{3} \mathrm{P}\right) 4 \mathrm{~d}^{4} \mathrm{~F}_{3 / 2} \\
\left({ }^{3} \mathrm{~F}\right) 4 \mathrm{~d}^{4} \mathrm{D}_{7 / 2} \\
\left({ }^{3} \mathrm{P}\right) 4 \mathrm{~d}^{2} \mathrm{P}_{3 / 2}\end{array}$ & $\begin{array}{l}5040.752 \\
5124.529 \\
5140.836 \\
5141.582 \\
5151.538 \\
5177.246 \\
5201.311 \\
5300.485 \\
5301.522 \\
5422.568 \\
5519.442 \\
\end{array}$ & $\begin{array}{l}-1.238 \\
-0.535 \\
-0.648 \\
-0.884 \\
+0.030 \\
-0.906 \\
-0.851 \\
-0.786 \\
-0.742 \\
-1.395 \\
-1.271 \\
\end{array}$ & $\begin{array}{l}5124.52 \\
5140.83 \\
5151.52\end{array}$ & $\begin{array}{l}\text { blend } \\
\text { blend } \\
\text { J78, lab } \\
\text { blend } \\
\text { blend, wrong? } \\
\text { blend, computed too strong } \\
\text { wrong, not observed } \\
\text { at the continuum level } \\
\text { at the level of the noise }\end{array}$ \\
\hline
\end{tabular}


A\&A 520, A57 (2010)

Table 7. continued.

\begin{tabular}{|c|c|c|c|c|c|c|c|}
\hline \multicolumn{2}{|l|}{ Upper level } & \multicolumn{2}{|c|}{ Lower level } & \multirow{2}{*}{$\begin{array}{c}\lambda(\text { calc }) \\
\AA\end{array}$} & \multirow{2}{*}{$\begin{array}{c}\log g f \\
\text { KUR }\end{array}$} & \multirow{2}{*}{$\begin{array}{c}\lambda(\text { obs }) \\
\AA\end{array}$} & \multirow[t]{2}{*}{ Notes } \\
\hline $\mathrm{cm}^{-1}$ & $J$ & $\mathrm{~cm}^{-1}$ & & & & & \\
\hline 123430.181 cont. & & $\begin{array}{l}105379.430 \\
105414.180 \\
105630.750 \\
106846.650 \\
106866.760 \\
107407.800 \\
110428.280 \\
110609.540\end{array}$ & $\begin{array}{l}\left({ }^{3} F\right) 4 d^{4} D_{5 / 2} \\
\left({ }^{3} F\right) 4 d^{4} G_{5 / 2} \\
\left({ }^{5} D\right) 5 d^{4} G_{5 / 2} \\
\left({ }^{3} F\right) 4 d^{4} F_{3 / 2} \\
\left({ }^{3} F\right) 4 d d^{4} F_{5 / 2} \\
\left({ }^{3} F\right) 4 d^{2} D_{5 / 2} \\
\left({ }^{3} G\right) 4 d{ }^{4} F_{5 / 2} \\
\left({ }^{3} G\right) 4 d{ }^{4} F_{3 / 2}\end{array}$ & $\begin{array}{l}5538.397 \\
5549.080 \\
5616.598 \\
6028.409 \\
6035.729 \\
6239.544 \\
7689.067 \\
7797.776\end{array}$ & $\begin{array}{l}-1.442 \\
-0.905 \\
-1.451 \\
-1.085 \\
-1.269 \\
-1.446 \\
-1.409 \\
-1.406\end{array}$ & 6028.40 & $\begin{array}{l}\text { at the level of the noise } \\
\text { blend } \\
\text { blend } \\
\text { at the level of the noise }\end{array}$ \\
\hline
\end{tabular}


F. Castelli and R. L. Kurucz: New Fe II energy levels from stellar spectra

Table 8. Fe II lines in the $3800-8000 \AA$ A region with $\log g f \geq-1.5$ and $3 \mathrm{~d}^{6}\left({ }^{3} \mathrm{~F}\right) 4 \mathrm{f}$ energy levels as upper levels.

\begin{tabular}{|c|c|c|c|c|c|c|c|c|c|}
\hline \multicolumn{4}{|c|}{ Upper level } & \multicolumn{2}{|c|}{ Lower level } & \multirow{2}{*}{$\begin{array}{c}\lambda(\text { calc }) \\
\AA\end{array}$} & \multirow{2}{*}{$\begin{array}{c}\log g f \\
\text { KUR }\end{array}$} & \multirow{2}{*}{$\begin{array}{c}\lambda(\mathrm{obs}) \\
\AA \\
\end{array}$} & \multirow[t]{2}{*}{ Notes } \\
\hline $\mathrm{cm}^{-1}$ & & & $J$ & $\mathrm{~cm}^{-1}$ & & & & & \\
\hline 124421.468 & $\left({ }^{3} F\right) 4 f$ & $4[7]$ & $15 / 2$ & $\begin{array}{l}103617.580 \\
104064.670 \\
104119.710 \\
104622.300 \\
105288.847\end{array}$ & $\begin{array}{l}\left({ }^{3} \mathrm{H}\right) 4 \mathrm{~d}^{4} \mathrm{H}_{13 / 2} \\
\left({ }^{3} \mathrm{H}\right) 4 \mathrm{~d}^{4} \mathrm{I}_{13 / 2} \\
\left({ }^{3} \mathrm{H}\right) 4 \mathrm{~d}^{2} \mathrm{~K}_{15 / 2} \\
\left({ }^{3} \mathrm{H}\right) 4 \mathrm{~d}^{2} \mathrm{I}_{13 / 2} \\
\left({ }^{3} \mathrm{~F}\right) 4 \mathrm{~d}^{4} \mathrm{H}_{13 / 2}\end{array}$ & $\begin{array}{l}4805.451 \\
4910.993 \\
4924.307 \\
5049.309 \\
5225.221\end{array}$ & $\begin{array}{l}-0.972 \\
-1.090 \\
-1.174 \\
-1.258 \\
+0.974\end{array}$ & $\begin{array}{l}4805.42 \\
\\
5049.3 \\
5225.229\end{array}$ & $\begin{array}{l}\text { at the continuum level } \\
\text { not obs } \\
\text { very weak } \\
\text { lab, J78 }\end{array}$ \\
\hline 124436.436 & $\left({ }^{3} F\right) 4 f$ & $4[7]$ & $13 / 2$ & $\begin{array}{l}103600.430 \\
103751.660 \\
104315.370 \\
104765.450 \\
105063.550 \\
105288.847 \\
105398.852 \\
105763.270 \\
106045.690 \\
108630.429\end{array}$ & $\begin{array}{l}\left({ }^{3} \mathrm{H}\right) 4 \mathrm{~d}^{4} \mathrm{G}_{11 / 2} \\
\left({ }^{3} \mathrm{H}\right) 4 \mathrm{~d}^{4} \mathrm{H}_{11 / 2} \\
\left({ }^{3} \mathrm{H}\right) 4 \mathrm{~d}^{2} \mathrm{~K}_{13 / 2} \\
\left({ }^{3} \mathrm{H}\right) 4 \mathrm{~d}^{2} \mathrm{I}_{11 / 2} \\
\left({ }^{3} \mathrm{~F}\right) 4 \mathrm{~d}^{4} \mathrm{G}_{11 / 2} \\
\left({ }^{3} \mathrm{~F}\right) 4 d^{4} \mathrm{H}_{13 / 2} \\
\left({ }^{3} \mathrm{~F}\right) 4 d^{4} \mathrm{H}_{11 / 2} \\
\left({ }^{3} \mathrm{~F}\right) 4 d^{2} \mathrm{H}_{11 / 2} \\
\left({ }^{3} \mathrm{H}\right) 4 \mathrm{~d}^{2} \mathrm{H}_{11 / 2} \\
\left({ }^{1} \mathrm{I}\right) 5 \mathrm{~s} \mathrm{e}^{2} \mathrm{I}_{11 / 2}\end{array}$ & $\begin{array}{l}4798.043 \\
4833.123 \\
4968.529 \\
5082.213 \\
5160.416 \\
5221.136 \\
5251.306 \\
5353.789 \\
5436.006 \\
6324.960\end{array}$ & $\begin{array}{l}-1.190 \\
-1.441 \\
-1.078 \\
-1.265 \\
-0.003 \\
-0.831 \\
+0.664 \\
+0.076 \\
-0.154 \\
-1.433\end{array}$ & $\begin{array}{l}4968.53 \\
5160.4 \\
5251.321 \\
5353.80 \\
5436.12\end{array}$ & $\begin{array}{l}\text { at the continuum level } \\
\text { very weak } \\
\text { blend } \\
\text { lab } \\
\text { blend, weak component } \\
\text { blend } \\
\text { at the continuum level }\end{array}$ \\
\hline 124400.107 & $\left({ }^{3} F\right) 4 f$ & $4[6]$ & $13 / 2$ & $\begin{array}{l}103600.430 \\
104174.270 \\
104765.450 \\
105063.550 \\
105288.850 \\
105398.850 \\
105763.270 \\
106045.690\end{array}$ & $\begin{array}{l}\left({ }^{3} \mathrm{H}\right) 4 \mathrm{~d}^{4} \mathrm{G}_{11 / 2} \\
\left({ }^{3} \mathrm{H}\right) 4 \mathrm{~d}^{4} \mathrm{I}_{11 / 2} \\
\left({ }^{3} \mathrm{H}\right) 4 \mathrm{~d}^{2} \mathrm{I}_{11 / 2} \\
\left({ }^{3} \mathrm{~F}\right) 4 \mathrm{~d}^{4} \mathrm{G}_{11 / 2} \\
\left({ }^{3} \mathrm{~F}\right) 4 \mathrm{~d}^{4} \mathrm{H}_{13 / 2} \\
\left({ }^{3} \mathrm{~F}\right) 4 \mathrm{~d}^{4} \mathrm{H}_{11 / 2} \\
\left({ }^{3} \mathrm{~F}\right) 4 \mathrm{~d}^{2} \mathrm{H}_{11 / 2} \\
\left({ }^{3} \mathrm{H}\right) 4 \mathrm{~d}^{2} \mathrm{H}_{11 / 2}\end{array}$ & $\begin{array}{l}4806.424 \\
4942.792 \\
5091.616 \\
5170.111 \\
5231.062 \\
5261.345 \\
5364.226 \\
5446.766\end{array}$ & $\begin{array}{l}-0.542 \\
-1.458 \\
-0.517 \\
+0.742 \\
+0.278 \\
+0.080 \\
-0.538 \\
-0.314\end{array}$ & $\begin{array}{l}4806.4 \\
5091.6 \\
5170.10 \\
5231.067 \\
5261.339 \\
5364.22 \\
5446.75\end{array}$ & $\begin{array}{l}\text { very weak } \\
\text { J78,lab, blended } \\
\text { lab } \\
\text { shifted? } \\
\text { blend }\end{array}$ \\
\hline 124402.557 & $\left({ }^{3} \mathrm{~F}\right) 4 \mathrm{f}$ & $4[6]$ & $11 / 2$ & $\begin{array}{l}103683.070 \\
104765.450 \\
104807.210 \\
104916.550 \\
105063.550 \\
105155.090 \\
105211.062 \\
105398.852 \\
105524.461 \\
105763.270 \\
106018.643 \\
106045.690 \\
106097.520 \\
106722.170 \\
106924.430 \\
109925.200 \\
110008.300\end{array}$ & $\begin{array}{l}\left({ }^{5} \mathrm{D}\right) 5 \mathrm{~d}^{4} \mathrm{~F}_{9 / 2} \\
\left({ }^{3} \mathrm{H}\right) 4 \mathrm{~d}^{2} \mathrm{I}_{11 / 2} \\
\left({ }^{3} \mathrm{H}\right) 4 \mathrm{~d}^{2} \mathrm{G}_{9 / 2} \\
\left({ }^{3} \mathrm{H}\right) 4 \mathrm{~d}^{4} \mathrm{~F}_{9 / 2} \\
\left({ }^{3} \mathrm{~F}\right) 4 \mathrm{~d}^{4} \mathrm{G}_{11 / 2} \\
\left({ }^{3} \mathrm{~F}\right) 4 \mathrm{~d}^{4} \mathrm{G}_{9 / 2} \\
\left({ }^{5} \mathrm{D}\right) 5 \mathrm{~d}^{4} \mathrm{G}_{9 / 2} \\
\left({ }^{3} \mathrm{~F}\right) 4 \mathrm{~d}^{4} \mathrm{H}_{11 / 2} \\
\left({ }^{3} \mathrm{~F}\right) 4 \mathrm{~d}^{4} \mathrm{H}_{9 / 2} \\
\left({ }^{3} \mathrm{~F}\right) 4 \mathrm{~d}^{2} \mathrm{H}_{11 / 2} \\
\left({ }^{3} \mathrm{~F}\right) 4 \mathrm{~d}^{2} \mathrm{H}_{9 / 2} \\
\left({ }^{3} \mathrm{H}\right) 4 \mathrm{~d}^{2} \mathrm{H}_{11 / 2} \\
\left({ }^{3} \mathrm{H}\right) 4 \mathrm{~d}^{2} \mathrm{H}_{9 / 2} \\
\left({ }^{3} \mathrm{~F}\right) 4 \mathrm{~d}^{4} \mathrm{~F}_{9 / 2} \\
\left({ }^{3} \mathrm{~F}\right) 4 \mathrm{~d}^{2} \mathrm{G}_{9 / 2} \\
\left({ }^{3} \mathrm{G}\right) 4 \mathrm{~d}^{2} \mathrm{G}_{9 / 2} \\
\left({ }^{3} \mathrm{G}\right) 4 \mathrm{~d}^{2} \mathrm{H}_{9 / 2}\end{array}$ & $\begin{array}{l}4825.028 \\
5090.983 \\
5101.830 \\
5130.460 \\
5169.456 \\
5194.042 \\
5209.193 \\
5260.668 \\
5295.671 \\
5363.520 \\
5438.027 \\
5446.039 \\
5461.459 \\
5654.418 \\
5719.850 \\
6765.246 \\
6945.303\end{array}$ & $\begin{array}{l}-1.407 \\
-1.256 \\
-1.382 \\
+0.158 \\
-0.871 \\
-0.084 \\
-0.494 \\
-0.049 \\
-1.274 \\
-0.269 \\
-0.914 \\
-0.626 \\
+0.179 \\
-0.044 \\
+0.097 \\
-1.049 \\
-1.190\end{array}$ & $\begin{array}{l}5101.82 \\
\\
5194.047 \\
5209.199 \\
5260.682 \\
5295.662 \\
5363.51 \\
\\
5446.05 \\
5461.48 \\
5719.85\end{array}$ & $\begin{array}{l}\text { computed too weak } \\
\text { blend } \\
\text { computed too strong } \\
\text { lab,J78 }\end{array}$ \\
\hline 124388.840 & $\left({ }^{3} \mathrm{~F}\right) 4 \mathrm{f}$ & $4[5]$ & $11 / 2$ & $\begin{array}{l}103600.430 \\
103683.070 \\
103771.320 \\
104765.450 \\
104807.210 \\
104868.500 \\
104916.550 \\
105063.550 \\
105155.090 \\
105211.062 \\
105288.847 \\
105398.852 \\
106045.690 \\
106722.170 \\
106924.430 \\
109811.920 \\
\end{array}$ & $\begin{array}{l}\left({ }^{3} \mathrm{H}\right) 4 \mathrm{~d}^{4} \mathrm{G}_{11 / 2} \\
\left({ }^{5} \mathrm{D}\right) 5 \mathrm{~d}^{4} \mathrm{~F}_{9 / 2} \\
\left({ }^{3} \mathrm{H}\right) 4 \mathrm{~d}^{4} \mathrm{G}_{9 / 2} \\
\left({ }^{3} \mathrm{H}\right) 4 \mathrm{~d}^{2} \mathrm{I}_{11 / 2} \\
\left({ }^{3} \mathrm{H}\right) 4 \mathrm{~d}^{2} \mathrm{G}_{9 / 2} \\
\left({ }^{5} \mathrm{D}\right) 5 \mathrm{~d}^{6} \mathrm{G}_{9 / 2} \\
\left({ }^{3} \mathrm{H}\right) 4 \mathrm{~d}^{4} \mathrm{~F}_{9 / 2} \\
\left({ }^{3} \mathrm{~F}\right) 4 \mathrm{~d}^{4} \mathrm{G}_{11 / 2} \\
\left({ }^{3} \mathrm{~F}\right) 4 \mathrm{~d}^{4} \mathrm{G}_{9 / 2} \\
\left({ }^{5} \mathrm{D}\right) 5 \mathrm{~d}^{4} \mathrm{G}_{9 / 2} \\
\left({ }^{3} \mathrm{~F}\right) 4 \mathrm{~d}^{4} \mathrm{H}_{13 / 2} \\
\left({ }^{3} \mathrm{~F}\right) 4 \mathrm{~d}^{4} \mathrm{H}_{11 / 2} \\
\left({ }^{3} \mathrm{H}\right) 4 \mathrm{~d}^{2} \mathrm{H}_{11 / 2} \\
\left({ }^{3} \mathrm{~F}\right) 4 \mathrm{~d}^{4} \mathrm{~F}_{9 / 2} \\
\left({ }^{3} \mathrm{~F}\right) 4 \mathrm{~d}^{2} \mathrm{G}_{9 / 2} \\
\left({ }^{3} \mathrm{G}\right) 4 \mathrm{~d}^{4} \mathrm{~F}_{9 / 2}\end{array}$ & $\begin{array}{l}4809.029 \\
4828.222 \\
4848.889 \\
5094.540 \\
5105.404 \\
5121.435 \\
5134.072 \\
5173.126 \\
5197.747 \\
5212.916 \\
5234.147 \\
5264.468 \\
5450.112 \\
5658.806 \\
5724.343 \\
6858.267 . \\
\end{array}$ & $\begin{array}{l}-0.852 \\
-0.829 \\
-0.699 \\
-0.517 \\
+0.158 \\
-0.968 \\
-0.161 \\
+0.425 \\
-0.166 \\
-0.199 \\
-0.630 \\
-0.717 \\
-1.282 \\
-0.643 \\
-0.429 \\
-0.903 \\
\end{array}$ & $\begin{array}{l}4809.02 \\
\\
5094.55 \\
5105.4 \\
5121.45 \\
\\
5173.12 \\
5197.756\end{array}$ & $\begin{array}{l}\text { weak, on the } \mathrm{H}_{\beta} \text { wing } \\
\text { lab } \\
\text { weak } \\
\text { blend } \\
\text { lab } \\
\text { blend } \\
\text { blend } \\
\text { blend } \\
\text { blend } \\
\text { blend, computed too strong } \\
\text { at the continuum level }\end{array}$ \\
\hline
\end{tabular}


Table 8. continued.

\begin{tabular}{|c|c|c|c|c|c|c|c|c|c|}
\hline \multicolumn{4}{|c|}{ Upper level } & \multicolumn{2}{|c|}{ Lower level } & \multirow{2}{*}{$\begin{array}{c}\lambda(\text { calc }) \\
\AA\end{array}$} & \multirow{2}{*}{$\begin{array}{c}\log g f \\
\text { KUR }\end{array}$} & \multirow{2}{*}{$\begin{array}{c}\lambda(\mathrm{obs}) \\
\AA\end{array}$} & \multirow[t]{2}{*}{ Notes } \\
\hline $\mathrm{cm}^{-1}$ & & & $J$ & $\mathrm{~cm}^{-1}$ & & & & & \\
\hline 124385.706 & $\left({ }^{3} \mathrm{~F}\right) 4 \mathrm{f}$ & $4[5]$ & $9 / 2$ & $\begin{array}{l}103771.320 \\
103986.330 \\
104807.210 \\
104993.860 \\
105063.550 \\
105123.000 \\
105155.090 \\
105211.062 \\
105220.600 \\
105291.010 \\
105398.852 \\
105775.491 \\
106018.640 \\
106097.520 \\
106722.170 \\
106767.210 \\
106900.370 \\
106924.430 \\
110167.280 \\
110570.300\end{array}$ & $\begin{array}{l}\left({ }^{3} \mathrm{H}\right) 4 d^{4} \mathrm{G}_{9 / 2} \\
\left({ }^{3} \mathrm{H}\right) 4 \mathrm{~d}^{4} \mathrm{H}_{7 / 2} \\
\left({ }^{3} \mathrm{H}\right) 4 \mathrm{~d}^{2} \mathrm{G}_{9 / 2} \\
\left({ }^{3} \mathrm{~F}\right) 4 \mathrm{~d}^{4} \mathrm{D}_{7 / 2} \\
\left({ }^{3} \mathrm{~F}\right) 4 \mathrm{~d}^{4} \mathrm{G}_{11 / 2} \\
\left({ }^{3} \mathrm{H}\right) 4 \mathrm{~d}^{2} \mathrm{G}_{7 / 2} \\
\left({ }^{3} \mathrm{~F}\right) 4 \mathrm{~d}^{4} \mathrm{G}_{9 / 2} \\
\left({ }^{5} \mathrm{D}\right) 5 \mathrm{~d}^{4} \mathrm{G}_{9 / 2} \\
\left({ }^{3} \mathrm{H}\right) 4 \mathrm{~d}^{4} \mathrm{~F}_{7 / 2} \\
\left({ }^{3} \mathrm{~F}\right) 4 \mathrm{~d}^{4} \mathrm{G}_{7 / 2} \\
\left({ }^{3} \mathrm{~F}\right) 4 \mathrm{~d}^{4} \mathrm{H}_{11 / 2} \\
\left({ }^{3} \mathrm{~F}\right) 4 \mathrm{~d}^{2} \mathrm{~F}_{7 / 2} \\
\left({ }^{3} \mathrm{~F}\right) 4 \mathrm{~d}^{2} \mathrm{H}_{9 / 2} \\
\left({ }^{3} \mathrm{H}\right) 4 \mathrm{~d}^{2} \mathrm{H}_{9 / 2} \\
\left({ }^{3} \mathrm{~F}\right) 4 \mathrm{~d}^{4} \mathrm{~F}_{9 / 2} \\
\left({ }^{3} \mathrm{~F}\right) 4 \mathrm{~d}^{4} \mathrm{~F}_{7 / 2} \\
\left({ }^{3} \mathrm{~F}\right) 4 \mathrm{~d}^{2} \mathrm{G}_{7 / 2} \\
\left({ }^{3} \mathrm{~F}\right) 4 \mathrm{~d}^{2} \mathrm{G}_{9 / 2} \\
\left({ }^{3} \mathrm{G}\right) 4 \mathrm{~d}^{4} \mathrm{~F}_{7 / 2} \\
\left({ }^{3} \mathrm{G}\right) 4 \mathrm{~d}^{2} \mathrm{~F}_{7 / 2}\end{array}$ & $\begin{array}{l}4849.626 \\
4900.742 \\
5106.222 \\
5155.371 \\
5173.965 \\
5189.933 \\
5198.594 \\
5213.769 \\
5216.634 \\
5235.599 \\
5265.337 \\
5371.899 \\
5443.015 \\
5466.492 \\
5659.810 \\
5674.279 \\
5717.492 \\
5725.370 \\
7031.188 \\
7236.302\end{array}$ & $\begin{array}{l}-1.159 \\
-1.404 \\
-0.305 \\
-0.195 \\
-0.955 \\
-0.112 \\
-0.154 \\
-0.389 \\
-1.420 \\
-0.769 \\
-0.986 \\
+0.199 \\
-1.240 \\
-0.492 \\
-1.436 \\
-1.037 \\
-1.080 \\
-0.147 \\
-1.480 \\
-1.125\end{array}$ & $\begin{array}{l}5155.37 \\
5173.98 \\
5198.596 \\
5213.78 \\
\\
5265.323 \\
5371.90 \\
5466.49 \\
5674.30 \\
5725.35\end{array}$ & $\begin{array}{l}\mathrm{H}_{\beta} \text { wing, not obs. } \\
\text { at the continuum level } \\
\text { computed too strong } \\
\text { computed too weak } \\
\text { blend }\end{array}$ \\
\hline 124401.939 & $\left({ }^{3} \mathrm{~F}\right) 4 \mathrm{f}$ & $4[4]$ & $9 / 2$ & $\begin{array}{l}103683.070 \\
103771.320 \\
104481.590 \\
104765.450 \\
104807.210 \\
104868.500 \\
104916.550 \\
104993.860 \\
105063.550 \\
105155.090 \\
105211.062 \\
105220.600 \\
105291.010 \\
105763.270 \\
105775.491 \\
106097.520 \\
106722.170 \\
106900.370 \\
109811.920\end{array}$ & $\begin{array}{l}\left({ }^{5} \mathrm{D}\right) 5 \mathrm{~d}^{4} \mathrm{~F}_{9 / 2} \\
\left({ }^{3} \mathrm{H}\right) 4 \mathrm{~d}^{4} \mathrm{G}_{9 / 2} \\
\left({ }^{3} \mathrm{H}\right) 4 \mathrm{~d}^{2} \mathrm{~F}_{7 / 2} \\
\left({ }^{3} \mathrm{H}\right) 4 \mathrm{~d}^{2} \mathrm{I}_{11 / 2} \\
\left({ }^{3} \mathrm{H}\right) 4 \mathrm{~d}^{2} \mathrm{G}_{9 / 2} \\
\left({ }^{5} \mathrm{D}\right) 5 \mathrm{~d}^{6} \mathrm{G}_{9 / 2} \\
\left({ }^{3} \mathrm{H}\right) 4 \mathrm{~d}^{4} \mathrm{~F}_{9 / 2} \\
\left({ }^{3} \mathrm{~F}\right) 4 \mathrm{~d}^{4} \mathrm{D}_{7 / 2} \\
\left({ }^{3} \mathrm{~F}\right) 4 \mathrm{~d}^{4} \mathrm{G}_{11 / 2} \\
\left({ }^{3} \mathrm{~F}\right) 4 \mathrm{~d}^{4} \mathrm{G}_{9 / 2} \\
\left({ }^{5} \mathrm{D}\right) 5 \mathrm{~d}^{4} \mathrm{G}_{9 / 2} \\
\left({ }^{3} \mathrm{H}\right) 4 \mathrm{~d}^{4} \mathrm{~F}_{7 / 2} \\
\left({ }^{3} \mathrm{~F}\right) 4 \mathrm{~d}^{4} \mathrm{G}_{7 / 2} \\
\left({ }^{3} \mathrm{~F}\right) 4 \mathrm{~d}^{2} \mathrm{H}_{11 / 2} \\
\left({ }^{3} \mathrm{~F}\right) 4 \mathrm{~d}^{2} \mathrm{~F}_{7 / 2} \\
\left({ }^{3} \mathrm{H}\right) 4 \mathrm{~d}^{2} \mathrm{H}_{9 / 2} \\
\left({ }^{3} \mathrm{~F}\right) 4 \mathrm{~d}^{4} \mathrm{~F}_{9 / 2} \\
\left({ }^{3} \mathrm{~F}\right) 4 \mathrm{~d}^{2} \mathrm{G}_{7 / 2} \\
\left({ }^{3} \mathrm{G}\right) 4 \mathrm{~d}^{4} \mathrm{~F}_{9 / 2}\end{array}$ & $\begin{array}{l}4825.170 \\
4845.810 \\
5018.593 \\
5091.141 \\
5101.991 \\
5118.000 \\
5130.621 \\
5151.058 \\
5169.622 \\
5194.209 \\
5209.359 \\
5211.949 \\
5231.152 \\
5363.698 \\
5367.218 \\
5461.644 \\
5654.613 \\
5712.189 \\
6852.110\end{array}$ & $\begin{array}{l}-0.851 \\
-1.216 \\
-0.782 \\
-1.199 \\
-0.285 \\
-0.871 \\
+0.114 \\
-0.280 \\
-0.361 \\
-1.245 \\
-1.260 \\
+0.055 \\
-0.836 \\
-1.391 \\
-0.182 \\
-0.455 \\
-0.197 \\
-1.361 \\
-0.955\end{array}$ & $\begin{array}{l}5091.15 \\
\\
5117.98 \\
5130.60 \\
5151.07 \\
5169.6\end{array}$ & $\begin{array}{l}\text { on the } \mathrm{H}_{\beta} \text { wing } \\
\text { blend Fe II } 5018.440 \\
\text { wrong,not observed } \\
\text { lab } \\
\text { lab } \\
\text { blend Fe III } \\
\text { lab } \\
\text { blend } \\
\text { blend }\end{array}$ \\
\hline 124385.010 & $\left({ }^{3} \mathrm{~F}\right) 4 \mathrm{f}$ & $4[4]$ & $7 / 2$ & $\begin{array}{l}103191.917 \\
103597.402 \\
104807.210 \\
104993.860 \\
105123.000 \\
105155.090 \\
105211.062 \\
105220.600 \\
105234.237 \\
105291.010 \\
105775.836 \\
106097.520 \\
106208.560 \\
106767.210 \\
106796.660 \\
106866.760 \\
106900.370\end{array}$ & $\begin{array}{l}\left({ }^{3} \mathrm{P}\right) 4 \mathrm{~d}^{2} \mathrm{~F}_{7 / 2} \\
\left({ }^{3} \mathrm{P}\right) 4 \mathrm{~d}^{2} \mathrm{D}_{5 / 2} \\
\left({ }^{3} \mathrm{H}\right) 4 \mathrm{~d}^{2} \mathrm{G}_{9 / 2} \\
\left({ }^{3} \mathrm{~F}\right) 4 \mathrm{~d}^{4} \mathrm{D}_{7 / 2} \\
\left({ }^{3} \mathrm{H}\right) 4 \mathrm{~d}^{2} \mathrm{G}_{7 / 2} \\
\left({ }^{3} \mathrm{~F}\right) 4 \mathrm{~d}^{4} \mathrm{G}_{9 / 2} \\
\left({ }^{5} \mathrm{D}\right) 5 \mathrm{~d}^{4} \mathrm{G}_{9 / 2} \\
\left({ }^{3} \mathrm{H}\right) 4 \mathrm{~d}^{4} \mathrm{~F}_{7 / 2} \\
\left({ }^{3} \mathrm{H}\right) 4 \mathrm{~d}^{4} \mathrm{~F}_{5 / 2} \\
\left({ }^{3} \mathrm{~F}\right) 4 \mathrm{~d}^{4} \mathrm{G}_{7 / 2} \\
\left({ }^{3} \mathrm{~F}\right) 4 \mathrm{~d}^{2} \mathrm{~F}_{7 / 2} \\
\left({ }^{3} \mathrm{H}\right) 4 d^{2} \mathrm{H}_{9 / 2} \\
\left({ }^{3} \mathrm{~F}\right) 4 \mathrm{~d}^{2} \mathrm{~F}_{5 / 2} \\
\left({ }^{3} \mathrm{~F}\right) 4 \mathrm{~d}^{4} \mathrm{~F}_{7 / 2} \\
\left({ }^{3} \mathrm{~F}\right) 4 \mathrm{~d}^{4} \mathrm{P}_{5 / 2} \\
\left({ }^{3} \mathrm{~F}\right) 4 \mathrm{~d}^{4} \mathrm{~F}_{5 / 2} \\
\left({ }^{3} \mathrm{~F}\right) 4 d^{2} \mathrm{G}_{7 / 2}\end{array}$ & $\begin{array}{l}4717.199 \\
4809.214 \\
5106.403 \\
5155.556 \\
5190.121 \\
5198.782 \\
5213.958 \\
5216.553 \\
5220.268 \\
5235.790 \\
5372.100 \\
5466.700 \\
5500.096 \\
5674.503 \\
5684.004 \\
5706.743 \\
5717.719\end{array}$ & $\begin{array}{l}-1.461 \\
-1.233 \\
-1.091 \\
-0.412 \\
-0.246 \\
-0.950 \\
-1.188 \\
-1.332 \\
-1.463 \\
-0.829 \\
+0.165 \\
-1.095 \\
-0.922 \\
-1.298 \\
-0.895 \\
-0.920 \\
-1.023 \\
\end{array}$ & $\begin{array}{l}5155.56 \\
5190.123\end{array}$ & $\begin{array}{l}\text { blend } \\
\text { blend } \\
\text { blend } \\
\text { blend } \\
\text { lab } \\
\text { at the level of the noise } \\
\text { blend } \\
\text { computed too weak }\end{array}$ \\
\hline
\end{tabular}


F. Castelli and R. L. Kurucz: New Fe II energy levels from stellar spectra

Table 8. continued.

\begin{tabular}{|c|c|c|c|c|c|c|c|c|c|}
\hline \multicolumn{4}{|c|}{ Upper level } & \multicolumn{2}{|c|}{ Lower level } & \multirow{2}{*}{$\begin{array}{c}\lambda(\mathrm{calc}) \\
\AA \\
\end{array}$} & \multirow{2}{*}{$\begin{array}{c}\log g f \\
\text { KUR }\end{array}$} & \multirow{2}{*}{$\begin{array}{c}\lambda(\mathrm{obs}) \\
\AA \\
\end{array}$} & \multirow[t]{2}{*}{ Notes } \\
\hline $\mathrm{cm}^{-1}$ & & & $J$ & $\mathrm{~cm}^{-1}$ & & & & & \\
\hline 124385.010 & cont. & & & $\begin{array}{l}106924.430 \\
107407.800 \\
110570.300\end{array}$ & $\begin{array}{l}\left({ }^{3} \mathrm{~F}\right) 4 \mathrm{~d}^{2} \mathrm{G}_{9 / 2} \\
\left({ }^{3} \mathrm{~F}\right) 4 \mathrm{~d}^{2} \mathrm{D}_{5 / 2} \\
\left({ }^{3} \mathrm{G}\right) 4 \mathrm{~d}^{2} \mathrm{~F}_{7 / 2}\end{array}$ & $\begin{array}{l}5725.598 \\
5888.617 \\
7236.667\end{array}$ & $\begin{array}{l}-0.824 \\
-0.044 \\
-1.221\end{array}$ & $\begin{array}{l}5725.60 \\
5888.61\end{array}$ & at the level of the noise \\
\hline 124416.110 & $\left({ }^{3} \mathrm{~F}\right) 4 \mathrm{f}$ & $4[3]$ & $7 / 2$ & $\begin{array}{l}103683.070 \\
104481.590 \\
104807.210 \\
104868.500 \\
104916.550 \\
104993.860 \\
105123.000 \\
105155.090 \\
105211.062 \\
105220.600 \\
105291.010 \\
105379.430 \\
105775.491 \\
106097.520 \\
106722.170 \\
106767.210 \\
106796.660 \\
107407.800 \\
109811.920\end{array}$ & $\begin{array}{l}\left({ }^{5} \mathrm{D}\right) 5 \mathrm{~d}^{4} \mathrm{~F}_{9 / 2} \\
\left({ }^{3} \mathrm{H}\right) 4 \mathrm{~d}^{2} \mathrm{~F}_{7 / 2} \\
\left({ }^{3} \mathrm{H}\right) 4 \mathrm{~d}^{2} \mathrm{G}_{9 / 2} \\
\left({ }^{5} \mathrm{D}\right) 5 \mathrm{~d}^{6} \mathrm{G}_{9 / 2} \\
\left({ }^{3} \mathrm{H}\right) 4 \mathrm{~d}^{4} \mathrm{~F}_{9 / 2} \\
\left({ }^{3} \mathrm{~F}\right) 4 \mathrm{~d}^{4} \mathrm{D}_{7 / 2} \\
\left({ }^{3} \mathrm{H}\right) 4 \mathrm{~d}^{2} \mathrm{G}_{7 / 2} \\
\left({ }^{3} \mathrm{~F}\right) 4 \mathrm{~d}^{4} \mathrm{G}_{9 / 2} \\
\left({ }^{5} \mathrm{D}\right) 5 \mathrm{~d}^{4} \mathrm{G}_{9 / 2} \\
\left({ }^{3} \mathrm{H}\right) 4 \mathrm{~d}^{4} \mathrm{~F}_{7 / 2} \\
\left({ }^{3} \mathrm{~F}\right) 4 \mathrm{~d}^{4} \mathrm{G}_{7 / 2} \\
\left({ }^{3} \mathrm{~F}\right) 4 \mathrm{~d}^{4} \mathrm{D}_{5 / 2} \\
\left({ }^{3} \mathrm{~F}\right) 4 \mathrm{~d}^{2} \mathrm{~F}_{7 / 2} \\
\left({ }^{3} \mathrm{H}\right) 4 \mathrm{~d}^{2} \mathrm{H}_{9 / 2} \\
\left({ }^{3} \mathrm{~F}\right) 4 \mathrm{~d}^{4} \mathrm{~F}_{9 / 2} \\
\left({ }^{3} \mathrm{~F}\right) 4 \mathrm{~d}^{4} \mathrm{~F}_{7 / 2} \\
\left({ }^{3} \mathrm{~F}\right) 4 \mathrm{~d}^{4} \mathrm{P}_{5 / 2} \\
\left({ }^{3} \mathrm{~F}\right) 4 \mathrm{~d}^{2} \mathrm{D}_{5 / 2} \\
\left({ }^{3} \mathrm{G}\right) 4 \mathrm{~d}^{4} \mathrm{~F}_{9 / 2}\end{array}$ & $\begin{array}{l}4821.172 \\
5015.025 \\
5098.304 \\
5114.290 \\
5126.892 \\
5147.300 \\
5181.754 \\
5190.388 \\
5205.515 \\
5208.101 \\
5227.276 \\
5251.555 \\
5363.137 \\
5457.419 \\
5650.084 \\
5664.504 \\
5673.972 \\
5877.850 \\
6845.461\end{array}$ & $\begin{array}{l}-1.273 \\
-0.607 \\
-0.623 \\
-1.355 \\
-0.477 \\
+0.051 \\
-1.028 \\
-1.077 \\
-1.184 \\
+0.031 \\
-1.201 \\
-1.289 \\
-0.687 \\
-1.335 \\
-0.819 \\
-1.029 \\
-0.486 \\
-1.281 \\
-1.364\end{array}$ & $\begin{array}{l}5015.02 \\
\\
5126.84 \\
5147.25 \\
5181.75 \\
\\
5208.99\end{array}$ & $\begin{array}{l}\text { computed too strong } \\
\text { lab, blend } \\
\text { blend,lab } \\
\text { computed too weak } \\
\text { blend } \\
\text { blend } \\
\text { blend } \\
\text { at the continuum level } \\
\text { blend } \\
\text { blend } \\
\text { at the level of the noise } \\
\text { blend } \\
\text { at the level of the noise } \\
\text { not observed }\end{array}$ \\
\hline 124403.474 & $\left({ }^{3} \mathrm{~F}\right) 4 \mathrm{f}$ & $4[3]$ & $5 / 2$ & $\begin{array}{l}103597.402 \\
104993.860 \\
105123.000 \\
105234.237 \\
105291.010 \\
105317.440 \\
105460.230 \\
105518.140 \\
105775.491 \\
106208.560 \\
106796.660 \\
106866.760 \\
107065.900 \\
107407.800 \\
107430.250 \\
108105.900 \\
110611.800\end{array}$ & $\begin{array}{l}\left({ }^{3} \mathrm{P}\right) 4 \mathrm{~d}^{2} \mathrm{D}_{5 / 2} \\
\left({ }^{3} \mathrm{~F}\right) 4 \mathrm{~d}^{4} \mathrm{D}_{7 / 2} \\
\left({ }^{3} \mathrm{H}\right) 4 \mathrm{~d}^{2} \mathrm{G}_{7 / 2} \\
\left({ }^{3} \mathrm{H}\right) 4 \mathrm{~d}^{4} \mathrm{~F}_{5 / 2} \\
\left({ }^{3} \mathrm{~F}\right) 4 \mathrm{~d}^{4} \mathrm{G}_{7 / 2} \\
\left({ }^{3} \mathrm{P}\right) 4 \mathrm{~d}^{2} \mathrm{P}_{3 / 2} \\
\left({ }^{3} \mathrm{~F}\right) 4 \mathrm{~d}^{4} \mathrm{D}_{3 / 2} \\
\left({ }^{3} \mathrm{H}\right) 4 \mathrm{~d}^{4} \mathrm{~F}_{3 / 2} \\
\left({ }^{3} \mathrm{~F}\right) 4 \mathrm{~d}^{2} \mathrm{~F}_{7 / 2} \\
\left({ }^{3} \mathrm{~F}\right) 4 \mathrm{~d}^{2} \mathrm{~F}_{5 / 2} \\
\left({ }^{3} \mathrm{~F}\right) 4 d^{4} \mathrm{P}_{5 / 2} \\
\left({ }^{3} \mathrm{~F}\right) 4 d^{4} \mathrm{~F}_{5 / 2} \\
\left({ }^{3} \mathrm{~F}\right) 4 d^{4} \mathrm{P}_{3 / 2} \\
\left({ }^{3} \mathrm{~F}\right) 4 d^{2} \mathrm{D}_{5 / 2} \\
\left({ }^{3} \mathrm{~F}\right) 4 d^{2} \mathrm{D}_{3 / 2} \\
\left({ }^{3} \mathrm{~F}\right) 4 d^{2} \mathrm{P}_{3 / 2} \\
\left({ }^{3} \mathrm{G}\right) 4 d^{2} \mathrm{~F}_{5 / 2}\end{array}$ & $\begin{array}{l}4804.946 \\
5150.651 \\
5185.150 \\
5215.240 \\
5230.732 \\
5237.975 \\
5277.458 \\
5293.641 \\
5366.775 \\
5494.515 \\
5678.044 \\
5700.741 \\
5766.220 \\
5882.220 \\
5890.000 \\
6134.185 \\
7248.754\end{array}$ & $\begin{array}{l}-1.146 \\
-0.855 \\
-0.746 \\
-1.455 \\
-1.416 \\
-1.304 \\
-0.778 \\
-1.294 \\
-0.450 \\
-0.721 \\
-1.006 \\
-0.790 \\
-1.192 \\
-0.040 \\
-0.918 \\
-0.702 \\
-1.434\end{array}$ & $\begin{array}{l}4804.93 \\
5185.141 \\
\\
\\
5293.627 \\
5366.78 \\
5494.51 \\
5700.76 \\
5882.22 \\
6134.2\end{array}$ & $\begin{array}{l}\text { computed too weak } \\
\text { lab,blend } \\
\text { blend } \\
\text { blend } \\
\text { blend } \\
\text { wrong, not observed } \\
\text { computed too low? } \\
\text { computed too strong } \\
\text { at the level of the noise } \\
\text { blend Na I } \\
\text { blend with telluric }\end{array}$ \\
\hline 124434.563 & $\left({ }^{3} \mathrm{~F}\right) 4 \mathrm{f}$ & $4[2]$ & $5 / 2$ & $\begin{array}{l}103597.402 \\
104120.270 \\
104209.610 \\
104481.590 \\
104993.860 \\
105213.000 \\
105127.770 \\
105220.600 \\
105379.430 \\
105775.491 \\
106208.560 \\
106767.210 \\
106796.660 \\
106866.760 \\
107407.800\end{array}$ & $\begin{array}{l}\left({ }^{3} \mathrm{P}\right) 4 \mathrm{~d}^{2} \mathrm{D}_{5 / 2} \\
\left({ }^{5} \mathrm{D}\right) 5 \mathrm{~d}^{6} \mathrm{P}_{5 / 2} \\
\left({ }^{3} \mathrm{H}\right) 4 \mathrm{~d}^{2} \mathrm{~F}_{5 / 2} \\
\left({ }^{3} \mathrm{H}\right) 4 \mathrm{~d}^{2} \mathrm{~F}_{7 / 2} \\
\left({ }^{3} \mathrm{~F}\right) 4 \mathrm{~d}^{4} \mathrm{D}_{7 / 2} \\
\left({ }^{3} \mathrm{H}\right) 4 \mathrm{~d}^{2} \mathrm{G}_{7 / 2} \\
\left({ }^{5} \mathrm{D}\right) 5 \mathrm{~d}^{4} \mathrm{D}_{5 / 2} \\
\left({ }^{3} \mathrm{H}\right) 4 \mathrm{~d}^{4} \mathrm{~F}_{7 / 2} \\
\left({ }^{3} \mathrm{~F}\right) 4 \mathrm{~d}^{4} \mathrm{D}_{5 / 2} \\
\left({ }^{3} \mathrm{~F}\right) 4 \mathrm{~d}^{2} \mathrm{~F}_{7 / 2} \\
\left({ }^{3} \mathrm{~F}\right) 4 \mathrm{~d}^{2} \mathrm{~F}_{5 / 2} \\
\left({ }^{3} \mathrm{~F}\right) 4 \mathrm{~d}^{4} \mathrm{~F}_{7 / 2} \\
\left({ }^{3} \mathrm{~F}\right) 4 \mathrm{~d}^{4} \mathrm{P}_{5 / 2} \\
\left({ }^{3} \mathrm{~F}\right) 4 \mathrm{~d}^{4} \mathrm{~F}_{5 / 2} \\
\left({ }^{3} \mathrm{~F}\right) 4 \mathrm{~d}^{2} \mathrm{D}_{5 / 2}\end{array}$ & $\begin{array}{l}4797.777 \\
4921.269 \\
4943.008 \\
5010.387 \\
5142.414 \\
5176.803 \\
5178.082 \\
5203.100 \\
5246.469 \\
5357.833 \\
5485.142 \\
5658.587 \\
5668.035 \\
5690.652 \\
5871.480\end{array}$ & $\begin{array}{l}-1.440 \\
-0.982 \\
-1.371 \\
-0.817 \\
-0.113 \\
-1.156 \\
-1.132 \\
-0.191 \\
-0.830 \\
-1.105 \\
-1.413 \\
-1.147 \\
-0.132 \\
-1.300 \\
-1.133\end{array}$ & $\begin{array}{l}4943.0 \\
5010.4 \\
5142.42 \\
\\
5178.08 \\
5203.10\end{array}$ & $\begin{array}{l}\text { blend } \\
\text { lab } \\
\text { blend } \\
\text { computed too weak } \\
\text { at the noise level, computed too strong } \\
\text { blend } \\
\text { computed too strong } \\
\text { computed too weak }\end{array}$ \\
\hline
\end{tabular}


Table 8. continued.

\begin{tabular}{|c|c|c|c|c|c|c|c|c|c|}
\hline \multicolumn{4}{|c|}{ Upper level } & \multicolumn{2}{|c|}{ Lower level } & \multirow{2}{*}{$\begin{array}{c}\lambda(\text { calc }) \\
\AA \\
\end{array}$} & \multirow{2}{*}{$\begin{array}{c}\log g f \\
\text { KUR }\end{array}$} & \multirow{2}{*}{$\begin{array}{c}\lambda(\mathrm{obs}) \\
\AA \\
\end{array}$} & \multirow[t]{2}{*}{ Notes } \\
\hline $\mathrm{cm}^{-1}$ & & & $J$ & $\mathrm{~cm}^{-1}$ & & & & & \\
\hline 124460.410 & $\left({ }^{3} F\right) 4 f$ & $4[2]$ & $3 / 2$ & $\begin{array}{l}104120.270 \\
104189.380 \\
105234.060 \\
105317.440 \\
105379.430 \\
105460.230 \\
105518.140 \\
106208.560 \\
106846.650 \\
106866.760 \\
107065.930 \\
107407.800 \\
107430.250 \\
108105.900\end{array}$ & $\begin{array}{l}\left({ }^{5} \mathrm{D}\right) 5 \mathrm{~d}{ }^{6} \mathrm{P}_{5 / 2} \\
\left({ }^{5} \mathrm{D}\right) 5 \mathrm{~d}^{4} \mathrm{P}_{3 / 2} \\
\left({ }^{3} \mathrm{H}\right) 4 \mathrm{~d}^{4} \mathrm{~F}_{5 / 2} \\
\left({ }^{3} \mathrm{P}\right) 4 \mathrm{~d}^{2} \mathrm{P}_{3 / 2} \\
\left({ }^{3} \mathrm{~F}\right) 4 \mathrm{~d}^{4} \mathrm{D}_{5 / 2} \\
\left({ }^{3} \mathrm{~F}\right) 4 \mathrm{~d}^{4} \mathrm{D}_{3 / 2} \\
\left({ }^{3} \mathrm{H}\right) 4 \mathrm{~d}^{4} \mathrm{~F}_{3 / 2} \\
\left({ }^{3} \mathrm{~F}\right) 4 \mathrm{~d}^{2} \mathrm{~F}_{5 / 2} \\
\left({ }^{3} \mathrm{~F}\right) 4 \mathrm{~d}^{4} \mathrm{~F}_{3 / 2} \\
\left({ }^{3} \mathrm{~F}\right) 4 \mathrm{~d}^{4} \mathrm{~F}_{5 / 2} \\
\left({ }^{3} \mathrm{~F}\right) 4 \mathrm{~d}^{4} \mathrm{P}_{3 / 2} \\
\left({ }^{3} \mathrm{~F}\right) 4 \mathrm{~d}^{2} \mathrm{D}_{5 / 2} \\
\left({ }^{3} \mathrm{~F}\right) 4 \mathrm{~d}^{2} \mathrm{D}_{3 / 2} \\
\left({ }^{3} \mathrm{~F}\right) 4 \mathrm{~d}^{2} \mathrm{P}_{3 / 2}\end{array}$ & $\begin{array}{l}4915.015 \\
4931.772 \\
5199.747 \\
5222.396 \\
5239.362 \\
5261.644 \\
5277.730 \\
5477.375 \\
5675.805 \\
5682.292 \\
5747.356 \\
5862.580 \\
5870.308 \\
6112.829\end{array}$ & $\begin{array}{l}-1.449 \\
-1.122 \\
-1.496 \\
-0.923 \\
-1.350 \\
-0.436 \\
-1.098 \\
-1.153 \\
-1.332 \\
-0.926 \\
-0.824 \\
-0.452 \\
-0.663 \\
-0.452\end{array}$ & $\begin{array}{l}5862.58 \\
5870.30\end{array}$ & $\begin{array}{l}\text { wrong,not observed } \\
\text { blend } \\
\text { blend } \\
\text { wrong, not observed } \\
\text { blend } \\
\text { at the level of the noise } \\
\text { at the level of the noise } \\
\text { at the level of the noise } \\
\text { at the level of the noise } \\
\text { at the level of thec noise } \\
\text { computed too weak } \\
\text { EMISSION? }\end{array}$ \\
\hline 124661.274 & $\left({ }^{3} \mathrm{~F}\right) 4 \mathrm{f}$ & $3[6]$ & $13 / 2$ & $\begin{array}{l}103751.660 \\
105063.550 \\
105398.852 \\
105763.270 \\
106045.690\end{array}$ & $\begin{array}{l}\left({ }^{3} \mathrm{H}\right) 4 \mathrm{~d}^{4} \mathrm{H}_{11 / 2} \\
\left({ }^{3} \mathrm{~F}\right) 4 \mathrm{~d}^{4} \mathrm{G}_{11 / 2} \\
\left({ }^{3} \mathrm{~F}\right) 4 \mathrm{~d}^{4} \mathrm{H}_{11 / 2} \\
\left({ }^{3} \mathrm{~F}\right) 4 \mathrm{~d}^{2} \mathrm{H}_{11 / 2} \\
\left({ }^{3} \mathrm{H}\right) 4 \mathrm{~d}^{2} \mathrm{H}_{11 / 2}\end{array}$ & $\begin{array}{l}4781.152 \\
5101.212 \\
5190.010 \\
5290.092 \\
5370.350\end{array}$ & $\begin{array}{l}-1.241 \\
-1.511 \\
+0.482 \\
+0.589 \\
+0.111\end{array}$ & $\begin{array}{l}4781.15 \\
5101.2 \\
5190.012 \\
5290.094 \\
5370.3\end{array}$ & $\begin{array}{l}\text { computed too weak } \\
\text { computed too weak } \\
\text { Fe II,5270.284 main comp. }\end{array}$ \\
\hline 124656.535 & $\left({ }^{3} F\right) 4 f$ & $3[6]$ & $11 / 2$ & $\begin{array}{l}103874.260 \\
104192.480 \\
105155.090 \\
105398.852 \\
105524.461 \\
105763.270 \\
106018.643 \\
106722.170 \\
106924.430 \\
109625.200\end{array}$ & $\begin{array}{l}\left({ }^{3} \mathrm{H}\right) 4 d^{4} \mathrm{H}_{9 / 2} \\
\left({ }^{3} \mathrm{H}\right) 4 \mathrm{~d}^{4} \mathrm{I}_{9 / 2} \\
\left({ }^{3} \mathrm{~F}\right) 4 \mathrm{~d}^{4} \mathrm{G}_{9 / 2} \\
\left({ }^{3} \mathrm{~F}\right) 4 \mathrm{~d}^{4} \mathrm{H}_{11 / 2} \\
\left({ }^{3} \mathrm{~F}\right) 4 \mathrm{~d}^{4} \mathrm{H}_{9 / 2} \\
\left({ }^{3} \mathrm{~F}\right) 4 \mathrm{~d}^{2} \mathrm{H}_{11 / 2} \\
\left({ }^{3} \mathrm{~F}\right) 4 \mathrm{~d}^{2} \mathrm{H}_{9 / 2} \\
\left({ }^{3} \mathrm{~F}\right) 4 \mathrm{~d}^{4} \mathrm{~F}_{9 / 2} \\
\left({ }^{3} \mathrm{~F}\right) 4 \mathrm{~d}^{2} \mathrm{G}_{9 / 2} \\
\left({ }^{3} \mathrm{G}\right) 4 \mathrm{~d}^{2} \mathrm{G}_{9 / 2}\end{array}$ & $\begin{array}{l}4810.449 \\
4885.254 \\
5126.398 \\
5191.288 \\
5225.371 \\
5291.420 \\
5363.923 \\
5574.341 \\
5637.925 \\
6650.935\end{array}$ & $\begin{array}{l}-1.268 \\
-1.238 \\
-0.847 \\
-1.025 \\
+0.768 \\
-1.047 \\
+0.201 \\
-1.111 \\
-0.160 \\
-1.387\end{array}$ & $\begin{array}{l}5225.364 \\
5363.92 \\
5574.25 \\
5637.92\end{array}$ & $\begin{array}{l}\text { weak } \\
\text { blend } \\
\text { very weak } \\
\text { blend } \\
\text { lab + unid } \\
\text { very weak } \\
\text { lab } \\
\text { blend }\end{array}$ \\
\hline 124626.900 & $\left({ }^{3} F\right) 4 f$ & $3[5]$ & $11 / 2$ & $\begin{array}{l}103683.070 \\
103771.320 \\
104807.210 \\
104916.550 \\
105063.550 \\
105155.090 \\
105211.062 \\
105398.852 \\
105524.461 \\
105763.270 \\
106018.643 \\
106045.690 \\
106097.520 \\
106722.170 \\
106924.430 \\
109811.920\end{array}$ & $\begin{array}{l}\left({ }^{5} \mathrm{D}\right) 5 \mathrm{~d}^{4} \mathrm{~F}_{9 / 2} \\
\left({ }^{3} \mathrm{H}\right) 4 \mathrm{~d}^{4} \mathrm{G}_{9 / 2} \\
\left({ }^{3} \mathrm{H}\right) 4 \mathrm{~d}^{2} \mathrm{G}_{9 / 2} \\
\left({ }^{3} \mathrm{H}\right) 4 \mathrm{~d}^{4} \mathrm{~F}_{9 / 2} \\
\left({ }^{3} \mathrm{~F}\right) 4 \mathrm{~d}^{4} \mathrm{G}_{11 / 2} \\
\left({ }^{3} \mathrm{~F}\right) 4 \mathrm{~d}^{4} \mathrm{G}_{9 / 2} \\
\left({ }^{5} \mathrm{D}\right) 5 \mathrm{~d}^{4} \mathrm{G}_{9 / 2} \\
\left({ }^{3} \mathrm{~F}\right) 4 \mathrm{~d}^{4} \mathrm{H}_{11 / 2} \\
\left({ }^{3} \mathrm{~F}\right) 4 \mathrm{~d}^{4} \mathrm{H}_{9 / 2} \\
\left({ }^{3} \mathrm{~F}\right) 4 \mathrm{~d}^{2} \mathrm{H}_{11 / 2} \\
\left({ }^{3} \mathrm{~F}\right) 4 \mathrm{~d}^{2} \mathrm{H}_{9 / 2} \\
\left({ }^{3} \mathrm{H}\right) 4 \mathrm{~d}^{2} \mathrm{H}_{11 / 2} \\
\left({ }^{3} \mathrm{H}\right) 4 \mathrm{~d}^{2} \mathrm{H}_{9 / 2} \\
\left({ }^{3} \mathrm{~F}\right) 4 \mathrm{~d}^{4} \mathrm{~F}_{9 / 2} \\
\left({ }^{3} \mathrm{~F}\right) 4 \mathrm{~d}^{2} \mathrm{G}_{9 / 2} \\
\left({ }^{3} \mathrm{G}\right) 4 \mathrm{~d}^{4} \mathrm{~F}_{9 / 2}\end{array}$ & $\begin{array}{l}4773.341 \\
4793.540 \\
5044.081 \\
5072.063 \\
5110.175 \\
5134.199 \\
5149.000 \\
5199.288 \\
5233.477 \\
5299.732 \\
5372.464 \\
5380.285 \\
5395.335 \\
5583.566 \\
5647.362 \\
6748.062\end{array}$ & $\begin{array}{l}-1.317 \\
-0.748 \\
-0.396 \\
-0.515 \\
-1.355 \\
+0.353 \\
-0.004 \\
-0.178 \\
-0.662 \\
-0.158 \\
-0.223 \\
-0.656 \\
+0.054 \\
-1.347 \\
-0.074 \\
-1.222\end{array}$ & $\begin{array}{l}4793.55 \\
5072.05 \\
5134.20 \\
5199.29 \\
5233.47 \\
5299.717 \\
5380.29 \\
5395.32\end{array}$ & $\begin{array}{l}\text { wrong, not observed } \\
\text { blend } \\
\text { blend } \\
\text { blend } \\
\text { computed too weak } \\
\text { lab } \\
\text { blend } \\
\text { computed too strong } \\
\text { blend } \\
\text { at the level of the noise }\end{array}$ \\
\hline 124636.116 & $\left({ }^{3} \mathrm{~F}\right) 4 \mathrm{f}$ & $3[5]$ & $9 / 2$ & $\begin{array}{l}103771.320 \\
104107.950 \\
104481.590 \\
104807.210 \\
104873.230 \\
104916.550\end{array}$ & $\begin{array}{l}\left({ }^{3} \mathrm{H}\right) 4 d^{4} \mathrm{G}_{9 / 2} \\
\left({ }^{3} \mathrm{P}\right) 4 \mathrm{~d}^{4} \mathrm{~F}_{7 / 2} \\
\left({ }^{3} \mathrm{H}\right) 4 \mathrm{~d}^{2} \mathrm{~F}_{7 / 2} \\
\left({ }^{3} \mathrm{H}\right) 4 \mathrm{~d}^{2} \mathrm{G}_{9 / 2} \\
\left({ }^{5} \mathrm{D}\right) 5 \mathrm{~d}^{4} \mathrm{D}_{7 / 2} \\
\left({ }^{3} \mathrm{H}\right) 4 \mathrm{~d}^{4} \mathrm{~F}_{9 / 2}\end{array}$ & $\begin{array}{l}4791.423 \\
4869.996 \\
4960.280 \\
5041.737 \\
5058.579 \\
5069.692 \\
\end{array}$ & $\begin{array}{l}-1.349 \\
-1.378 \\
-1.109 \\
-1.101 \\
-1.461 \\
-1.055\end{array}$ & 4960.28 & $\begin{array}{l}\text { at the level of the noise } \\
\text { blend } \\
\text { weak } \\
\text { weak } \\
\text { weak } \\
\text { weak }\end{array}$ \\
\hline
\end{tabular}


F. Castelli and R. L. Kurucz: New Fe II energy levels from stellar spectra

Table 8. continued.

\begin{tabular}{|c|c|c|c|c|c|c|c|c|c|}
\hline \multicolumn{4}{|c|}{ Upper level } & \multicolumn{2}{|c|}{ Lower level } & \multirow{2}{*}{$\begin{array}{c}\lambda(\mathrm{calc}) \\
\AA\end{array}$} & \multirow{2}{*}{$\begin{array}{c}\log g f \\
\text { KUR }\end{array}$} & \multirow{2}{*}{$\begin{array}{c}\lambda(\mathrm{obs}) \\
\AA\end{array}$} & \multirow[t]{2}{*}{ Notes } \\
\hline $\mathrm{cm}^{-1}$ & & & $J$ & $\mathrm{~cm}^{-1}$ & & & & & \\
\hline 124636.116 & cont. & & & $\begin{array}{l}104993.860 \\
105123.000 \\
105155.090 \\
105211.062 \\
105220.600 \\
105291.010 \\
105398.852 \\
105524.461 \\
105589.670 \\
105763.270 \\
105775.491 \\
106018.643 \\
106097.520 \\
106767.210 \\
106900.370 \\
106924.430 \\
109901.500 \\
110167.280\end{array}$ & $\begin{array}{l}\left({ }^{3} \mathrm{~F}\right) 4 d^{4} \mathrm{D}_{7 / 2} \\
\left({ }^{3} \mathrm{H}\right) 4 \mathrm{~d}^{2} \mathrm{G}_{7 / 2} \\
\left({ }^{3} \mathrm{~F}\right) 4 \mathrm{~d}^{4} \mathrm{G}_{9 / 2} \\
\left({ }^{5} \mathrm{D}\right) 5 \mathrm{~d}^{4} \mathrm{G}_{9 / 2} \\
\left({ }^{3} \mathrm{H}\right) 4 \mathrm{~d}^{4} \mathrm{~F}_{7 / 2} \\
\left({ }^{3} \mathrm{~F}\right) 4 \mathrm{~d}^{4} \mathrm{G}_{7 / 2} \\
\left({ }^{3} \mathrm{~F}\right) 4 \mathrm{~d}^{4} \mathrm{H}_{11 / 2} \\
\left({ }^{3} \mathrm{~F}\right) 4 \mathrm{~d}^{4} \mathrm{H}_{9 / 2} \\
\left({ }^{3} \mathrm{~F}\right) 4 \mathrm{~d}^{4} \mathrm{H}_{7 / 2} \\
\left({ }^{3} \mathrm{~F}\right) 4 \mathrm{~d}^{2} \mathrm{H}_{11 / 2} \\
\left({ }^{3} \mathrm{~F}\right) 4 \mathrm{~d}^{2} \mathrm{~F}_{7 / 2} \\
\left({ }^{3} \mathrm{~F}\right) 4 \mathrm{~d}^{2} \mathrm{H}_{9 / 2} \\
\left({ }^{3} \mathrm{H}\right) 4 \mathrm{~d}^{2} \mathrm{H}_{9 / 2} \\
\left({ }^{3} \mathrm{~F}\right) 4 \mathrm{~d}^{4} \mathrm{~F}_{7 / 2} \\
\left({ }^{3} \mathrm{~F}\right) 4 \mathrm{~d}^{2} \mathrm{G}_{7 / 2} \\
\left({ }^{3} \mathrm{~F}\right) 4 \mathrm{~d}^{2} \mathrm{G}_{9 / 2} \\
\left({ }^{3} \mathrm{G}\right) 4 \mathrm{~d}^{2} \mathrm{G}_{7 / 2} \\
\left({ }^{3} \mathrm{G}\right) 4 \mathrm{~d}^{4} \mathrm{~F}_{7 / 2}\end{array}$ & $\begin{array}{l}5089.646 \\
5123.331 \\
5131.770 \\
5146.557 \\
5149.085 \\
5167.827 . \\
5196.797 \\
5230.953 \\
5248.862 \\
5297.144 \\
5300.576 \\
5369.805 \\
5392.652 \\
5594.760 \\
5636.766 \\
5644.423 \\
6784.867 \\
6909.500\end{array}$ & $\begin{array}{l}-0.797 \\
-1.032 \\
-0.298 \\
-0.622 \\
+0.286 \\
-0.884 \\
-1.467 \\
-0.507 \\
-0.754 \\
-1.481 \\
-0.373 \\
-0.547 \\
-0.592 \\
-0.050 \\
-0.061 \\
-0.918 \\
-1.141 \\
-1.099\end{array}$ & $\begin{array}{l}5149.1 \\
5167.82 \\
5230.959 \\
5248.801 \\
\\
5369.81\end{array}$ & $\begin{array}{l}\text { weak } \\
\text { blend } \\
\text { blend } \\
\text { lab } \\
\text { computed too weak } \\
\text { at the level of the noise } \\
\text { computed too weak } \\
\text { blend } \\
\text { weak } \\
\text { weak } \\
\text { not obs, wrong } \\
\text { not obs, wrong } \\
\text { computed too weak } \\
\text { blend } \\
\text { at the level of the noise } \\
\text { at the level of the noise }\end{array}$ \\
\hline 124623.120 & $\left({ }^{3} \mathrm{~F}\right) 4 \mathrm{f}$ & $3[4]$ & $9 / 2$ & $\begin{array}{l}103921.630 \\
103983.510 \\
103986.330 \\
104916.550 \\
104993.860 \\
105123.000 \\
105155.090 \\
105211.062 \\
105220.600 \\
105291.010 \\
105398.852 \\
105449.540 \\
105524.461 \\
105763.270 \\
105775.491 \\
106018.640 \\
106097.520 \\
106900.370 \\
106924.430 \\
110570.300\end{array}$ & $\begin{array}{l}\left({ }^{3} \mathrm{H}\right) 4 \mathrm{~d}^{4} \mathrm{G}_{7 / 2} \\
\left({ }^{3} \mathrm{G}\right) 5 \mathrm{~s}^{2} \mathrm{G}_{7 / 2} \\
\left({ }^{3} \mathrm{H}\right) 4 \mathrm{~d}^{4} \mathrm{H}_{7 / 2} \\
\left({ }^{3} \mathrm{H}\right) 4 \mathrm{~d}^{4} \mathrm{~F}_{9 / 2} \\
\left({ }^{3} \mathrm{~F}\right) 4 \mathrm{~d}^{4} \mathrm{D}_{7 / 2} \\
\left({ }^{3} \mathrm{H}\right) 4 \mathrm{~d}^{2} \mathrm{G}_{7 / 2} \\
\left({ }^{3} \mathrm{~F}\right) 4 \mathrm{~d}^{4} \mathrm{G}_{9 / 2} \\
\left({ }^{5} \mathrm{D}\right) 5 \mathrm{~d}^{4} \mathrm{G}_{9 / 2} \\
\left({ }^{3} \mathrm{H}\right) 4 \mathrm{~d}^{4} \mathrm{~F}_{7 / 2} \\
\left({ }^{3} \mathrm{~F}\right) 4 \mathrm{~d}^{4} \mathrm{G}_{7 / 2} \\
\left({ }^{3} \mathrm{~F}\right) 4 \mathrm{~d}^{4} \mathrm{H}_{11 / 2} \\
\left({ }^{5} \mathrm{D}\right) 5 \mathrm{~d}^{4} \mathrm{G}_{7 / 2} \\
\left({ }^{3} \mathrm{~F}\right) 4 \mathrm{~d}^{4} \mathrm{H}_{9 / 2} \\
\left({ }^{3} \mathrm{~F}\right) 4 \mathrm{~d}^{2} \mathrm{H}_{11 / 2} \\
\left({ }^{3} \mathrm{~F}\right) 4 \mathrm{~d}^{2} \mathrm{~F}_{7 / 2} \\
\left({ }^{3} \mathrm{~F}\right) 4 \mathrm{~d}^{2} \mathrm{H}_{9 / 2} \\
\left({ }^{3} \mathrm{H}\right) 4 \mathrm{~d}^{2} \mathrm{H}_{9 / 2} \\
\left({ }^{3} \mathrm{~F}\right) 4 \mathrm{~d}^{2} \mathrm{G}_{7 / 2} \\
\left({ }^{3} \mathrm{~F}\right) 4 \mathrm{~d}^{2} \mathrm{G}_{9 / 2} \\
\left({ }^{3} \mathrm{G}\right) 4 \mathrm{~d}^{2} \mathrm{~F}_{7 / 2}\end{array}$ & $\begin{array}{l}4829.221 \\
4843.700 \\
4844.361 \\
5073.036 \\
5093.016 \\
5126.745 \\
5135.196 \\
5150.003 \\
5152.534 \\
5171.301 \\
5200.310 \\
5214.058 \\
5234.513 \\
5300.794 \\
5304.231 \\
5373.555 \\
5396.435 \\
5640.900 \\
5648.568 \\
7114.048\end{array}$ & $\begin{array}{l}-1.017 \\
-1.308 \\
-1.133 \\
-1.028 \\
-1.142 \\
-0.382 \\
-0.318 \\
-0.755 \\
-1.333 \\
+0.425 \\
-1.359 \\
-0.628 \\
-0.157 \\
-1.386 \\
-0.076 \\
-1.277 \\
-0.899 \\
-0.389 \\
-0.369 \\
-1.243\end{array}$ & $\begin{array}{l}5093.01 \\
5126.75 \\
5150.02 \\
5171.305\end{array}$ & $\begin{array}{l}\text { computed too weak } \\
\text { computed too strong, not obs } \\
\text { weak } \\
\text { lab, blend } \\
\text { blend } \\
\text { blend } \\
\text { blend } \\
\text { blend } \\
\text { blend } \\
\text { blend } \\
\text { blend } \\
\text { computed too weak } \\
\text { computed too strong } \\
\text { blend }\end{array}$ \\
\hline 124620.914 & $\left({ }^{3} \mathrm{~F}\right) 4 \mathrm{f}$ & $3[4]$ & $7 / 2$ & $\begin{array}{l}103921.630 \\
104023.910 \\
104569.230 \\
104993.860 \\
105123.000 \\
105155.090 \\
105234.237 \\
105291.010 \\
105379.430 \\
105414.180 \\
105449.540 \\
105524.461 \\
105711.730 \\
105775.491 \\
106208.560 \\
106866.760 \\
106900.370 \\
106924.430 \\
107407.800\end{array}$ & $\begin{array}{l}\left({ }^{3} \mathrm{H}\right) 4 d^{4} \mathrm{G}_{7 / 2} \\
\left({ }^{3} \mathrm{H}\right) 4 \mathrm{~d}^{4} \mathrm{G}_{5 / 2} \\
\left({ }^{3} \mathrm{P}\right) 4 \mathrm{~d}^{4} \mathrm{~F}_{5 / 2} \\
\left({ }^{3} \mathrm{~F}\right) 4 \mathrm{~d}^{4} \mathrm{D}_{7 / 2} \\
\left({ }^{3} \mathrm{H}\right) 4 \mathrm{~d}^{2} \mathrm{G}_{5 / 2} \\
\left({ }^{3} \mathrm{~F}\right) 4 \mathrm{~d}^{4} \mathrm{G}_{9 / 2} \\
\left({ }^{3} \mathrm{H}\right) 4 \mathrm{~d}^{4} \mathrm{~F}_{5 / 2} \\
\left({ }^{3} \mathrm{~F}\right) 4 \mathrm{~d}^{4} \mathrm{G}_{7 / 2} \\
\left.{ }^{3} \mathrm{~F}\right) 4 \mathrm{~d}^{4} \mathrm{D}_{5 / 2} \\
\left({ }^{3} \mathrm{~F}\right) 4 \mathrm{~d}^{4} \mathrm{G}_{5 / 2} \\
\left({ }^{5} \mathrm{D}\right) 5 \mathrm{~d}^{4} \mathrm{G}_{7 / 2} \\
\left({ }^{3} \mathrm{~F}\right) 4 \mathrm{~d}^{4} \mathrm{H}_{9 / 2} \\
\left({ }^{5} \mathrm{D}\right) 5 \mathrm{~d}^{6} \mathrm{~S}_{5 / 2} \\
\left({ }^{3} \mathrm{~F}\right) 4 \mathrm{~d}^{2} \mathrm{~F}_{7 / 2} \\
\left({ }^{3} \mathrm{~F}\right) 4 \mathrm{~d}^{2} \mathrm{~F}_{5 / 2} \\
\left({ }^{3} \mathrm{~F}\right) 4 \mathrm{~d}^{4} \mathrm{~F}_{5 / 2} \\
\left({ }^{3} \mathrm{~F}\right) 4 \mathrm{~d}^{2} \mathrm{G}_{7 / 2} \\
\left({ }^{3} \mathrm{~F}\right) 4 \mathrm{~d}^{2} \mathrm{G}_{9 / 2} \\
\left({ }^{3} \mathrm{~F}\right) 4 \mathrm{~d}^{2} \mathrm{D}_{5 / 2}\end{array}$ & $\begin{array}{l}4829.735 \\
4853.719 \\
4985.721 \\
5093.588 \\
5127.325 \\
5135.778 \\
5156.745 \\
5171.891 \\
5195.658 \\
5205.058 \\
5214.658 \\
5235.117 \\
5286.964 \\
5304.852 \\
5429.627 \\
5630.922 \\
5641.602 \\
5649.272 \\
5807.914\end{array}$ & $\begin{array}{l}-1.435 \\
-0.883 \\
-0.873 \\
-1.437 \\
-0.784 \\
-1.386 \\
-0.254 \\
+0.011 \\
-0.478 \\
-0.783 \\
-1.042 \\
-1.185 \\
-0.934 \\
-0.525 \\
-0.531 \\
-1.421 \\
-0.724 \\
-1.404 \\
-0.295\end{array}$ & $\begin{array}{c}5304.87 \\
5429.62 \\
5641.61 \\
5807.9 \\
\end{array}$ & $\begin{array}{l}\text { weak } \\
\text { blend } \\
\text { blend } \\
\text { weak } \\
\text { blend } \\
\text { lab } \\
\text { blend } \\
\text { weak } \\
\text { blend } \\
\text { blend } \\
\text { blend } \\
\text { computed too weak } \\
\text { weak } \\
\text { weak } \\
\text { not observed } \\
\text { blend }\end{array}$ \\
\hline
\end{tabular}


Table 8. continued.

\begin{tabular}{|c|c|c|c|c|c|c|c|c|c|}
\hline \multicolumn{4}{|c|}{ Upper level } & \multicolumn{2}{|c|}{ Lower level } & \multirow{2}{*}{$\begin{array}{c}\lambda(\text { calc }) \\
\AA\end{array}$} & \multirow{2}{*}{$\begin{array}{c}\log g f \\
\text { KUR }\end{array}$} & \multirow{2}{*}{$\begin{array}{c}\lambda(\mathrm{obs}) \\
\AA\end{array}$} & \multirow[t]{2}{*}{ Notes } \\
\hline $\mathrm{cm}^{-1}$ & & & $J$ & $\mathrm{~cm}^{-1}$ & & & & & \\
\hline 124641.989 & $\left({ }^{3} \mathrm{~F}\right) 4 \mathrm{f}$ & $3[3]$ & $7 / 2$ & $\begin{array}{l}104107.950 \\
104120.270 \\
104481.590 \\
105123.000 \\
105155.090 \\
105211.062 \\
105220.600 \\
105291.010 \\
105379.430 \\
105414.180 \\
105589.670 \\
105711.730 \\
105775.491 \\
106097.520 \\
106208.560 \\
106767.210 \\
106796.660 \\
106866.760 \\
106900.370 \\
106924.430 \\
107407.800 \\
110167.280 \\
110611.800\end{array}$ & $\begin{array}{l}\left({ }^{3} \mathrm{P}\right) 4 \mathrm{~d}^{4} \mathrm{~F}_{7 / 2} \\
\left({ }^{5} \mathrm{D}\right) 5 \mathrm{~d}^{6} \mathrm{P}_{5 / 2} \\
\left({ }^{3} \mathrm{H}\right) 4 \mathrm{~d}^{2} \mathrm{~F}_{7 / 2} \\
\left({ }^{3} \mathrm{H}\right) 4 \mathrm{~d}^{2} \mathrm{G}_{7 / 2} \\
\left({ }^{3} \mathrm{~F}\right) 4 \mathrm{~d}^{4} \mathrm{G}_{9 / 2} \\
\left({ }^{5} \mathrm{D}\right) 5 \mathrm{~d}^{4} \mathrm{G}_{9 / 2} \\
\left({ }^{3} \mathrm{H}\right) 4 \mathrm{~d}^{4} \mathrm{~F}_{7 / 2} \\
\left({ }^{3} \mathrm{~F}\right) 4 \mathrm{~d}^{4} \mathrm{G}_{7 / 2} \\
\left({ }^{3} \mathrm{~F}\right) 4 \mathrm{~d}^{4} \mathrm{D}_{5 / 2} \\
\left({ }^{3} \mathrm{~F}\right) 4 \mathrm{~d}^{4} \mathrm{G}_{5 / 2} \\
\left({ }^{3} \mathrm{~F}\right) 4 \mathrm{~d}^{4} \mathrm{H}_{7 / 2} \\
\left({ }^{5} \mathrm{D}\right) 5 \mathrm{~d}^{6} \mathrm{~S}_{5 / 2} \\
\left({ }^{3} \mathrm{~F}\right) 4 \mathrm{~d}^{2} \mathrm{~F}_{7 / 2} \\
\left({ }^{3} \mathrm{H}\right) 4 \mathrm{~d}^{2} \mathrm{H}_{9 / 2} \\
\left({ }^{3} \mathrm{~F}\right) 4 \mathrm{~d}^{2} \mathrm{~F}_{5 / 2} \\
\left({ }^{3} \mathrm{~F}\right) 4 \mathrm{~d}^{4} \mathrm{~F}_{7 / 2} \\
\left({ }^{3} \mathrm{~F}\right) 4 \mathrm{~d}^{4} \mathrm{P}_{5 / 2} \\
\left({ }^{3} \mathrm{~F}\right) 4 \mathrm{~d}^{4} \mathrm{~F}_{5 / 2} \\
\left({ }^{3} \mathrm{~F}\right) 4 \mathrm{~d}^{2} \mathrm{G}_{7 / 2} \\
\left({ }^{3} \mathrm{~F}\right) 4 \mathrm{~d}^{2} \mathrm{G}_{9 / 2} \\
\left({ }^{3} \mathrm{~F}\right) 4 \mathrm{~d}^{2} \mathrm{D}_{5 / 2} \\
\left({ }^{3} \mathrm{G}\right) 4 \mathrm{~d}^{4} \mathrm{~F}_{7 / 2} \\
\left({ }^{3} \mathrm{G}\right) 4 \mathrm{~d}^{2} \mathrm{~F}_{5 / 2}\end{array}$ & $\begin{array}{l}4868.603 \\
4871.525 \\
4958.835 \\
5121.789 \\
5130.223 \\
5145.002 \\
5147.528 \\
5166.258 \\
5189.973 \\
5199.353 \\
5247.244 \\
5281.078 \\
5298.926 \\
5390.945 \\
5423.419 \\
5592.922 \\
5602.152 \\
5624.245 \\
5634.900 \\
5642.552 \\
5800.811 \\
6906.696 \\
7125.523\end{array}$ & $\begin{array}{l}-1.393 \\
-1.423 \\
-1.370 \\
-0.828 \\
-0.928 \\
-1.290 \\
-0.014 \\
-1.096 \\
-0.210 \\
-1.041 \\
-0.996 \\
-0.874 \\
-0.405 \\
-1.384 \\
-0.138 \\
-0.422 \\
-0.795 \\
-1.195 \\
-0.588 \\
-1.377 \\
-0.993 \\
-1.294 \\
-1.233\end{array}$ & $\begin{array}{l}5147.52 \\
5247.25\end{array}$ & $\begin{array}{l}\text { blend } \\
\text { blend } \\
\text { at the level of the noise } \\
\text { weak } \\
\text { blend } \\
\text { blend } \\
\text { weak } \\
\text { not observed } \\
\text { blend } \\
\text { blend } \\
\text { lab } \\
\text { wrong } \\
\text { blend } \\
\text { blend } \\
\text { computed too weak } \\
\text { at the level of the noise } \\
\text { at the level of the noise } \\
\text { at the level of the noise } \\
\text { at the level of the noise }\end{array}$ \\
\hline 124653.022 & $\left({ }^{3} \mathrm{~F}\right) 4 \mathrm{f}$ & $3[3]$ & $5 / 2$ & $\begin{array}{l}104023.910 \\
104569.230 \\
104839.998 \\
105123.000 \\
105234.237 \\
105291.010 \\
105317.440 \\
105379.430 \\
105414.180 \\
105460.230 \\
105711.730 \\
105775.491 \\
106208.560 \\
106846.650 \\
107065.930 \\
107407.800 \\
107430.250 \\
108105.900\end{array}$ & $\begin{array}{l}\left({ }^{3} \mathrm{H}\right) 4 \mathrm{~d}^{4} \mathrm{G}_{5 / 2} \\
\left({ }^{3} \mathrm{P}\right) 4 \mathrm{~d}^{4} \mathrm{~F}_{5 / 2} \\
\left({ }^{3} \mathrm{P}\right) 4 \mathrm{~d}^{2} \mathrm{D}_{3 / 2} \\
\left({ }^{3} \mathrm{H}\right) 4 \mathrm{~d}^{2} \mathrm{G}_{7 / 2} \\
\left({ }^{3} \mathrm{H}\right) 4 \mathrm{~d}^{4} \mathrm{~F}_{5 / 2} \\
\left({ }^{3} \mathrm{~F}\right) 4 \mathrm{~d}^{4} \mathrm{G}_{7 / 2} \\
\left({ }^{3} \mathrm{P}\right) 4 \mathrm{~d}^{2} \mathrm{P}_{3 / 2} \\
\left({ }^{3} \mathrm{~F}\right) 4 \mathrm{~d}^{4} \mathrm{D}_{5 / 2} \\
\left({ }^{3} \mathrm{~F}\right) 4 \mathrm{~d}^{4} \mathrm{G}_{5 / 2} \\
\left({ }^{3} \mathrm{~F}\right) 4 \mathrm{~d}^{4} \mathrm{D}_{3 / 2} \\
\left({ }^{5} \mathrm{D}\right) 5 \mathrm{~d}^{6} \mathrm{~S}_{5 / 2} \\
\left({ }^{3} \mathrm{~F}\right) 4 \mathrm{~d}^{2} \mathrm{~F}_{7 / 2} \\
\left({ }^{3} \mathrm{~F}\right) 4 \mathrm{~d}^{2} \mathrm{~F}_{5 / 2} \\
\left({ }^{3} \mathrm{~F}\right) 4 \mathrm{~d}^{4} \mathrm{~F}_{3 / 2} \\
\left({ }^{3} \mathrm{~F}\right) 4 \mathrm{~d}^{4} \mathrm{P}_{3 / 2} \\
\left({ }^{3} \mathrm{~F}\right) 4 \mathrm{~d}^{2} \mathrm{D}_{5 / 2} \\
\left({ }^{3} \mathrm{~F}\right) 4 \mathrm{~d}^{2} \mathrm{D}_{3 / 2} \\
\left({ }^{3} \mathrm{~F}\right) 4 \mathrm{~d}^{2} \mathrm{P}_{3 / 2}\end{array}$ & $\begin{array}{l}4846.164 \\
4977.751 \\
5045.778 \\
5118.896 \\
5148.219 \\
5163.314 \\
5170.372 \\
5187.002 \\
5196.371 \\
5208.839 \\
5278.002 \\
5295.829 \\
5420.175 \\
5614.409 \\
5684.411 \\
5797.100 \\
5804.657 \\
6041.674\end{array}$ & $\begin{array}{l}-1.115 \\
-0.819 \\
-0.981 \\
-1.484 \\
-0.286 \\
-0.700 \\
-1.129 \\
-0.628 \\
-0.956 \\
-0.132 \\
-1.442 \\
-1.021 \\
-0.824 \\
-0.773 \\
-1.018 \\
-0.273 \\
-0.981 \\
-0.519\end{array}$ & $\begin{array}{l}4977.75 \\
5045.79 \\
\\
5163.29 \\
5187.0 \\
5208.862\end{array}$ & $\begin{array}{l}\text { weak } \\
\text { computed too weak } \\
\text { computed too weak } \\
\text { computed too strong } \\
\text { weak } \\
\text { blend } \\
\text { lab, computed too strong } \\
\text { blend } \\
\text { computed too weak } \\
\text { computed too strong } \\
\text { at the level of the noise }\end{array}$ \\
\hline 124731.762 & $\left({ }^{3} \mathrm{~F}\right) 4 \mathrm{f}$ & $3[0]$ & $1 / 2$ & $\begin{array}{l}104189.380 \\
104588.710 \\
104736.460 \\
105460.230 \\
105477.920 \\
105518.140 \\
107065.930 \\
107176.100 \\
107430.250 \\
108105.900 \\
\end{array}$ & $\begin{array}{l}\left({ }^{5} \mathrm{D}\right) 5 \mathrm{~d}^{4} \mathrm{P}_{3 / 2} \\
\left({ }^{5} \mathrm{D}\right) 5 \mathrm{~d}^{6} \mathrm{D}_{3 / 2} \\
\left({ }^{3} \mathrm{P}\right) 4 \mathrm{~d}^{2} \mathrm{P}_{1 / 2} \\
\left({ }^{3} \mathrm{~F}\right) 4 \mathrm{~d}^{4} \mathrm{D}_{3 / 2} \\
\left({ }^{3} \mathrm{~F}\right) 4 \mathrm{~d}^{4} \mathrm{D}_{1 / 2} \\
\left({ }^{3} \mathrm{H}\right) 4 \mathrm{~d}^{4} \mathrm{~F}_{3 / 2} \\
\left({ }^{3} \mathrm{~F}\right) 4 \mathrm{~d}^{4} \mathrm{P}_{3 / 2} \\
\left({ }^{5} \mathrm{D}\right) 5 \mathrm{~d}^{4} \mathrm{P}_{1 / 2} \\
\left({ }^{3} \mathrm{~F}\right) 4 \mathrm{~d}^{2} \mathrm{D}_{3 / 2} \\
\left({ }^{3} \mathrm{~F}\right) 4 \mathrm{~d}^{2} \mathrm{P}_{3 / 2}\end{array}$ & $\begin{array}{l}4866.625 \\
4963.106 \\
4999.780 \\
5187.556 \\
5192.323 \\
5203.192 \\
5659.074 \\
5694.588 \\
5778.239 \\
6013.060 \\
\end{array}$ & $\begin{array}{l}-0.710 \\
-1.473 \\
-1.476 \\
-1.137 \\
-0.902 \\
-0.854 \\
-0.650 \\
-0.810 \\
-0.939 \\
-1.184 \\
\end{array}$ & $\begin{array}{l}5659.05 \\
5694.59\end{array}$ & $\begin{array}{l}\text { on the } \mathrm{H}_{\beta} \text { wing } \\
\text { blend } \\
\text { blend } \\
\text { computed too weak } \\
\text { good agreement } \\
\text { blend }\end{array}$ \\
\hline
\end{tabular}


F. Castelli and R. L. Kurucz: New Fe II energy levels from stellar spectra

Table 8. continued.

\begin{tabular}{|c|c|c|c|c|c|c|c|c|c|}
\hline \multicolumn{4}{|c|}{ Upper level } & \multicolumn{2}{|c|}{ Lower level } & \multirow{2}{*}{$\begin{array}{c}\lambda(\text { calc }) \\
\AA\end{array}$} & \multirow{2}{*}{$\begin{array}{c}\log g f \\
\text { KUR }\end{array}$} & \multirow{2}{*}{$\begin{array}{c}\lambda(\mathrm{obs}) \\
\AA\end{array}$} & \multirow[t]{2}{*}{ Notes } \\
\hline $\mathrm{cm}^{-1}$ & & & $J$ & $\mathrm{~cm}^{-1}$ & & & & & \\
\hline 124803.873 & $\left({ }^{3} \mathrm{~F}\right) 4 \mathrm{f}$ & $2[5]$ & $11 / 2$ & $\begin{array}{l}103771.320 \\
104807.210 \\
105524.461 \\
106018.643 \\
106097.520 \\
106924.430 \\
109625.200\end{array}$ & $\begin{array}{l}\left({ }^{3} \mathrm{H}\right) 4 \mathrm{~d}^{4} \mathrm{G}_{9 / 2} \\
\left({ }^{3} \mathrm{H}\right) 4 \mathrm{~d}^{2} \mathrm{G}_{9 / 2} \\
\left({ }^{3} \mathrm{~F}\right) 4 \mathrm{~d}^{4} \mathrm{H}_{9 / 2} \\
\left({ }^{3} \mathrm{~F}\right) 4 \mathrm{~d}^{2} \mathrm{H}_{9 / 2} \\
\left({ }^{3} \mathrm{H}\right) 4 \mathrm{~d}^{2} \mathrm{H}_{9 / 2} \\
\left({ }^{3} \mathrm{~F}\right) 4 \mathrm{~d}^{2} \mathrm{G}_{9 / 2} \\
\left({ }^{3} \mathrm{G}\right) 4 \mathrm{~d}^{2} \mathrm{G}_{9 / 2}\end{array}$ & $\begin{array}{l}4753.206 \\
4999.441 \\
5185.437 \\
5321.852 \\
5344.292 \\
5591.464 \\
6586.373\end{array}$ & $\begin{array}{l}-1.359 \\
-1.315 \\
+0.377 \\
+0.731 \\
-1.008 \\
-0.173 \\
-1.344\end{array}$ & $\begin{array}{l}5185.422 \\
5321.83 \\
5344.28\end{array}$ & $\begin{array}{l}\text { lab } \\
\text { lab } \\
\text { computed too strong } \\
\text { not observed }\end{array}$ \\
\hline 124809.727 & $\left({ }^{3} \mathrm{~F}\right) 4 \mathrm{f}$ & $2[5]$ & $9 / 2$ & $\begin{array}{l}103921.630 \\
103983.510 \\
105291.010 \\
105449.540 \\
105524.461 \\
105589.670 \\
105775.491 \\
106018.643 \\
106767.210 \\
106900.370\end{array}$ & $\begin{array}{l}\left({ }^{3} \mathrm{H}\right) 4 d^{4} \mathrm{G}_{7 / 2} \\
\left({ }^{3} \mathrm{G}\right) 5 \mathrm{~s}^{2} \mathrm{G}_{7 / 2} \\
\left({ }^{3} \mathrm{~F}\right) 4 \mathrm{~d}^{4} \mathrm{G}_{7 / 2} \\
\left({ }^{5} \mathrm{D}\right) 5 \mathrm{~d}^{4} \mathrm{G}_{7 / 2} \\
\left({ }^{3} \mathrm{~F}\right) 4 \mathrm{~d}^{4} \mathrm{H}_{9 / 2} \\
\left({ }^{3} \mathrm{~F}\right) 4 \mathrm{~d}^{4} \mathrm{H}_{7 / 2} \\
\left({ }^{3} \mathrm{~F}\right) 4 \mathrm{~d}^{2} \mathrm{~F}_{7 / 2} \\
\left({ }^{3} \mathrm{~F}\right) 4 \mathrm{~d}^{2} \mathrm{H}_{9 / 2} \\
\left({ }^{3} \mathrm{~F}\right) 4 \mathrm{~d}^{4} \mathrm{~F}_{7 / 2} \\
\left({ }^{3} \mathrm{~F}\right) 4 \mathrm{~d}^{2} \mathrm{G}_{7 / 2}\end{array}$ & $\begin{array}{l}4786.078 \\
4800.298 \\
5121.860 \\
5163.801 \\
5183.862 \\
5201.450 \\
5252.229 \\
5320.193 \\
5540.925 \\
5582.123\end{array}$ & $\begin{array}{l}-1.434 \\
-1.342 \\
-1.107 \\
-1.335 \\
-1.227 \\
+0.802 \\
-1.121 \\
-0.866 \\
-1.367 \\
-0.405\end{array}$ & 5582.12 & $\begin{array}{l}\text { blend } \\
\text { blend } \\
\text { blend } \\
\text { lab } \\
\text { blend }\end{array}$ \\
\hline 124793.905 & $\left({ }^{3} \mathrm{~F}\right) 4 \mathrm{f}$ & $2[4]$ & $9 / 2$ & $\begin{array}{l}103921.630 \\
103986.330 \\
104481.590 \\
105123.000 \\
105220.600 \\
105291.010 \\
105449.540 \\
105524.460 \\
105589.670 \\
105775.491 \\
106018.640 \\
106900.370 \\
106924.430 \\
109901.500 \\
110167.280 \\
110570.300\end{array}$ & $\begin{array}{l}\left({ }^{3} \mathrm{H}\right) 4 d^{4} \mathrm{G}_{7 / 2} \\
\left({ }^{3} \mathrm{H}\right) 4 \mathrm{~d}^{4} \mathrm{H}_{7 / 2} \\
\left({ }^{3} \mathrm{H}\right) 4 \mathrm{~d}^{2} \mathrm{~F}_{7 / 2} \\
\left({ }^{3} \mathrm{H}\right) 4 \mathrm{~d}^{2} \mathrm{G}_{7 / 2} \\
\left({ }^{3} \mathrm{H}\right) 4 \mathrm{~d}^{4} \mathrm{~F}_{7 / 2} \\
\left({ }^{3} \mathrm{~F}\right) 4 \mathrm{~d}^{4} \mathrm{G}_{7 / 2} \\
\left({ }^{5} \mathrm{D}\right) 5 \mathrm{~d}^{4} \mathrm{G}_{7 / 2} \\
\left({ }^{3} \mathrm{~F}\right) 4 \mathrm{~d}^{4} \mathrm{H}_{9 / 2} \\
\left({ }^{3} \mathrm{~F}\right) 4 \mathrm{~d}^{4} \mathrm{H}_{7 / 2} \\
\left({ }^{3} \mathrm{~F}\right) 4 \mathrm{~d}^{2} \mathrm{~F}_{7 / 2} \\
\left({ }^{3} \mathrm{~F}\right) 4 \mathrm{~d}^{2} \mathrm{H}_{9 / 2} \\
\left({ }^{3} \mathrm{~F}\right) 4 \mathrm{~d}^{2} \mathrm{G}_{7 / 2} \\
\left({ }^{3} \mathrm{~F}\right) 4 \mathrm{~d}^{2} \mathrm{G}_{9 / 2} \\
\left({ }^{3} \mathrm{G}\right) 4 \mathrm{~d}^{2} \mathrm{G}_{7 / 2} \\
\left({ }^{3} \mathrm{G}\right) 4 \mathrm{~d}^{4} \mathrm{~F}_{7 / 2} \\
\left({ }^{3} \mathrm{G}\right) 4 \mathrm{~d}^{2} \mathrm{~F}_{7 / 2}\end{array}$ & $\begin{array}{l}4789.706 \\
4804.599 \\
4921.748 \\
5082.234 \\
5107.576 \\
5126.016 \\
5168.025 \\
5188.118 \\
5205.735 \\
5256.599 \\
5324.675 \\
5587.059 \\
5594.582 \\
6712.979 \\
6834.961 \\
7028.628\end{array}$ & $\begin{array}{l}-1.174 \\
-1.426 \\
-1.081 \\
-0.341 \\
-0.574 \\
+0.065 \\
-1.175 \\
-0.544 \\
-0.340 \\
-0.442 \\
-0.131 \\
+0.466 \\
-1.114 \\
-1.436 \\
-1.262 \\
-1.389\end{array}$ & $\begin{array}{l}5126.00 \\
5188.12 \\
5256.599 \\
5234.68\end{array}$ & $\begin{array}{l}\text { computed too weak } \\
\text { blend } \\
\text { blend } \\
\text { blend } \\
\text { blend } \\
\text { lab. } \\
\text { good agreement } \\
\text { good agreement } \\
\text { blend } \\
\text { good agreement } \\
\text { good agreementy= } \\
\text { blend } \\
\text { blend }\end{array}$ \\
\hline 124783.748 & $\left({ }^{3} \mathrm{~F}\right) 4 \mathrm{f}$ & $2[4]$ & $7 / 2$ & $\begin{array}{l}104023.910 \\
104120.270 \\
104209.610 \\
104569.230 \\
105123.000 \\
105291.010 \\
105414.180 \\
105589.670 \\
105630.750 \\
106018.640 \\
106208.560 \\
106900.370 \\
107407.800 \\
110611.800\end{array}$ & $\begin{array}{l}\left({ }^{3} \mathrm{H}\right) 4 d^{4} \mathrm{G}_{5 / 2} \\
\left({ }^{5} \mathrm{D}\right) 5 \mathrm{~d}^{6} \mathrm{P}_{5 / 2} \\
\left({ }^{3} \mathrm{H}\right) 4 \mathrm{~d}^{2} \mathrm{~F}_{5 / 2} \\
\left({ }^{3} \mathrm{P}\right) 4 \mathrm{~d}^{4} \mathrm{~F}_{5 / 2} \\
\left({ }^{3} \mathrm{H}\right) 4 \mathrm{~d}^{2} \mathrm{G}_{7 / 2} \\
\left({ }^{3} \mathrm{~F}\right) 4 \mathrm{~d}^{4} \mathrm{G}_{7 / 2} \\
\left({ }^{3} \mathrm{~F}\right) 4 \mathrm{~d}^{4} \mathrm{G}_{5 / 2} \\
\left({ }^{3} \mathrm{~F}\right) 4 \mathrm{~d}^{4} \mathrm{H}_{7 / 2} \\
\left({ }^{5} \mathrm{D}\right) 5 \mathrm{~d}^{4} \mathrm{G}_{5 / 2} \\
\left({ }^{3} \mathrm{~F}\right) 4 \mathrm{~d}^{2} \mathrm{H}_{9 / 2} \\
\left({ }^{3} \mathrm{~F}\right) 4 \mathrm{~d}^{2} \mathrm{~F}_{5 / 2} \\
\left({ }^{3} \mathrm{~F}\right) 4 \mathrm{~d}^{2} \mathrm{G}_{7 / 2} \\
\left({ }^{3} \mathrm{~F}\right) 4 \mathrm{~d}^{2} \mathrm{D}_{5 / 2} \\
\left({ }^{3} \mathrm{G}\right) 4 \mathrm{~d}^{2} \mathrm{~F}_{5 / 2}\end{array}$ & $\begin{array}{l}4815.647 \\
4838.105 \\
4859.114 \\
4945.559 \\
5084.859 \\
5128.687 \\
5161.300 \\
5208.490 \\
5219.661 \\
5327.557 \\
5382.029 \\
5590.233 \\
5753.486 \\
7054.248\end{array}$ & $\begin{array}{l}-0.780 \\
-1.439 \\
-1.499 \\
-1.176 \\
-1.401 \\
-0.876 \\
+0.512 \\
-0.196 \\
-0.923 \\
-1.482 \\
-0.281 \\
-0.326 \\
-0.930 \\
-1.377\end{array}$ & $\begin{array}{l}5161.3 \\
5208.501\end{array}$ & $\begin{array}{l}\text { not observed } \\
\text { weak } \\
\text { blend } \\
\text { lab, computed too strong } \\
\text { blend }\end{array}$ \\
\hline
\end{tabular}


A\&A 520, A57 (2010)

Table 9. Fe II lines in the $3800-8000 \AA$ A region with $\log g f \geq-1.5$ and $3 \mathrm{~d}^{6}\left({ }^{3} \mathrm{G}\right) 4 \mathrm{f}$ energy levels as upper levels.

\begin{tabular}{|c|c|c|c|c|c|c|c|c|c|}
\hline $\mathrm{cm}^{-1}$ & Jpper leve & & $J$ & $\begin{array}{l}\text { Low } \\
\mathrm{cm}^{-1}\end{array}$ & r level & $\begin{array}{c}\lambda(\text { calc }) \\
\AA\end{array}$ & $\begin{array}{c}\log g f \\
\text { KUR }\end{array}$ & $\begin{array}{c}\lambda(\mathrm{obs}) \\
\AA \\
\end{array}$ & Notes \\
\hline 127507.241 & $\left({ }^{3} G\right) 4 f$ & $5[8]$ & $17 / 2$ & $\begin{array}{l}103878.370 \\
108337.860\end{array}$ & $\begin{array}{l}\left({ }^{3} \mathrm{H}\right) 4 d^{4} I_{15 / 2} \\
\left({ }^{3} \mathrm{G}\right) 4 d^{4} I_{15 / 2}\end{array}$ & $\begin{array}{l}4230.919 \\
5215.200\end{array}$ & $\begin{array}{l}-1.017 \\
+1.119\end{array}$ & $\begin{array}{l}4230.93 \\
5215.21\end{array}$ & \\
\hline 127524.122 & $\left({ }^{3} \mathrm{G}\right) 4 \mathrm{f}$ & $5[8]$ & $15 / 2$ & $\begin{array}{l}104064.670 \\
104622.300 \\
108133.440 \\
108463.910 \\
108648.695 \\
109049.600\end{array}$ & $\begin{array}{l}\left({ }^{3} \mathrm{H}\right) 4 \mathrm{~d}^{4} \mathrm{I}_{13 / 2} \\
\left({ }^{3} \mathrm{H}\right) 4 \mathrm{~d}^{2} \mathrm{I}_{13 / 2} \\
\left({ }^{3} \mathrm{G}\right) 4 \mathrm{~d}^{4} \mathrm{H}_{13 / 2} \\
\left({ }^{3} \mathrm{G}\right) 4 \mathrm{~d}^{4} \mathrm{I}_{13 / 2} \\
\left({ }^{1} \mathrm{I}\right) 5 \mathrm{~s} \mathrm{e}{ }^{2} \mathrm{I}_{13 / 2} \\
\left({ }^{3} \mathrm{G}\right) 4 \mathrm{~d}^{2} \mathrm{I}_{13 / 2}\end{array}$ & $\begin{array}{l}4261.475 \\
4365.238 \\
5155.680 \\
5245.071 \\
5296.420 \\
5411.356\end{array}$ & $\begin{array}{l}-1.477 \\
-1.210 \\
-0.971 \\
+0.889 \\
-0.047 \\
+0.449\end{array}$ & $\begin{array}{l}5245.073 \\
5296.418\end{array}$ & $\begin{array}{l}\text { lab, J78 } \\
\text { blend }\end{array}$ \\
\hline 127484.653 & $\left({ }^{3} \mathrm{G}\right) 4 \mathrm{f}$ & $5[7]$ & $15 / 2$ & $\begin{array}{l}108133.440 \\
108337.860 \\
108463.910 \\
108648.695 \\
109049.600\end{array}$ & $\begin{array}{l}\left({ }^{3} \mathrm{G}\right) 4 \mathrm{~d}^{4} \mathrm{H}_{13 / 2} \\
\left({ }^{3} \mathrm{G}\right) 4 \mathrm{~d}^{4} \mathrm{I}_{15 / 2} \\
\left({ }^{3} \mathrm{G}\right) 4 \mathrm{~d}^{4} \mathrm{I}_{13 / 2} \\
\left({ }^{1} \mathrm{I}\right) 5 \mathrm{~s} \mathrm{e} \mathrm{e}^{2} \mathrm{I}_{13 / 2} \\
\left({ }^{3} \mathrm{G}\right) 4 \mathrm{~d}^{2} \mathrm{I}_{13 / 2}\end{array}$ & $\begin{array}{l}5166.196 \\
5221.353 \\
5255.955 \\
5307.518 \\
5422.941\end{array}$ & $\begin{array}{l}+0.934 \\
+0.453 \\
-0.980 \\
-0.940 \\
-1.415\end{array}$ & $\begin{array}{l}5166.2 \\
5221.335\end{array}$ & $\begin{array}{l}\text { lab } \\
\text { lab }\end{array}$ \\
\hline 127515.235 & $\left({ }^{3} \mathrm{G}\right) 4 \mathrm{f}$ & $5[7]$ & $13 / 2$ & $\begin{array}{l}105763.270 \\
106045.690 \\
108133.440 \\
108181.550 \\
108387.920 \\
108463.910 \\
108648.695 \\
108775.080 \\
109049.600 \\
109683.280\end{array}$ & $\begin{array}{l}\left({ }^{3} \mathrm{~F}\right) 4 \mathrm{~d}^{2} \mathrm{H}_{11 / 2} \\
\left({ }^{3} \mathrm{H}\right) 4 \mathrm{~d}^{2} \mathrm{H}_{11 / 2} \\
\left({ }^{3} \mathrm{G}\right) 4 \mathrm{~d}^{4} \mathrm{H}_{13 / 2} \\
\left({ }^{3} \mathrm{G}\right) 4 \mathrm{~d}^{4} \mathrm{G}_{11 / 2} \\
\left({ }^{3} \mathrm{G}\right) 4 \mathrm{~d}^{4} \mathrm{H}_{11 / 2} \\
\left({ }^{3} \mathrm{G}\right) 4 \mathrm{~d}^{4} \mathrm{I}_{13 / 2} \\
\left({ }^{1} \mathrm{I}\right) 5 \mathrm{~s} \mathrm{e}^{2} \mathrm{I}_{13 / 2} \\
\left({ }^{3} \mathrm{G}\right) 4 \mathrm{~d}^{4} \mathrm{I}_{11 / 2} \\
\left({ }^{3} \mathrm{G}\right) 4 \mathrm{~d}^{2} \mathrm{I}_{13 / 2} \\
\left({ }^{3} \mathrm{G}\right) 4 \mathrm{~d}^{2} \mathrm{H}_{11 / 2}\end{array}$ & $\begin{array}{l}4595.998 \\
4656.457 \\
5158.044 \\
5170.879 \\
5226.670 \\
5247.518 \\
5298.915 \\
5334.651 \\
5413.960 \\
5606.354\end{array}$ & $\begin{array}{l}-1.059 \\
-0.284 \\
-0.684 \\
-0.639 \\
+0.474 \\
+0.157 \\
-1.299 \\
-0.859 \\
-0.246 \\
+0.514\end{array}$ & $\begin{array}{l}5226.686 \\
5247.536 \\
\\
5606.38\end{array}$ & $\begin{array}{l}\text { lab } \\
\text { lab }\end{array}$ \\
\hline 127489.429 & $\left({ }^{3} \mathrm{G}\right) 4 \mathrm{f}$ & $5[6]$ & $13 / 2$ & $\begin{array}{l}103600.430 \\
106045.690 \\
108133.440 \\
108181.550 \\
108337.860 \\
108387.920 \\
108463.910 \\
108648.695 \\
109683.280\end{array}$ & $\begin{array}{l}\left({ }^{3} \mathrm{H}\right) 4 d^{4} \mathrm{G}_{11 / 2} \\
\left({ }^{3} \mathrm{H}\right) 4 \mathrm{~d}^{2} \mathrm{H}_{11 / 2} \\
\left({ }^{3} \mathrm{G}\right) 4 \mathrm{~d}^{4} \mathrm{H}_{13 / 2} \\
\left({ }^{3} \mathrm{G}\right) 4 \mathrm{~d}^{4} \mathrm{G}_{11 / 2} \\
\left({ }^{3} \mathrm{G}\right) 4 \mathrm{~d}^{4} \mathrm{I}_{15 / 2} \\
\left({ }^{3} \mathrm{G}\right) 4 \mathrm{~d}^{4} \mathrm{H}_{11 / 2} \\
\left({ }^{3} \mathrm{G}\right) 4 \mathrm{~d}^{4} \mathrm{I}_{13 / 2} \\
\left({ }^{1} \mathrm{I}\right) 5 \mathrm{~s} \mathrm{e}^{2} \mathrm{I}_{13 / 2} \\
\left({ }^{3} \mathrm{G}\right) 4 \mathrm{~d}^{2} \mathrm{H}_{11 / 2}\end{array}$ & $\begin{array}{l}4184.848 \\
4662.061 \\
5164.921 \\
5177.791 \\
5220.051 \\
5233.732 \\
5254.636 \\
5306.173 \\
5614.479\end{array}$ & $\begin{array}{l}-1.133 \\
-1.312 \\
+0.601 \\
+0.705 \\
-0.463 \\
-1.225 \\
-0.596 \\
-0.818 \\
-0.728\end{array}$ & $\begin{array}{l}5164.9 \\
5177.77\end{array}$ & $\begin{array}{l}\text { lab } \\
\text { lab }\end{array}$ \\
\hline 127489.977 & $\left({ }^{3} \mathrm{G}\right) 4 \mathrm{f}$ & $5[6]$ & $11 / 2$ & $\begin{array}{l}103600.430 \\
103683.070 \\
106045.690 \\
106722.170 \\
106924.430 \\
108133.440 \\
108181.550 \\
108387.920 \\
108391.500 \\
109049.600 \\
109625.200 \\
109683.280 \\
109811.920 \\
110008.300\end{array}$ & $\begin{array}{l}\left({ }^{3} \mathrm{H}\right) 4 d^{4} \mathrm{G}_{11 / 2} \\
\left({ }^{5} \mathrm{D}\right) 5 \mathrm{~d}^{4} \mathrm{~F}_{9 / 2} \\
\left({ }^{3} \mathrm{H}\right) 4 \mathrm{~d}^{2} \mathrm{H}_{11 / 2} \\
\left({ }^{3} \mathrm{~F}\right) 4 \mathrm{~d}^{4} \mathrm{~F}_{9 / 2} \\
\left({ }^{3} \mathrm{~F}\right) 4 \mathrm{~d}^{2} \mathrm{G}_{9 / 2} \\
\left({ }^{3} \mathrm{G}\right) 4 \mathrm{~d}^{4} \mathrm{H}_{13 / 2} \\
\left({ }^{3} \mathrm{G}\right) 4 \mathrm{~d}^{4} \mathrm{G}_{11 / 2} \\
\left({ }^{3} \mathrm{G}\right) 4 \mathrm{~d}^{4} \mathrm{H}_{11 / 2} \\
\left({ }^{3} \mathrm{G}\right) 4 \mathrm{~d}^{4} \mathrm{G}_{9 / 2} \\
\left({ }^{3} \mathrm{G}\right) 4 \mathrm{~d}^{2} \mathrm{I}_{13 / 2} \\
\left({ }^{3} \mathrm{G}\right) 4 \mathrm{~d}^{2} \mathrm{G}_{9 / 2} \\
\left({ }^{3} \mathrm{G}\right) 4 \mathrm{~d}^{2} \mathrm{H}_{11 / 2} \\
\left({ }^{3} \mathrm{G}\right) 4 \mathrm{~d}^{4} \mathrm{~F}_{9 / 2} \\
\left.{ }^{3} \mathrm{G}\right) 4 \mathrm{~d}^{2} \mathrm{H}_{9 / 2}\end{array}$ & $\begin{array}{l}4184.752 \\
4199.279 \\
4661.942 \\
4813.800 \\
4861.143 \\
5164.775 \\
5177.644 \\
5233.581 \\
5234.562 \\
5421.376 \\
5596.053 \\
5614.306 \\
5655.161 \\
5718.689\end{array}$ & $\begin{array}{l}-1.422 \\
-1.301 \\
-1.108 \\
-0.314 \\
-0.513 \\
-0.273 \\
+0.437 \\
-0.349 \\
-0.887 \\
-1.110 \\
-0.050 \\
-0.230 \\
-0.047 \\
-0.545\end{array}$ & $\begin{array}{l}4813.8 \\
5164.77 \\
5177.64 \\
5233.58 \\
\\
5655.15\end{array}$ & $\begin{array}{l}\text { lab } \\
\text { computed too strong }\end{array}$ \\
\hline 127482.748 & $\left({ }^{3} \mathrm{G}\right) 4 \mathrm{f}$ & $5[5]$ & $11 / 2$ & $\begin{array}{l}105763.270 \\
106045.690 \\
106722.170 \\
108133.440 \\
108181.550 \\
108387.920 \\
108391.500\end{array}$ & $\begin{array}{l}\left({ }^{3} \mathrm{~F}\right) 4 d^{2} \mathrm{H}_{11 / 2} \\
\left({ }^{3} \mathrm{H}\right) 4 \mathrm{~d}^{2} \mathrm{H}_{11 / 2} \\
\left({ }^{3} \mathrm{~F}\right) 4 \mathrm{~d}^{4} \mathrm{~F}_{9 / 2} \\
\left({ }^{3} \mathrm{G}\right) 4 \mathrm{~d}^{4} \mathrm{H}_{13 / 2} \\
\left({ }^{3} \mathrm{G}\right) 4 \mathrm{~d}^{4} \mathrm{G}_{11 / 2} \\
\left({ }^{3} \mathrm{G}\right) 4 \mathrm{~d}^{4} \mathrm{H}_{11 / 2} \\
\left({ }^{3} \mathrm{G}\right) 4 \mathrm{~d}^{4} \mathrm{G}_{9 / 2}\end{array}$ & $\begin{array}{l}4602.873 \\
4663.514 \\
4815.476 \\
5166.704 \\
5179.583 \\
5235.563 \\
5236.545\end{array}$ & $\begin{array}{l}-1.478 \\
-0.736 \\
-0.239 \\
-0.401 \\
+0.320 \\
-0.190 \\
+0.191\end{array}$ & 5235.585 & $\begin{array}{l}\text { computed too strong } \\
\text { computed too strong } \\
\text { blend } \\
\text { blend } \\
\text { blend, computed too strong }\end{array}$ \\
\hline
\end{tabular}


F. Castelli and R. L. Kurucz: New Fe II energy levels from stellar spectra

Table 9. continued.

\begin{tabular}{|c|c|c|c|c|c|c|c|c|c|}
\hline \multicolumn{4}{|c|}{ Upper level } & \multicolumn{2}{|c|}{ Lower level } & \multirow{2}{*}{$\begin{array}{c}\lambda(\text { calc }) \\
\AA \\
\end{array}$} & \multirow{2}{*}{$\begin{array}{c}\log g f \\
\text { KUR }\end{array}$} & \multirow{2}{*}{$\begin{array}{c}\lambda(\mathrm{obs}) \\
\AA\end{array}$} & \multirow[t]{2}{*}{ Notes } \\
\hline $\mathrm{cm}^{-1}$ & & & $J$ & $\mathrm{~cm}^{-1}$ & & & & & \\
\hline 127482.748 & cont. & & & $\begin{array}{l}108463.910 \\
108648.695 \\
108775.080 \\
109625.200 \\
109683.280 \\
109811.920 \\
110008.300\end{array}$ & $\begin{array}{l}\left({ }^{3} \mathrm{G}\right) 4 \mathrm{~d}^{4} \mathrm{I}_{13 / 2} \\
\left({ }^{1} \mathrm{I}\right) 5 \mathrm{~s} \mathrm{e} \mathrm{e}^{2} \mathrm{I}_{13 / 2} \\
\left({ }^{3} \mathrm{G}\right) 4 \mathrm{~d}^{4} \mathrm{I}_{11 / 2} \\
\left({ }^{3} \mathrm{G}\right) 4 \mathrm{~d}^{2} \mathrm{G}_{9 / 2} \\
\left({ }^{3} \mathrm{G}\right) 4 \mathrm{~d}^{2} \mathrm{H}_{11 / 2} \\
\left({ }^{3} \mathrm{G}\right) 4 \mathrm{~d}^{4} \mathrm{~F}_{9 / 2} \\
\left({ }^{3} \mathrm{G}\right) 4 \mathrm{~d}^{2} \mathrm{H}_{9 / 2}\end{array}$ & $\begin{array}{l}5256.482 \\
5308.055 \\
5343.915 \\
5598.319 \\
5616.586 \\
5657.474 \\
5721.054\end{array}$ & $\begin{array}{l}-0.830 \\
-1.341 \\
-1.043 \\
-0.100 \\
-0.042 \\
-0.662 \\
-0.506\end{array}$ & $\begin{array}{l}5598.32 \\
5616.6 \\
5657.50\end{array}$ & $\begin{array}{l}\text { computed too weak } \\
\text { computed too weak } \\
\text { computed too weak }\end{array}$ \\
\hline 127485.362 & $\left({ }^{3} \mathrm{G}\right) 4 \mathrm{f}$ & $5[4]$ & $9 / 2$ & $\begin{array}{l}104107.950 \\
104481.590 \\
105775.491 \\
106045.690 \\
106722.170 \\
106767.210 \\
106924.430 \\
108181.550 \\
108365.320 \\
108387.920 \\
108391.500 \\
108537.610 \\
108577.560 \\
109625.200 \\
109683.280 \\
109811.920 \\
109901.500 \\
110008.300 \\
110167.280 \\
110570.300\end{array}$ & 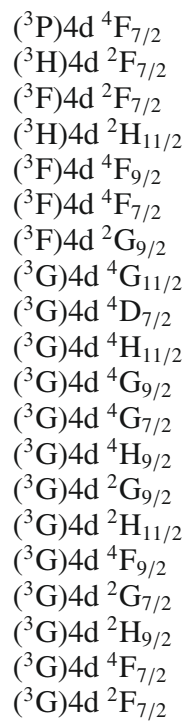 & $\begin{array}{l}4276.430 \\
4345.891 \\
4604.910 \\
4662.945 \\
4814.870 \\
4825.337 \\
4862.235 \\
5178.882 \\
5228.658 \\
5234.846 \\
5235.828 \\
5276.203 \\
5287.351 \\
5597.499 \\
5615.762 \\
5656.638 \\
5685.455 \\
5720.199 \\
5772.711 \\
5910.253\end{array}$ & $\begin{array}{l}-1.168 \\
-1.316 \\
-1.176 \\
-1.404 \\
-0.945 \\
-1.318 \\
-0.425 \\
-0.635 \\
-0.224 \\
-0.695 \\
-0.195 \\
-1.169 \\
-1.391 \\
+0.251 \\
-0.466 \\
-0.349 \\
-0.333 \\
-0.468 \\
-1.064 \\
-0.120\end{array}$ & $\begin{array}{l}5234.80 \\
5235.80 \\
\\
5597.50 \\
5615.75 \\
5656.55 \\
5685.45 \\
5720.20\end{array}$ & $\begin{array}{l}\text { blend } \\
\text { blend } \\
\text { computed too strong } \\
\text { blend } \\
\text { blend } \mathrm{H} 2 \mathrm{O}\end{array}$ \\
\hline 127485.699 & $\left({ }^{3} \mathrm{G}\right) 4 \mathrm{f}$ & $5[4]$ & $7 / 2$ & $\begin{array}{l}103683.070 \\
106722.170 \\
106767.210 \\
106900.370 \\
106924.430 \\
108365.320 \\
108391.500 \\
108537.610 \\
109625.200 \\
109811.920 \\
110065.750 \\
110167.280 \\
110570.300\end{array}$ & $\begin{array}{l}\left({ }^{5} \mathrm{D}\right) 5 \mathrm{~d}^{4} \mathrm{~F}_{9 / 2} \\
\left({ }^{3} \mathrm{~F}\right) 4 \mathrm{~d}^{4} \mathrm{~F}_{9 / 2} \\
\left({ }^{3} \mathrm{~F}\right) 4 \mathrm{~d}^{4} \mathrm{~F}_{7 / 2} \\
\left({ }^{3} \mathrm{~F}\right) 4 \mathrm{~d}^{2} \mathrm{G}_{7 / 2} \\
\left({ }^{3} \mathrm{~F}\right) 4 \mathrm{~d}^{2} \mathrm{G}_{9 / 2} \\
\left({ }^{3} \mathrm{G}\right) 4 d^{4} \mathrm{D}_{7 / 2} \\
\left({ }^{3} \mathrm{G}\right) 4 d^{4} \mathrm{G}_{9 / 2} \\
\left({ }^{3} \mathrm{G}\right) 4 d^{4} \mathrm{G}_{7 / 2} \\
\left({ }^{3} \mathrm{G}\right) 4 \mathrm{~d}^{2} \mathrm{G}_{9 / 2} \\
\left({ }^{3} \mathrm{G}\right) 4 \mathrm{~d}^{4} \mathrm{~F}_{9 / 2} \\
\left({ }^{3} \mathrm{G}\right) 4 d^{2} \mathrm{D}_{5 / 2} \\
\left({ }^{3} \mathrm{G}\right) 4 d^{4} \mathrm{~F}_{7 / 2} \\
\left({ }^{3} \mathrm{G}\right) 4 d^{2} \mathrm{~F}_{7 / 2}\end{array}$ & $\begin{array}{l}4200.033 \\
4814.791 \\
4825.259 \\
4856.472 \\
4862.155 \\
5228.566 \\
5235.735 \\
5276.109 \\
5597.394 \\
5656.530 \\
5738.953 \\
5772.598 \\
5910.135\end{array}$ & $\begin{array}{l}-1.226 \\
+0.017 \\
-0.375 \\
-1.384 \\
-0.753 \\
+0.266 \\
-0.618 \\
-0.999 \\
-1.025 \\
+0.034 \\
-1.494 \\
-0.676 \\
-1.369\end{array}$ & $\begin{array}{l}4814.8 \\
4825.30\end{array}$ & $\begin{array}{l}\text { computed too strong } \\
\text { blend } \\
\text { blend } \\
\text { blend }\end{array}$ \\
\hline 127510.913 & $\left({ }^{3} \mathrm{G}\right) 4 \mathrm{f}$ & $5[3]$ & $5 / 2$ & $\begin{array}{l}106767.210 \\
106900.370 \\
108365.320 \\
108537.610 \\
110065.750 \\
110167.280 \\
110570.300\end{array}$ & $\begin{array}{l}\left({ }^{3} \mathrm{~F}\right) 4 \mathrm{~d}^{4} \mathrm{~F}_{7 / 2} \\
\left({ }^{3} \mathrm{~F}\right) 4 \mathrm{~d}^{2} \mathrm{G}_{7 / 2} \\
\left({ }^{3} \mathrm{G}\right) 4 \mathrm{~d}^{4} \mathrm{D}_{7 / 2} \\
\left({ }^{3} \mathrm{G}\right) 4 \mathrm{~d}^{4} \mathrm{G}_{7 / 2} \\
\left({ }^{3} \mathrm{G}\right) 4 \mathrm{~d}^{2} \mathrm{D}_{5 / 2} \\
\left({ }^{3} \mathrm{G}\right) 4 \mathrm{~d}^{4} \mathrm{~F}_{7 / 2} \\
\left({ }^{3} \mathrm{G}\right) 4 \mathrm{~d}^{2} \mathrm{~F}_{7 / 2}\end{array}$ & $\begin{array}{l}4819.393 \\
4850.531 \\
5221.680 \\
5269.097 \\
5730.658 \\
5764.206 \\
5901.339\end{array}$ & $\begin{array}{l}-0.294 \\
-1.345 \\
+0.447 \\
-0.794 \\
-0.761 \\
-0.654 \\
-1.193\end{array}$ & $\begin{array}{l}4819.40 \\
5221.68 \\
5369.12\end{array}$ & blend \\
\hline 127487.681 & $\left({ }^{3} \mathrm{G}\right) 4 \mathrm{f}$ & $5[2]$ & $3 / 2$ & $\begin{array}{l}106866.760 \\
108642.410 \\
110065.750 \\
\end{array}$ & $\begin{array}{l}\left({ }^{3} F\right) 4 d^{4} F_{5 / 2} \\
\left({ }^{3} G\right) 4 d{ }^{4} D_{5 / 2} \\
\left({ }^{3} G\right) 4 d{ }^{2} D_{5 / 2}\end{array}$ & $\begin{array}{l}4848.090 \\
5304.895 \\
5738.300 \\
\end{array}$ & $\begin{array}{l}-0.945 \\
-0.425 \\
-0.104 \\
\end{array}$ & $\begin{array}{r}5304.89 \\
5738.30 \\
\end{array}$ & blend \\
\hline
\end{tabular}


Table 9. continued.

\begin{tabular}{|c|c|c|c|c|c|c|c|c|c|}
\hline \multicolumn{4}{|c|}{ Upper level } & \multicolumn{2}{|c|}{ Lower level } & \multirow{2}{*}{$\begin{array}{c}\lambda(\text { calc }) \\
\AA\end{array}$} & \multirow{2}{*}{$\begin{array}{l}\log g f \\
\text { KUR }\end{array}$} & \multirow{2}{*}{$\begin{array}{c}\lambda(\text { obs }) \\
\AA \\
\end{array}$} & \multirow[t]{2}{*}{ Notes } \\
\hline $\mathrm{cm}^{-1}$ & & & $J$ & $\mathrm{~cm}^{-1}$ & & & & & \\
\hline 127892.981 & $\left({ }^{3} \mathrm{G}\right) 4 \mathrm{f}$ & $4[7]$ & $15 / 2$ & $\begin{array}{l}104064.670 \\
104622.300 \\
108133.440 \\
108463.910 \\
108648.695 \\
109049.600\end{array}$ & $\begin{array}{l}\left({ }^{3} \mathrm{H}\right) 4 \mathrm{~d}^{4} \mathrm{I}_{13 / 2} \\
\left({ }^{3} \mathrm{H}\right) 4 \mathrm{~d}^{2} \mathrm{I}_{13 / 2} \\
\left({ }^{3} \mathrm{G}\right) 4 \mathrm{~d}^{4} \mathrm{H}_{13 / 2} \\
\left({ }^{3} \mathrm{G}\right) 4 \mathrm{~d}^{4} \mathrm{I}_{13 / 2} \\
\left({ }^{1} \mathrm{I}\right) 5 \mathrm{~s} \mathrm{e} \mathrm{e}^{2} \mathrm{I}_{13 / 2} \\
\left({ }^{3} \mathrm{G}\right) 4 \mathrm{~d}^{2} \mathrm{I}_{13 / 2}\end{array}$ & $\begin{array}{l}4195.506 \\
4296.044 \\
5059.436 \\
5145.493 \\
5194.901 \\
5305.427\end{array}$ & $\begin{array}{l}-1.455 \\
-1.387 \\
-0.484 \\
-0.007 \\
+0.482 \\
+0.862\end{array}$ & $\begin{array}{l}5059.42 \\
5145.5 \\
5305.42\end{array}$ & $\begin{array}{l}\text { lab } \\
\text { blend } \\
\text { lab }\end{array}$ \\
\hline 127895.260 & $\left({ }^{3} \mathrm{G}\right) 4 \mathrm{f}$ & $4[7]$ & $13 / 2$ & $\begin{array}{l}104174.270 \\
108387.920 \\
108630.429 \\
108648.695 \\
108775.080 \\
109389.880\end{array}$ & $\begin{array}{l}\left({ }^{3} \mathrm{H}\right) 4 \mathrm{~d}^{4} \mathrm{I}_{11 / 2} \\
\left({ }^{3} \mathrm{G}\right) 4 \mathrm{~d}^{4} \mathrm{H}_{11 / 2} \\
\left({ }^{1} \mathrm{I}\right) 5 \mathrm{~s} \mathrm{e} \mathrm{e}^{2} \mathrm{I}_{11 / 2} \\
\left({ }^{1} \mathrm{I}\right) 5 \mathrm{~s} \mathrm{e} \mathrm{e}^{2} \mathrm{I}_{13 / 2} \\
\left({ }^{3} \mathrm{G}\right) 4 \mathrm{~d}^{4} \mathrm{I}_{11 / 2} \\
\left({ }^{3} \mathrm{G}\right) 4 \mathrm{~d}^{2} \mathrm{I}_{11 / 2}\end{array}$ & $\begin{array}{l}4214.489 \\
5124.848 \\
5189.361 \\
5194.286 \\
5228.621 \\
5402.332\end{array}$ & $\begin{array}{l}-1.351 \\
-0.679 \\
-0.144 \\
-1.434 \\
+0.896 \\
+0.099\end{array}$ & $\begin{array}{l}5189.371 \\
5228.635 \\
5402.32\end{array}$ & $\begin{array}{l}\text { blend } \\
\text { lab } \\
\text { lab } \\
\text { lab }\end{array}$ \\
\hline 127875.000 & $\left({ }^{3} \mathrm{G}\right) 4 \mathrm{f}$ & $4[6]$ & $13 / 2$ & $\begin{array}{l}106045.690 \\
108133.440 \\
108387.920 \\
108463.910 \\
108648.695 \\
109049.600 \\
109683.280\end{array}$ & $\begin{array}{l}\left({ }^{3} \mathrm{H}\right) 4 \mathrm{~d}^{2} \mathrm{H}_{11 / 2} \\
\left({ }^{3} \mathrm{G}\right) 4 \mathrm{~d}^{4} \mathrm{H}_{13 / 2} \\
\left({ }^{3} \mathrm{G}\right) 4 \mathrm{~d}^{4} \mathrm{H}_{11 / 2} \\
\left({ }^{3} \mathrm{G}\right) 4 \mathrm{~d}^{4} \mathrm{I}_{13 / 2} \\
\left({ }^{1} \mathrm{I}\right) 5 \mathrm{~s} \mathrm{e}^{2} \mathrm{I}_{13 / 2} \\
\left({ }^{3} \mathrm{G}\right) 4 \mathrm{~d}^{2} \mathrm{I}_{13 / 2} \\
\left({ }^{3} \mathrm{G}\right) 4 \mathrm{~d}^{2} \mathrm{H}_{11 / 2}\end{array}$ & $\begin{array}{l}4579.713 \\
5064.044 \\
5130.176 \\
5150.259 \\
5199.759 \\
5310.495 \\
5495.480\end{array}$ & $\begin{array}{l}-0.754 \\
-1.045 \\
+0.662 \\
-0.700 \\
-0.190 \\
+0.113 \\
+0.481\end{array}$ & $\begin{array}{l}5130.18 \\
\\
5310.5 \\
5495.49\end{array}$ & $\begin{array}{l}\text { lab } \\
\text { blend } \\
\text { lab } \\
\text { lab, J78 }\end{array}$ \\
\hline 127880.436 & $\left({ }^{3} \mathrm{G}\right) 4 \mathrm{f}$ & $4[6]$ & $11 / 2$ & $\begin{array}{l}106097.520 \\
108387.920 \\
108391.500 \\
108577.560 \\
108630.429 \\
108775.080 \\
108929.040 \\
109389.880 \\
109625.200 \\
109683.280 \\
109811.920 \\
110008.300\end{array}$ & $\begin{array}{l}\left({ }^{3} \mathrm{H}\right) 4 d^{2} \mathrm{H}_{9 / 2} \\
\left({ }^{3} \mathrm{G}\right) 4 d^{4} \mathrm{H}_{11 / 2} \\
\left({ }^{3} \mathrm{G}\right) 4 \mathrm{~d}^{4} \mathrm{G}_{9 / 2} \\
\left({ }^{3} \mathrm{G}\right) 4 d^{4} \mathrm{H}_{9 / 2} \\
\left({ }^{1} \mathrm{I}\right) 5 \mathrm{~s} \mathrm{e} \mathrm{e}^{2} \mathrm{I}_{11 / 2} \\
\left({ }^{3} \mathrm{G}\right) 4 \mathrm{~d}^{4} \mathrm{I}_{11 / 2} \\
\left({ }^{3} \mathrm{G}\right) 4 \mathrm{~d}^{4} \mathrm{I}_{9 / 2} \\
\left({ }^{3} \mathrm{G}\right) 4 \mathrm{~d}^{2} \mathrm{I}_{11 / 2} \\
\left({ }^{3} \mathrm{G}\right) 4 \mathrm{~d}^{2} \mathrm{G}_{9 / 2} \\
\left({ }^{3} \mathrm{G}\right) 4 \mathrm{~d}^{2} \mathrm{H}_{11 / 2} \\
\left({ }^{3} \mathrm{G}\right) 4 \mathrm{~d}^{4} \mathrm{~F}_{9 / 2} \\
\left({ }^{3} \mathrm{G}\right) 4 \mathrm{~d}^{2} \mathrm{H}_{9 / 2}\end{array}$ & $\begin{array}{l}4589.468 \\
5128.745 \\
5129.687 \\
5179.133 \\
5193.357 \\
5232.678 \\
5275.188 \\
5406.663 \\
5476.359 \\
5493.838 \\
5532.952 \\
5593.749\end{array}$ & $\begin{array}{l}-0.765 \\
-0.375 \\
-1.085 \\
+0.652 \\
-0.797 \\
-0.047 \\
-0.897 \\
-0.491 \\
-0.333 \\
-1.052 \\
-0.700 \\
+0.039\end{array}$ & $\begin{array}{l}5179.14 \\
5476.38 \\
5593.85\end{array}$ & $\begin{array}{l}\text { blend } \\
\text { lab } \\
\text { blend }\end{array}$ \\
\hline 127869.158 & $\left({ }^{3} \mathrm{G}\right) 4 \mathrm{f}$ & $4[5]$ & $11 / 2$ & $\begin{array}{l}106045.690 \\
106722.170 \\
108387.920 \\
108391.500 \\
108577.560 \\
108648.695 \\
108775.080 \\
108929.040 \\
109049.600 \\
109625.200 \\
109683.280 \\
109811.920 \\
110008.300\end{array}$ & $\begin{array}{l}\left({ }^{3} \mathrm{H}\right) 4 \mathrm{~d}^{2} \mathrm{H}_{11 / 2} \\
\left({ }^{3} \mathrm{~F}\right) 4 \mathrm{~d}^{4} \mathrm{~F}_{9 / 2} \\
\left({ }^{3} \mathrm{G}\right) 4 \mathrm{~d}^{4} \mathrm{H}_{11 / 2} \\
\left({ }^{3} \mathrm{G}\right) 4 \mathrm{~d}^{4} \mathrm{G}_{9 / 2} \\
\left({ }^{3} \mathrm{G}\right) 4 \mathrm{~d}^{4} \mathrm{H}_{9 / 2} \\
\left({ }^{1} \mathrm{I}\right) 5 \mathrm{~s} \mathrm{e}^{2} \mathrm{I}_{13 / 2} \\
\left({ }^{3} \mathrm{G}\right) 4 \mathrm{~d}^{4} \mathrm{I}_{11 / 2} \\
\left({ }^{3} \mathrm{G}\right) 4 \mathrm{~d}^{4} \mathrm{I}_{9 / 2} \\
\left({ }^{3} \mathrm{G}\right) 4 \mathrm{~d}^{2} \mathrm{I}_{13 / 2} \\
\left({ }^{3} \mathrm{G}\right) 4 \mathrm{~d}^{2} \mathrm{G}_{9 / 2} \\
\left({ }^{3} \mathrm{G}\right) 4 \mathrm{~d}^{2} \mathrm{H}_{11 / 2} \\
\left({ }^{3} \mathrm{G}\right) 4 \mathrm{~d}^{4} \mathrm{~F}_{9 / 2} \\
\left.{ }^{3} \mathrm{G}\right) 4 \mathrm{~d}^{2} \mathrm{H}_{9 / 2}\end{array}$ & $\begin{array}{l}4580.939 \\
4727.483 \\
5131.714 \\
5132.657 \\
5182.161 \\
5201.340 \\
5235.768 \\
5278.329 \\
5312.143 \\
5479.744 \\
5497.245 \\
5536.408 \\
5597.281\end{array}$ & $\begin{array}{l}-1.153 \\
-0.893 \\
+0.220 \\
+0.408 \\
-0.938 \\
-1.171 \\
-0.234 \\
-1.413 \\
-0.846 \\
-0.089 \\
+0.050 \\
-0.555 \\
-0.105\end{array}$ & $\begin{array}{l}5479.72 \\
5497.25 \\
5536.40 \\
5597.30\end{array}$ & $\begin{array}{l}\text { lab } \\
\text { blend } \\
\text { blend }\end{array}$ \\
\hline 127855.952 & $\left({ }^{3} \mathrm{G}\right) 4 \mathrm{f}$ & $4[5]$ & $9 / 2$ & $\begin{array}{l}106722.170 \\
106767.210 \\
106900.370 \\
108365.320 \\
108387.920 \\
108391.500 \\
108577.560 \\
108709.450 \\
109625.200 \\
\end{array}$ & $\begin{array}{l}\left({ }^{3} \mathrm{~F}\right) 4 d^{4} \mathrm{~F}_{9 / 2} \\
\left({ }^{3} \mathrm{~F}\right) 4 \mathrm{~d}^{4} \mathrm{~F}_{7 / 2} \\
\left({ }^{3} \mathrm{~F}\right) 4 \mathrm{~d}^{2} \mathrm{G}_{7 / 2} \\
\left({ }^{3} \mathrm{G}\right) 4 \mathrm{~d}^{4} \mathrm{D}_{7 / 2} \\
\left({ }^{3} \mathrm{G}\right) 4 \mathrm{~d}^{4} \mathrm{H}_{11 / 2} \\
\left({ }^{3} \mathrm{G}\right) 4 d^{4} \mathrm{G}_{9 / 2} \\
\left({ }^{3} \mathrm{G}\right) 4 d^{4} \mathrm{H}_{9 / 2} \\
\left({ }^{3} \mathrm{G}\right) 4 d^{4} \mathrm{H}_{7 / 2} \\
\left({ }^{3} \mathrm{G}\right) 4 d^{2} \mathrm{G}_{9 / 2}\end{array}$ & $\begin{array}{l}4730.437 \\
4740.541 \\
4770.664 \\
5129.241 \\
5135.195 \\
5136.140 \\
5185.710 \\
5221.432 \\
5483.714 \\
\end{array}$ & $\begin{array}{l}-0.906 \\
-0.409 \\
-1.118 \\
-0.301 \\
-0.409 \\
+0.294 \\
-0.829 \\
-1.407 \\
+0.010 \\
\end{array}$ & 5129.25 & $\begin{array}{l}\text { blend } \\
\text { blend }\end{array}$ \\
\hline
\end{tabular}


F. Castelli and R. L. Kurucz: New Fe II energy levels from stellar spectra

Table 9. continued.

\begin{tabular}{|c|c|c|c|c|c|c|c|c|c|}
\hline \multicolumn{4}{|c|}{ Upper level } & \multicolumn{2}{|c|}{ Lower level } & \multirow{2}{*}{$\begin{array}{c}\lambda(\text { calc }) \\
\AA \\
\AA\end{array}$} & \multirow{2}{*}{$\begin{array}{c}\log g f \\
\text { KUR }\end{array}$} & \multirow{2}{*}{$\begin{array}{c}\lambda(\mathrm{obs}) \\
\AA \\
\end{array}$} & \multirow[t]{2}{*}{ Notes } \\
\hline $\mathrm{cm}^{-1}$ & & & $J$ & $\mathrm{~cm}^{-1}$ & & & & & \\
\hline 127855.952 & cont. & & & $\begin{array}{l}109683.280 \\
109811.920 \\
109901.500 \\
110167.280 \\
110570.300\end{array}$ & $\begin{array}{l}\left({ }^{3} \mathrm{G}\right) 4 d^{2} \mathrm{H}_{11 / 2} \\
\left({ }^{3} \mathrm{G}\right) 4 \mathrm{~d}^{4} \mathrm{~F}_{9 / 2} \\
\left({ }^{3} \mathrm{G}\right) 4 \mathrm{~d}^{2} \mathrm{G}_{7 / 2} \\
\left({ }^{3} \mathrm{G}\right) 4 d^{4} \mathrm{~F}_{7 / 2} \\
\left({ }^{3} \mathrm{G}\right) 4 \mathrm{~d}^{2} \mathrm{~F}_{7 / 2}\end{array}$ & $\begin{array}{l}5501.240 \\
5540.460 \\
5568.103 \\
5651.767 \\
5783.541\end{array}$ & $\begin{array}{l}-0.659 \\
-0.431 \\
-0.216 \\
-0.160 \\
-0.854\end{array}$ & $\begin{array}{l}5540.47 \\
5568.10 \\
5651.78\end{array}$ & computed too weak \\
\hline 127869.892 & $\left({ }^{3} \mathrm{G}\right) 4 \mathrm{f}$ & $4[4]$ & $9 / 2$ & $\begin{array}{l}106097.520 \\
106900.370 \\
108365.320 \\
108391.500 \\
108537.610 \\
108577.560 \\
108709.450 \\
108775.080 \\
108929.040 \\
109389.880 \\
109901.500 \\
110008.300 \\
110167.280 \\
110570.300\end{array}$ & $\begin{array}{l}\left({ }^{3} \mathrm{H}\right) 4 d^{2} \mathrm{H}_{9 / 2} \\
\left({ }^{3} \mathrm{~F}\right) 4 d^{2} \mathrm{G}_{7 / 2} \\
\left({ }^{3} \mathrm{G}\right) 4 d^{4} \mathrm{D}_{7 / 2} \\
\left({ }^{3} \mathrm{G}\right) 4 d^{4} \mathrm{G}_{9 / 2} \\
\left({ }^{3} \mathrm{G}\right) 4 d^{4} \mathrm{G}_{7 / 2} \\
\left({ }^{3} \mathrm{G}\right) 4 d^{4} \mathrm{H}_{9 / 2} \\
\left({ }^{3} \mathrm{G}\right) 4 d^{4} \mathrm{H}_{7 / 2} \\
\left({ }^{3} \mathrm{G}\right) 4 d^{4} \mathrm{I}_{11 / 2} \\
\left({ }^{3} \mathrm{G}\right) 4 d^{4} \mathrm{I}_{9 / 2} \\
\left({ }^{3} \mathrm{G}\right) 4 d^{2} \mathrm{I}_{11 / 2} \\
\left({ }^{3} \mathrm{G}\right) 4 d^{2} \mathrm{G}_{7 / 2} \\
\left({ }^{3} \mathrm{G}\right) 4 d^{2} \mathrm{H}_{9 / 2} \\
\left({ }^{3} \mathrm{G}\right) 4 d^{4} \mathrm{~F}_{7 / 2} \\
\left({ }^{3} \mathrm{G}\right) 4 d^{2} \mathrm{~F}_{7 / 2}\end{array}$ & $\begin{array}{l}4591.690 \\
4767.493 \\
5125.575 \\
5132.464 \\
5171.255 \\
5181.963 \\
5217.634 \\
5235.567 \\
5278.125 \\
5409.748 \\
5563.783 \\
5597.051 \\
5647.317 \\
5778.881\end{array}$ & $\begin{array}{l}-1.043 \\
-1.141 \\
-1.117 \\
-0.690 \\
+0.332 \\
+0.101 \\
-1.196 \\
-0.810 \\
-0.704 \\
-1.407 \\
-0.269 \\
+0.023 \\
-0.723 \\
-0.074\end{array}$ & $\begin{array}{l}5171.25 \\
5181.97 \\
\\
5563.79 \\
5597.05 \\
5778.88\end{array}$ & $\begin{array}{l}\text { no soectrum } \\
\text { no spectrum } \\
\text { weak } \\
\text { blend } \\
\text { lab, J78 } \\
\text { lab } \\
\text { weak } \\
\text { blend } \\
\text { blend } \\
\text { blend }\end{array}$ \\
\hline 127874.745 & $\left({ }^{3} \mathrm{G}\right) 4 \mathrm{f}$ & $4[3]$ & $5 / 2$ & $\begin{array}{l}106767.210 \\
106796.660 \\
106866.760 \\
107407.800 \\
108365.320 \\
108537.610 \\
108613.960 \\
108642.410 \\
108859.470 \\
109901.500 \\
110065.750 \\
110167.280 \\
110428.280 \\
110570.300 \\
110611.800\end{array}$ & $\begin{array}{l}\left({ }^{3} \mathrm{~F}\right) 4 d^{4} \mathrm{~F}_{7 / 2} \\
\left({ }^{3} \mathrm{~F}\right) 4 d^{4} \mathrm{P}_{5 / 2} \\
\left({ }^{3} \mathrm{~F}\right) 4 \mathrm{~d}^{4} \mathrm{~F}_{5 / 2} \\
\left({ }^{3} \mathrm{~F}\right) 4 \mathrm{~d}^{2} \mathrm{D}_{5 / 2} \\
\left({ }^{3} \mathrm{G}\right) 4 \mathrm{~d}^{4} \mathrm{D}_{7 / 2} \\
\left({ }^{3} \mathrm{G}\right) 4 \mathrm{~d}^{4} \mathrm{G}_{7 / 2} \\
\left({ }^{3} \mathrm{G}\right) 4 \mathrm{~d}^{4} \mathrm{G}_{5 / 2} \\
\left({ }^{3} \mathrm{G}\right) 4 \mathrm{~d}^{4} \mathrm{D}_{5 / 2} \\
\left({ }^{3} \mathrm{G}\right) 4 \mathrm{~d}^{4} \mathrm{D}_{3 / 2} \\
\left({ }^{3} \mathrm{G}\right) 4 \mathrm{~d}^{2} \mathrm{G}_{7 / 2} \\
\left({ }^{3} \mathrm{G}\right) 4 \mathrm{~d}^{2} \mathrm{D}_{5 / 2} \\
\left({ }^{3} \mathrm{G}\right) 4 \mathrm{~d}^{4} \mathrm{~F}_{7 / 2} \\
\left({ }^{3} \mathrm{G}\right) 4 \mathrm{~d}^{4} \mathrm{~F}_{5 / 2} \\
\left({ }^{3} \mathrm{G}\right) 4 d^{2} \mathrm{~F}_{7 / 2} \\
\left({ }^{3} \mathrm{G}\right) 4 \mathrm{~d}^{2} \mathrm{~F}_{5 / 2}\end{array}$ & $\begin{array}{l}4736.320 \\
4742.937 \\
4758.764 \\
4884.563 \\
5124.300 \\
5169.957 \\
5190.451 \\
5198.129 \\
5257.467 \\
5562.281 \\
5613.582 \\
5645.769 \\
5730.231 \\
5777.260 \\
5791.149\end{array}$ & $\begin{array}{l}-0.862 \\
-1.442 \\
-0.354 \\
-1.137 \\
-0.351 \\
-0.493 \\
-1.336 \\
-0.577 \\
-1.074 \\
-0.790 \\
-0.302 \\
-0.897 \\
-0.236 \\
-0.288 \\
-1.493\end{array}$ & $\begin{array}{l}5124.3 \\
5169.95 \\
5198.12\end{array}$ & $\begin{array}{l}\text { blend } \\
\text { weak } \\
\text { weak } \\
\text { blend } \\
\text { weak } \\
\text { blend } \\
\text { computed too weak } \\
\text { blend }\end{array}$ \\
\hline 128110.214 & $\left({ }^{3} \mathrm{G}\right) 4 \mathrm{f}$ & $3[6]$ & $13 / 2$ & $\begin{array}{l}104765.450 \\
108387.920 \\
108630.429 \\
108775.080 \\
109389.880\end{array}$ & $\begin{array}{l}\left({ }^{3} \mathrm{H}\right) 4 \mathrm{~d}^{2} \mathrm{I}_{11 / 2} \\
\left({ }^{3} \mathrm{G}\right) 4 \mathrm{~d}^{4} \mathrm{H}_{11 / 2} \\
\left({ }^{1} \mathrm{I}\right) 5 \mathrm{~s} \mathrm{e} \mathrm{e}^{2} \mathrm{I}_{11 / 2} \\
\left({ }^{3} \mathrm{G}\right) 4 \mathrm{~d}^{4} \mathrm{I}_{11 / 2} \\
\left({ }^{3} \mathrm{G}\right) 4 \mathrm{~d}^{2} \mathrm{I}_{11 / 2}\end{array}$ & $\begin{array}{l}4282.411 \\
5068.991 \\
5132.097 \\
5170.492 \\
5340.300\end{array}$ & $\begin{array}{l}-1.266 \\
-0.821 \\
-0.929 \\
+0.154 \\
+0.922\end{array}$ & $\begin{array}{l}5068.99 \\
5170.5 \\
5340.30\end{array}$ & $\begin{array}{l}\text { blend } \\
\text { blend } \\
\text { lab } \\
\text { lab, J78 }\end{array}$ \\
\hline 128071.171 & $\left({ }^{3} \mathrm{~F}\right) 4 \mathrm{f}$ & $3[5]$ & $11 / 2$ & $\begin{array}{l}106097.520 \\
106924.430 \\
108387.920 \\
108391.500 \\
108577.560 \\
108775.080 \\
108929.040 \\
109389.880 \\
106925.200 \\
110008.300\end{array}$ & $\begin{array}{l}\left({ }^{3} \mathrm{H}\right) 4 \mathrm{~d}^{2} \mathrm{H}_{9 / 2} \\
\left({ }^{3} \mathrm{~F}\right) 4 \mathrm{~d}^{2} \mathrm{G}_{9 / 2} \\
\left({ }^{3} \mathrm{G}\right) 4 \mathrm{~d}^{4} \mathrm{H}_{11 / 2} \\
\left({ }^{3} \mathrm{G}\right) 4 \mathrm{~d}^{4} \mathrm{G}_{9 / 2} \\
\left({ }^{3} \mathrm{G}\right) 4 \mathrm{~d}^{4} \mathrm{H}_{9 / 2} \\
\left({ }^{3} \mathrm{G}\right) 4 \mathrm{~d}^{4} \mathrm{I}_{11 / 2} \\
\left({ }^{3} \mathrm{G}\right) 4 \mathrm{~d}^{4} \mathrm{I}_{9 / 2} \\
\left({ }^{3} \mathrm{G}\right) 4 \mathrm{~d}^{2} \mathrm{I}_{11 / 2} \\
\left({ }^{3} \mathrm{G}\right) 4 \mathrm{~d}^{2} \mathrm{G}_{9 / 2} \\
\left({ }^{3} \mathrm{G}\right) 4 \mathrm{~d}^{2} \mathrm{H}_{9 / 2}\end{array}$ & $\begin{array}{l}4549.630 \\
4727.539 \\
5079.046 \\
5079.970 \\
5128.457 \\
5180.954 \\
5222.625 \\
5351.461 \\
5419.731 \\
5534.681\end{array}$ & $\begin{array}{l}-0.731 \\
-0.926 \\
-1.376 \\
-1.401 \\
+0.377 \\
-0.687 \\
-0.245 \\
+0.043 \\
-0.013 \\
+0.459\end{array}$ & $\begin{array}{l}5128.47 \\
5222.62 \\
5351.47 \\
5419.73 \\
5534.68\end{array}$ & $\begin{array}{l}\text { no spectrum } \\
\text { no spectrum } \\
\text { blend } \\
\text { at the continuum level } \\
\text { lab } \\
\text { blend } \\
\text { computed too strong } \\
\text { lab }\end{array}$ \\
\hline
\end{tabular}


Table 9. continued.

\begin{tabular}{|c|c|c|c|c|c|c|c|c|c|}
\hline \multicolumn{4}{|c|}{ Upper level } & \multicolumn{2}{|c|}{ Lower level } & \multirow{2}{*}{$\begin{array}{c}\lambda(\mathrm{calc}) \\
\AA\end{array}$} & \multirow{2}{*}{$\begin{array}{c}\log g f \\
\text { KUR }\end{array}$} & \multirow{2}{*}{$\begin{array}{c}\lambda(\text { obs }) \\
\AA\end{array}$} & \multirow[t]{2}{*}{ Notes } \\
\hline $\mathrm{cm}^{-1}$ & & & $J$ & $\mathrm{~cm}^{-1}$ & & & & & \\
\hline 128055.658 & $\left({ }^{3} \mathrm{~F}\right) 4 \mathrm{f}$ & $3[5]$ & $9 / 2$ & $\begin{array}{l}106097.520 \\
106767.210 \\
106924.430 \\
108537.610 \\
108577.560 \\
108709.450 \\
108775.080 \\
109389.880 \\
106925.200 \\
109901.500 \\
110008.300 \\
110167.280 \\
110570.300\end{array}$ & $\begin{array}{l}\left({ }^{3} \mathrm{H}\right) 4 d^{2} \mathrm{H}_{9 / 2} \\
\left({ }^{3} \mathrm{~F}\right) 4 \mathrm{~d}^{4} \mathrm{~F}_{7 / 2} \\
\left({ }^{3} \mathrm{~F}\right) 4 \mathrm{~d}^{2} \mathrm{G}_{9 / 2} \\
\left({ }^{3} \mathrm{G}\right) 4 \mathrm{~d}^{4} \mathrm{G}_{7 / 2} \\
\left({ }^{3} \mathrm{G}\right) 4 \mathrm{~d}^{4} \mathrm{H}_{9 / 2} \\
\left({ }^{3} \mathrm{G}\right) 4 \mathrm{~d}^{4} \mathrm{H}_{7 / 2} \\
\left({ }^{3} \mathrm{G}\right) 4 \mathrm{~d}^{4} \mathrm{I}_{11 / 2} \\
\left({ }^{3} \mathrm{G}\right) 4 \mathrm{~d}^{2} \mathrm{I}_{11 / 2} \\
\left({ }^{3} \mathrm{G}\right) 4 \mathrm{~d}^{2} \mathrm{G}_{9 / 2} \\
\left({ }^{3} \mathrm{G}\right) 4 \mathrm{~d}^{2} \mathrm{G}_{7 / 2} \\
\left({ }^{3} \mathrm{G}\right) 4 \mathrm{~d}^{2} \mathrm{H}_{9 / 2} \\
\left({ }^{3} \mathrm{G}\right) 4 \mathrm{~d}^{4} \mathrm{~F}_{7 / 2} \\
\left({ }^{3} \mathrm{G}\right) 4 \mathrm{~d}^{2} \mathrm{~F}_{7 / 2}\end{array}$ & $\begin{array}{l}4552.844 \\
4696.069 \\
4731.009 \\
5122.036 \\
5132.541 \\
5167.532 \\
5185.122 \\
5355.908 \\
5424.293 \\
5506.850 \\
5539.439 \\
5588.670 \\
5717.485\end{array}$ & $\begin{array}{l}-1.204 \\
-0.812 \\
-1.380 \\
+0.148 \\
+0.038 \\
-0.521 \\
-1.448 \\
-0.925 \\
-0.649 \\
+0.159 \\
+0.045 \\
-0.697 \\
-0.176\end{array}$ & $\begin{array}{l}5122.02 \\
5132.55 \\
5185.141 \\
5355.9 \\
\\
5506.85 \\
5539.41 \\
5588.65 \\
5717.50\end{array}$ & $\begin{array}{l}\text { no spectrum } \\
\text { no spectrum } \\
\text { no spectrum } \\
\text { lab } \\
\text { lab } \\
\text { blend } \\
\text { blend } \\
\text { weak } \\
\text { blend }\end{array}$ \\
\hline 128062.710 & $\left({ }^{3} \mathrm{~F}\right) 4 \mathrm{f}$ & $3[4]$ & $9 / 2$ & $\begin{array}{l}106900.370 \\
108709.450 \\
108929.040 \\
109901.500 \\
110008.300 \\
110570.300\end{array}$ & $\begin{array}{l}\left({ }^{3} \mathrm{~F}\right) 4 \mathrm{~d}^{2} \mathrm{G}_{7 / 2} \\
\left({ }^{3} \mathrm{G}\right) 4 \mathrm{~d}^{4} \mathrm{H}_{7 / 2} \\
\left({ }^{3} \mathrm{G}\right) 4 \mathrm{~d}^{4} \mathrm{I}_{9 / 2} \\
\left({ }^{3} \mathrm{G}\right) 4 \mathrm{~d}^{2} \mathrm{G}_{7 / 2} \\
\left({ }^{3} \mathrm{G}\right) 4 \mathrm{~d}^{2} \mathrm{H}_{9 / 2} \\
\left({ }^{3} \mathrm{G}\right) 4 \mathrm{~d}^{2} \mathrm{~F}_{7 / 2}\end{array}$ & $\begin{array}{l}4724.054 \\
5165.649 \\
5224.934 \\
5504.712 \\
5537.275 \\
5715.180\end{array}$ & $\begin{array}{l}-1.276 \\
+0.734 \\
+0.139 \\
-0.840 \\
-1.268 \\
-1.173\end{array}$ & $\begin{array}{l}5165.65 \\
5224.938\end{array}$ & $\begin{array}{l}\text { no spectrum } \\
\text { lab } \\
\text { not observed } \\
\text { at the level of the noise } \\
\text { at the level of the noise }\end{array}$ \\
\hline 128066.823 & $\left({ }^{3} \mathrm{~F}\right) 4 \mathrm{f}$ & $3[4]$ & $7 / 2$ & $\begin{array}{l}104023.910 \\
106208.560 \\
106767.210 \\
106900.370 \\
108537.610 \\
108577.560 \\
108613.960 \\
108709.450 \\
108929.040 \\
109901.500 \\
110008.300 \\
110570.300 \\
110611.800\end{array}$ & $\begin{array}{l}\left({ }^{3} \mathrm{H}\right) 4 d^{4} \mathrm{G}_{5 / 2} \\
\left({ }^{3} \mathrm{~F}\right) 4 \mathrm{~d}^{2} \mathrm{~F}_{5 / 2} \\
\left({ }^{3} \mathrm{~F}\right) 4 \mathrm{~d}^{4} \mathrm{~F}_{7 / 2} \\
\left({ }^{3} \mathrm{~F}\right) 4 \mathrm{~d}^{2} \mathrm{G}_{7 / 2} \\
\left({ }^{3} \mathrm{G}\right) 4 \mathrm{~d}^{4} \mathrm{G}_{7 / 2} \\
\left({ }^{3} \mathrm{G}\right) 4 \mathrm{~d}^{4} \mathrm{H}_{9 / 2} \\
\left({ }^{3} \mathrm{G}\right) 4 \mathrm{~d}^{4} \mathrm{G}_{5 / 2} \\
\left({ }^{3} \mathrm{G}\right) 4 \mathrm{~d}^{4} \mathrm{H}_{7 / 2} \\
\left({ }^{3} \mathrm{G}\right) 4 \mathrm{~d}^{4} \mathrm{I}_{9 / 2} \\
\left({ }^{3} \mathrm{G}\right) 4 \mathrm{~d}^{2} \mathrm{G}_{7 / 2} \\
\left({ }^{3} \mathrm{G}\right) 4 \mathrm{~d}^{2} \mathrm{H}_{9 / 2} \\
\left({ }^{3} \mathrm{G}\right) 4 \mathrm{~d}^{2} \mathrm{~F}_{7 / 2} \\
\left({ }^{3} \mathrm{G}\right) 4 \mathrm{~d}^{2} \mathrm{~F}_{5 / 2}\end{array}$ & $\begin{array}{l}4158.057 \\
4573.647 \\
4693.607 \\
4723.136 \\
5119.108 \\
5129.601 \\
5139.200 \\
5164.552 \\
5223.811 \\
5503.465 \\
5536.014 \\
5713.836 \\
5727.421\end{array}$ & $\begin{array}{l}-1.351 \\
-1.130 \\
-1.067 \\
-1.319 \\
-0.444 \\
-1.316 \\
+0.196 \\
-0.146 \\
-0.993 \\
-0.078 \\
-0.751 \\
-0.308 \\
-0.043\end{array}$ & $\begin{array}{l}5139.20 \\
5164.52 \\
\\
5536.0 \\
5713.8 \\
5727.45\end{array}$ & $\begin{array}{l}\text { not observed, wrong } \\
\text { no spectrum } \\
\text { no spectrum } \\
\text { no spectrum } \\
\text { computed too strong } \\
\text { blend } \\
\text { lab } \\
\text { computed too weak } \\
\text { blend } \\
\text { blend }\end{array}$ \\
\hline 128063.103 & $\left({ }^{3} \mathrm{G}\right) 4 \mathrm{f}$ & $3[3]$ & $5 / 2$ & $\begin{array}{l}106864.650 \\
106866.760 \\
107430.250 \\
108613.960 \\
108642.410 \\
108709.450 \\
108859.470 \\
109901.500 \\
110428.280 \\
110461.260 \\
110609.540 \\
110611.800\end{array}$ & $\begin{array}{l}\left({ }^{3} G\right) 4 d^{4} F_{3 / 2} \\
\left({ }^{3} F\right) 4 d^{4} F_{5 / 2} \\
\left({ }^{3} F\right) 4 d^{2} D_{3 / 2} \\
\left({ }^{3} G\right) 4 d^{4} G_{5 / 2} \\
\left({ }^{3} G\right) 4 d^{4} D_{5 / 2} \\
\left({ }^{3} G\right) 4 d^{4} H_{7 / 2} \\
\left({ }^{3} G\right) 4 d^{4} D_{3 / 2} \\
\left({ }^{3} G\right) 4 d^{2} G_{5 / 2} \\
\left({ }^{3} G\right) 4 d^{4} F_{5 / 2} \\
\left({ }^{3} G\right) 4 d^{2} D_{3 / 2} \\
\left({ }^{3} G\right) 4 d^{4} F_{3 / 2} \\
\left({ }^{3} G\right) 4 d^{2} F_{5 / 2}\end{array}$ & $\begin{array}{l}4712.005 \\
4716.475 \\
4845.286 \\
5140.183 \\
5147.713 \\
5165.544 \\
5205.898 \\
5504.593 \\
5669.025 \\
5679.647 \\
5727.900 \\
5728.642\end{array}$ & $\begin{array}{l}-0.481 \\
-1.431 \\
-0.946 \\
+0.037 \\
-0.412 \\
-0.693 \\
-0.225 \\
-1.414 \\
-0.651 \\
-1.133 \\
-0.186 \\
-0.772\end{array}$ & $\begin{array}{l}5140.19 \\
5147.71 \\
5205.879 \\
5669.03 \\
5727.90\end{array}$ & $\begin{array}{l}\text { no spectrum } \\
\text { no spectrum } \\
\text { blend,computed too strong } \\
\text { computed too weak } \\
\text { blend } \\
\text { at the continuum level } \\
\text { at the level of the noise } \\
\text { weak }\end{array}$ \\
\hline 128089.313 & $\left({ }^{3} \mathrm{G}\right) 4 \mathrm{f}$ & $3[2]$ & $5 / 2$ & $\begin{array}{l}106208.560 \\
106747.210 \\
106796.660 \\
106866.760 \\
108537.610 \\
108642.410 \\
108859.470 \\
109901.500 \\
110428.280 \\
110461.260 \\
110570.300 \\
110611.800\end{array}$ & $\begin{array}{l}\left({ }^{3} \mathrm{~F}\right) 4 \mathrm{~d}^{2} \mathrm{~F}_{5 / 2} \\
\left({ }^{5} \mathrm{D}\right) 5 \mathrm{~d}^{4} \mathrm{~F}_{7 / 2} \\
\left({ }^{3} \mathrm{~F}\right) 4 \mathrm{~d}^{4} \mathrm{P}_{5 / 2} \\
\left({ }^{3} \mathrm{~F}\right) 4 \mathrm{~d}^{4} \mathrm{~F}_{5 / 2} \\
\left({ }^{3} \mathrm{G}\right) 4 \mathrm{~d}^{4} \mathrm{G}_{7 / 2} \\
\left({ }^{3} \mathrm{G}\right) 4 \mathrm{~d}^{4} \mathrm{D}_{5 / 2} \\
\left({ }^{3} \mathrm{G}\right) 4 \mathrm{~d}^{4} \mathrm{D}_{3 / 2} \\
\left({ }^{3} \mathrm{G}\right) 4 \mathrm{~d}^{2} \mathrm{G}_{5 / 2} \\
\left({ }^{3} \mathrm{G}\right) 4 \mathrm{~d}^{4} \mathrm{~F}_{5 / 2} \\
\left({ }^{3} \mathrm{G}\right) 4 \mathrm{~d}^{2} \mathrm{D}_{3 / 2} \\
\left({ }^{3} \mathrm{G}\right) 4 \mathrm{~d}^{2} \mathrm{~F}_{7 / 2} \\
\left({ }^{3} \mathrm{G}\right) 4 \mathrm{~d}^{2} \mathrm{~F}_{5 / 2}\end{array}$ & $\begin{array}{l}4568.946 \\
4688.657 \\
4695.142 \\
4710.650 \\
5113.219 \\
5140.775 \\
5198.803 \\
5496.660 \\
5660.612 \\
5671.202 \\
5706.501 \\
5720.051\end{array}$ & $\begin{array}{l}-1.396 \\
-1.457 \\
-1.393 \\
-1.102 \\
-1.022 \\
-0.580 \\
-0.577 \\
-0.747 \\
-0.985 \\
-0.429 \\
-0.913 \\
+0.065\end{array}$ & $\begin{array}{l}5671.20 \\
5720.05\end{array}$ & $\begin{array}{l}\text { no spectrum } \\
\text { no spectrum } \\
\text { no spectrum } \\
\text { no spectrum } \\
\text { at the continuum level } \\
\text { blend } \\
\text { blend } \\
\text { blend } \\
\text { blend } \\
\text { at the level of the noise }\end{array}$ \\
\hline
\end{tabular}

